University of Louisville

ThinkIR: The University of Louisville's Institutional Repository

Electronic Theses and Dissertations

$5-2018$

\title{
The far green country.
}

Lincoln A. Sandham

University of Louisville

Follow this and additional works at: https://ir.library.louisville.edu/etd

Part of the Composition Commons

\section{Recommended Citation}

Sandham, Lincoln A., "The far green country." (2018). Electronic Theses and Dissertations. Paper 2916. https://doi.org/10.18297/etd/2916

This Master's Thesis is brought to you for free and open access by ThinkIR: The University of Louisville's Institutional Repository. It has been accepted for inclusion in Electronic Theses and Dissertations by an authorized administrator of ThinkIR: The University of Louisville's Institutional Repository. This title appears here courtesy of the author, who has retained all other copyrights. For more information, please contact thinkir@louisville.edu. 


\title{
The Far Green Country
}

For orchestra

By

Lincoln Sandham

B.M., Baldwin Wallace University, 2016

\begin{abstract}
A Thesis
Submitted to the Faculty of the

School of Music of the University of Louisville

In Partial Fulfillment of the Requirements

For the Degree of
\end{abstract}

Master of Music in Music Composition

School of Music

University of Louisville

Louisville, Kentucky

May 2018 
Copyright (C) 2018 by Lincoln Sandham.

All Rights Reserved. 


\title{
The Far Green Country
}

\author{
For Orchestra
}

\author{
By \\ Lincoln Sandham \\ B.M., Baldwin Wallace University, 2016
}

A Thesis Approved on

May 2, 2018

By the following Thesis Committee:

Steve Rouse

Thesis Director

Marc Satterwhite

Kimcherie Lloyd 


\section{DEDICATION}

This thesis is dedicated to my wife and family who have supported and encouraged me every step of the way. 


\section{ACKNOWLEDGEMENTS}

Dr. Steve Rouse for his invaluable advice and experience, his kind, supportive, and thoughtful guidance, and for countless hours dedicated to making both this Thesis and my time at UofL a successful and rewarding experience. His professionalism and dedication to being the best composer and teacher he can be are priceless models, and have caused me to grow as a composer, musician, and person throughout my time as his student.

Thanks to my committee members, Dr. Marc Satterwhite and Kimcherie Lloyd for their critique, support, and enthusiasm to help.

Thanks to Dr. Kent Hatteburg for his inspiring leadership, and for countless unforgettable experiences performing in his ensembles.

Thanks to Drs. Mark Yeary and Kryzstof Wolek for widening my musical horizons in their classes.

Thanks to Dr. Rebecca Jemian for her leadership, friendship, and encouragement. She has made my first teaching experience extremely rewarding.

Thanks to Chris Fitzgerald for embracing me as a colleague, and for the trust he placed in me as a teaching assistant.

Thanks to Jack Ashworth for sharing his extraordinarily vast body of knowledge with me, and for enthusiastically supporting my interest in early music.

Thanks to Drs. Caroline Ehman and Devin Burke for their model of thoughtful scholarship.

Finally, thanks to my wife and family, for their unending love, support, and encouragement. 
TABLE OF CONTENTS

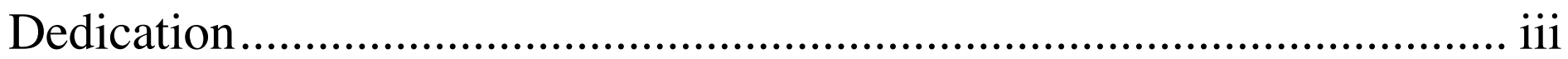

Acknowledgements ................................................................................

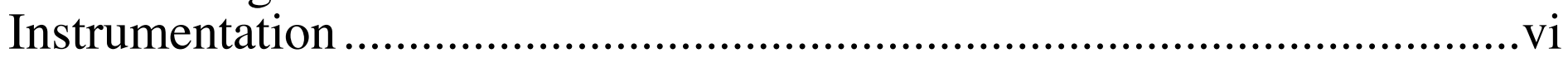

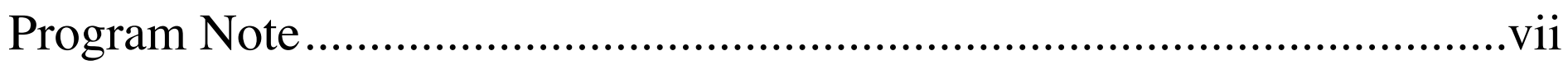

The Far Green Country

I. The Gray Raincurtain ...................................................... 1

II. Silver Glass ................................................................... 22

III. The Far Green Country .................................................. 22

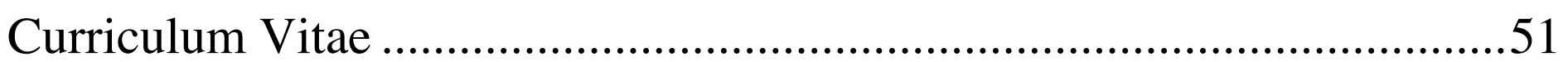




\section{INSTRUMENTATION}

Transposed Score

3 Flutes ( $3^{\text {rd }}$ double Piccolo)

3 Oboes

3 Clarinets in $\mathrm{Bb}$

2 Bassoons

4 Horns in $\mathrm{F}$

3 Trumpets in $\mathrm{C}$

3 Trombones

Tuba

Timpani

Percussion 1

Marimba (range A2-Bb5*), Suspended Cymbal, Vibraphone (may be shared, also bowed), Glockenspiel

Percussion 2

Vibraphone (may be shared, also bowed), Tam-tam, Suspended Cymbal

Percussion 3

Bass Drum, Crotales (range C4-C6* , sounding 15ma, bowed), Suspended Cymbal

Harp

Piano

Violin 1

Violin 2

Viola

Cello

Double Bass

Duration: ca. 17 minutes

Movement 1 - 5 minutes 30 seconds

Movement 2-4 minutes 40 seconds

Movement $3-7$ minutes 15 seconds

*if middle $\mathrm{C}=\mathrm{C} 4$ 


\section{PROGRAM NOTE}

This work was inspired by a beautiful image from J.R.R. Tolkien's The Lord of the Rings:

"And then it seemed to him that as in his dream in the house of Bombadil, the grey rain-curtain turned all to silver glass and was rolled back, and he beheld white shores and beyond them a far green country under a swift sunrise."

$\sim$ The Return of The King, Book VI chapter 9

This imagery inspired the three movements of this work. The first, The Gray Raincurtain, depicts a violent end, the world crashing down around you. The musical material of this movement gradually unravels - the piece is built in several waves, each becoming more and more tumultuous and disturbed, until the final crest shatters and leaves a deafening silence in its wake.

The second movement, Silver Glass, is an in-between place - a transformation from one state of being to another. The sound of silver glass is created with bowed percussion instruments, the rest of the orchestra gradually adding their voices to create a glistening halo of sound.

The final movement, The Far Green Country, emerges from this halo and embodies the excitement and joy of arriving home after a long absence. This movement rotates through key centers, slowly adding accidentals to the orchestral texture, creating the feeling of running ever upward and upward, each step of the way more euphoric than the last. 
Transposed Score
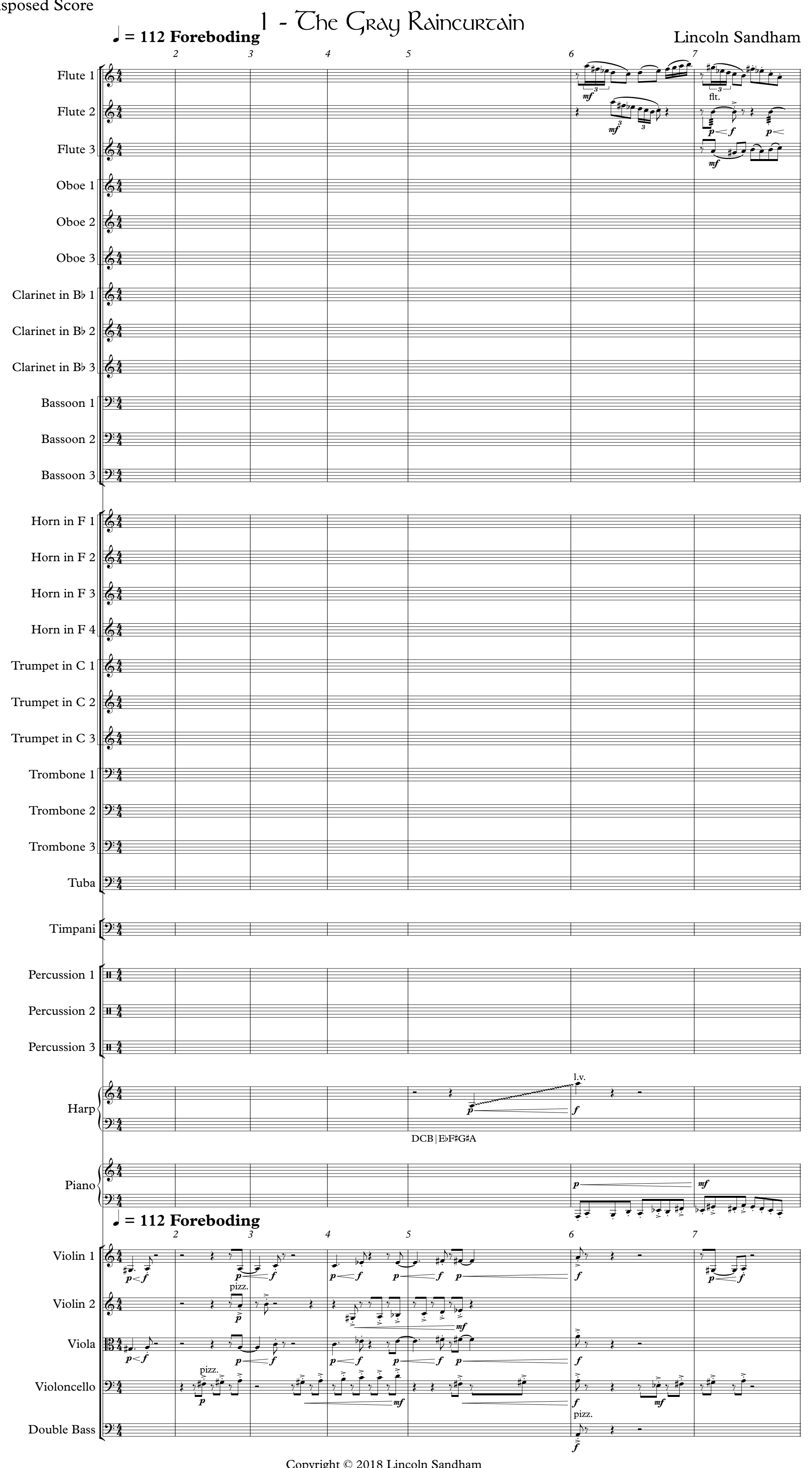

Copyright $(2018$ Lincoln Sandham 

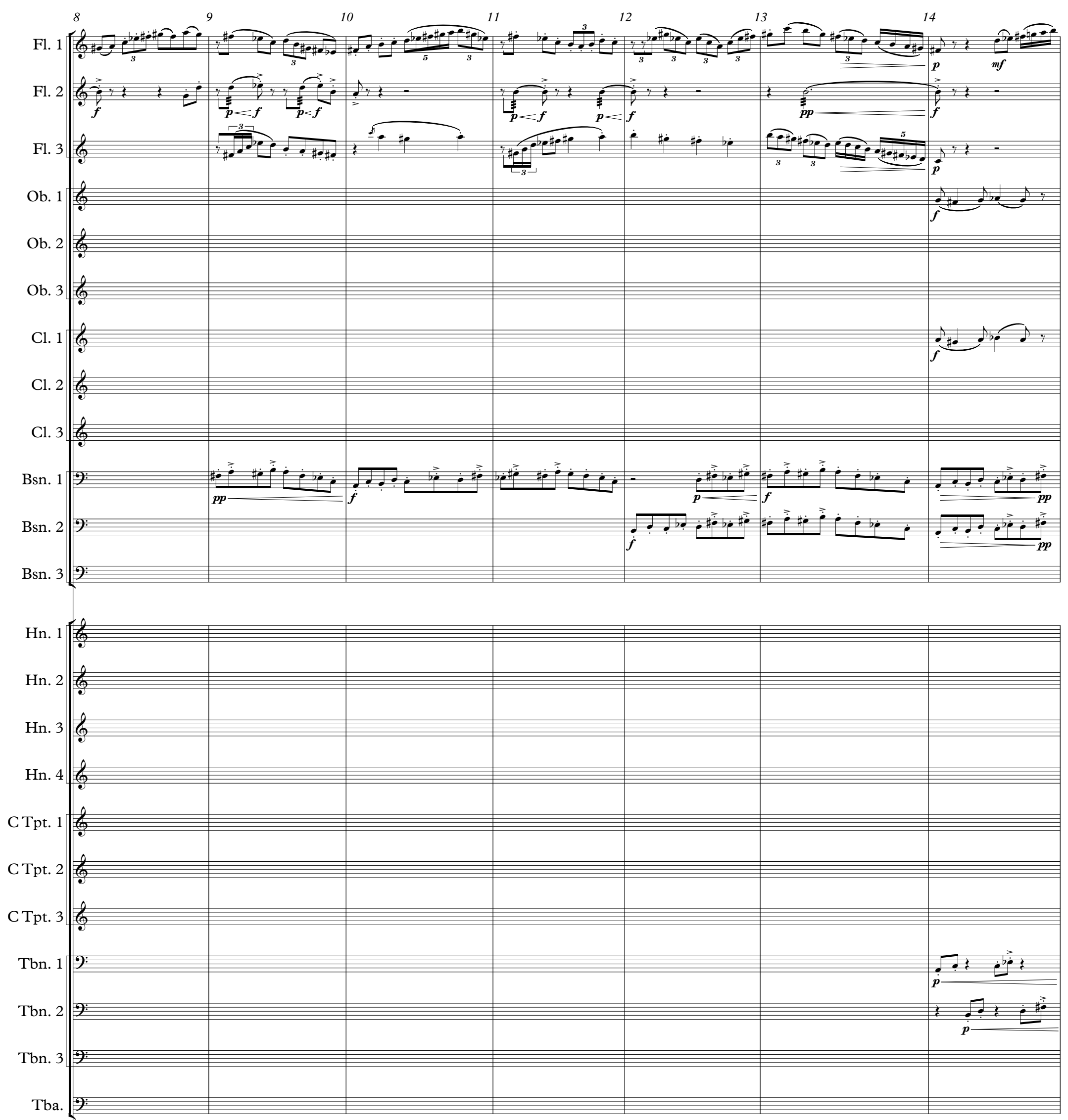

Timp.

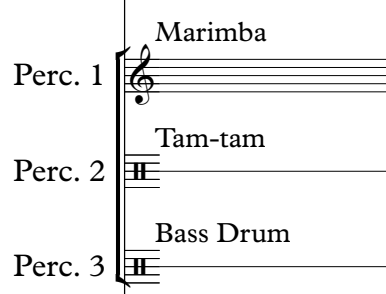
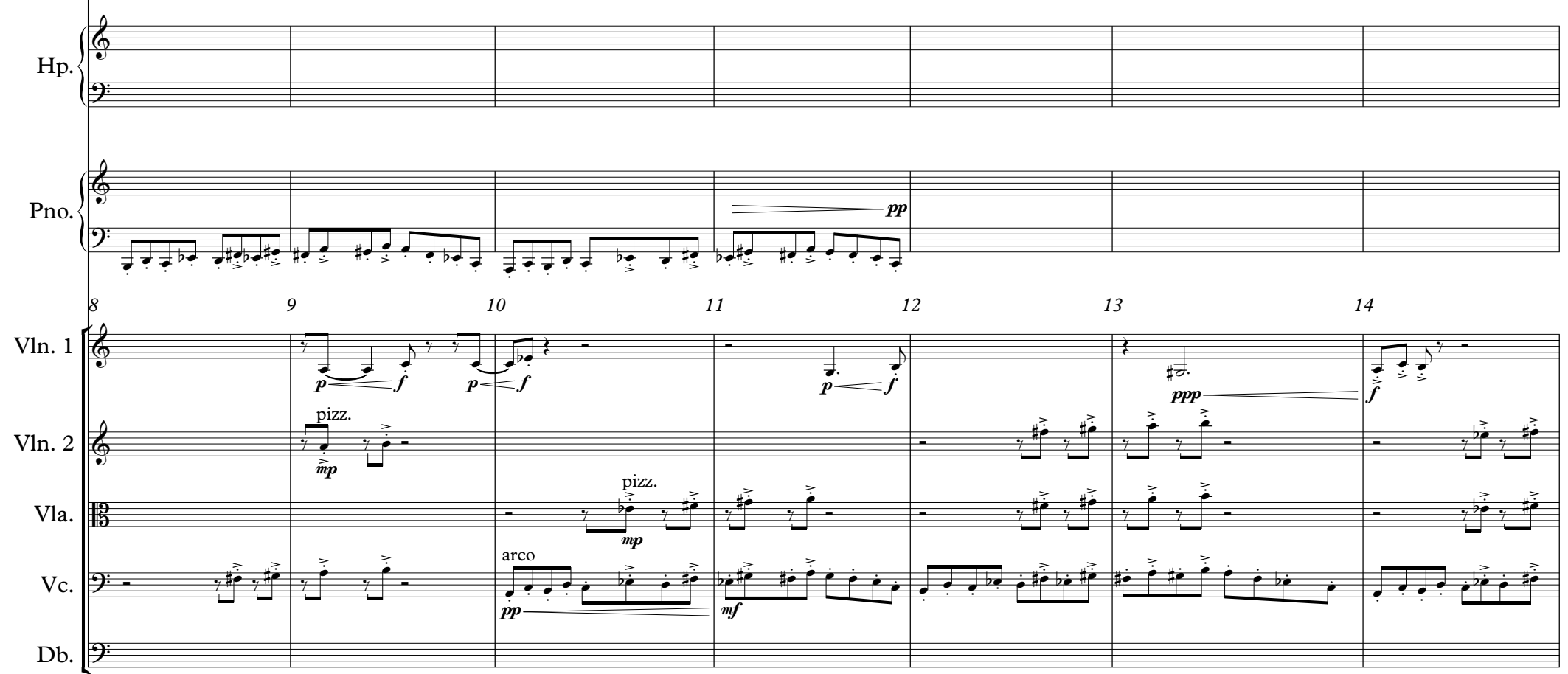

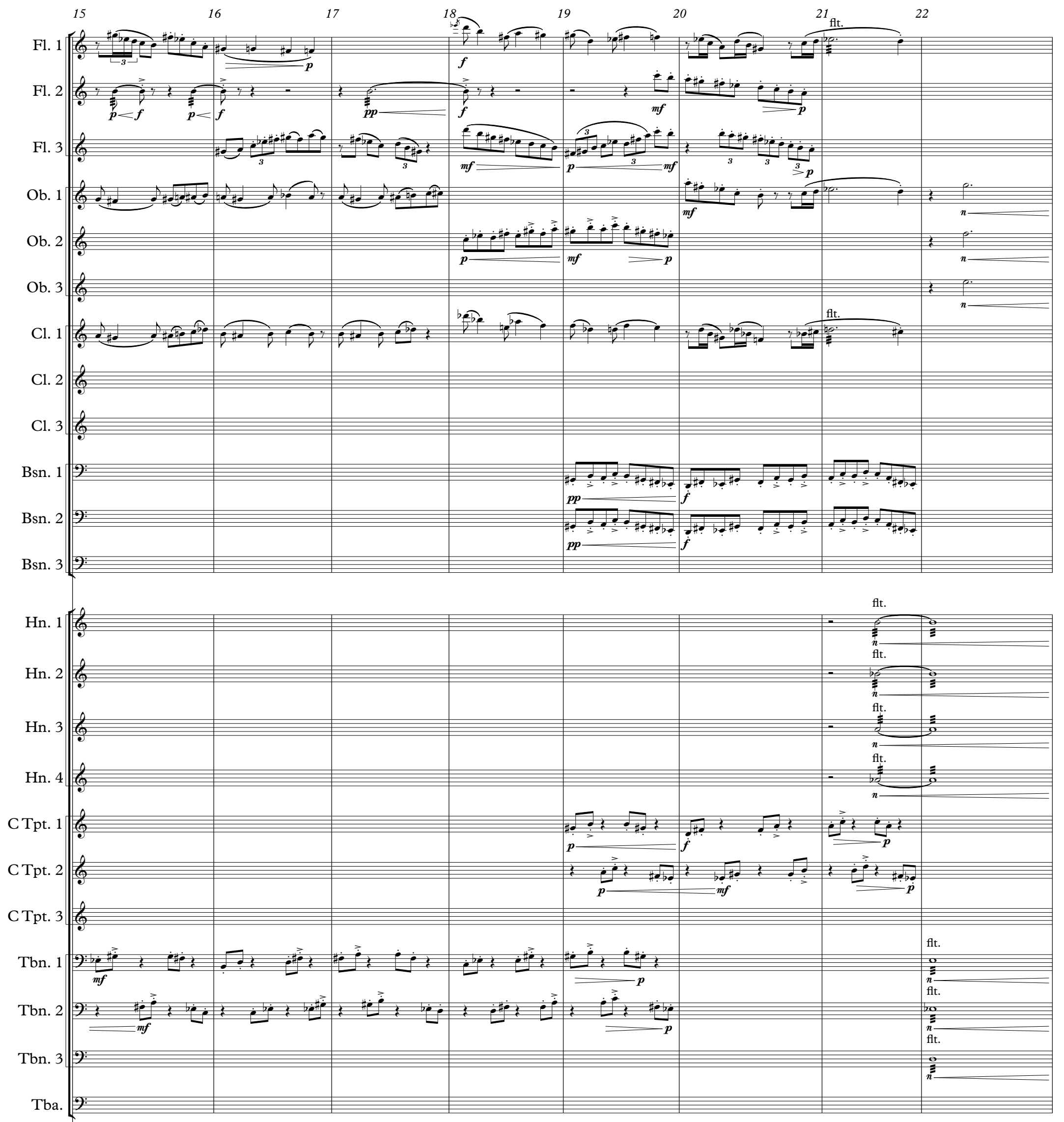
Timp.
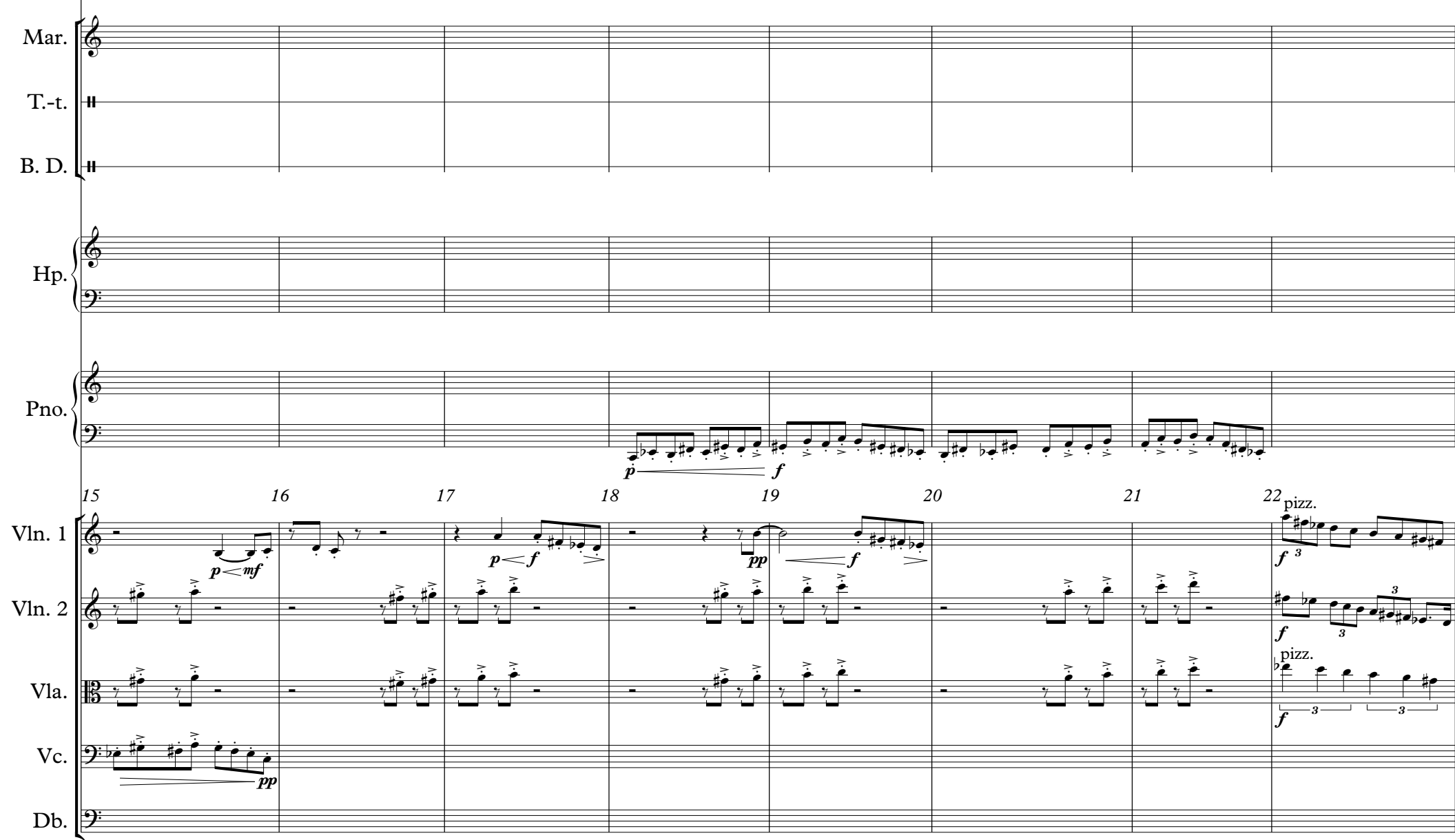

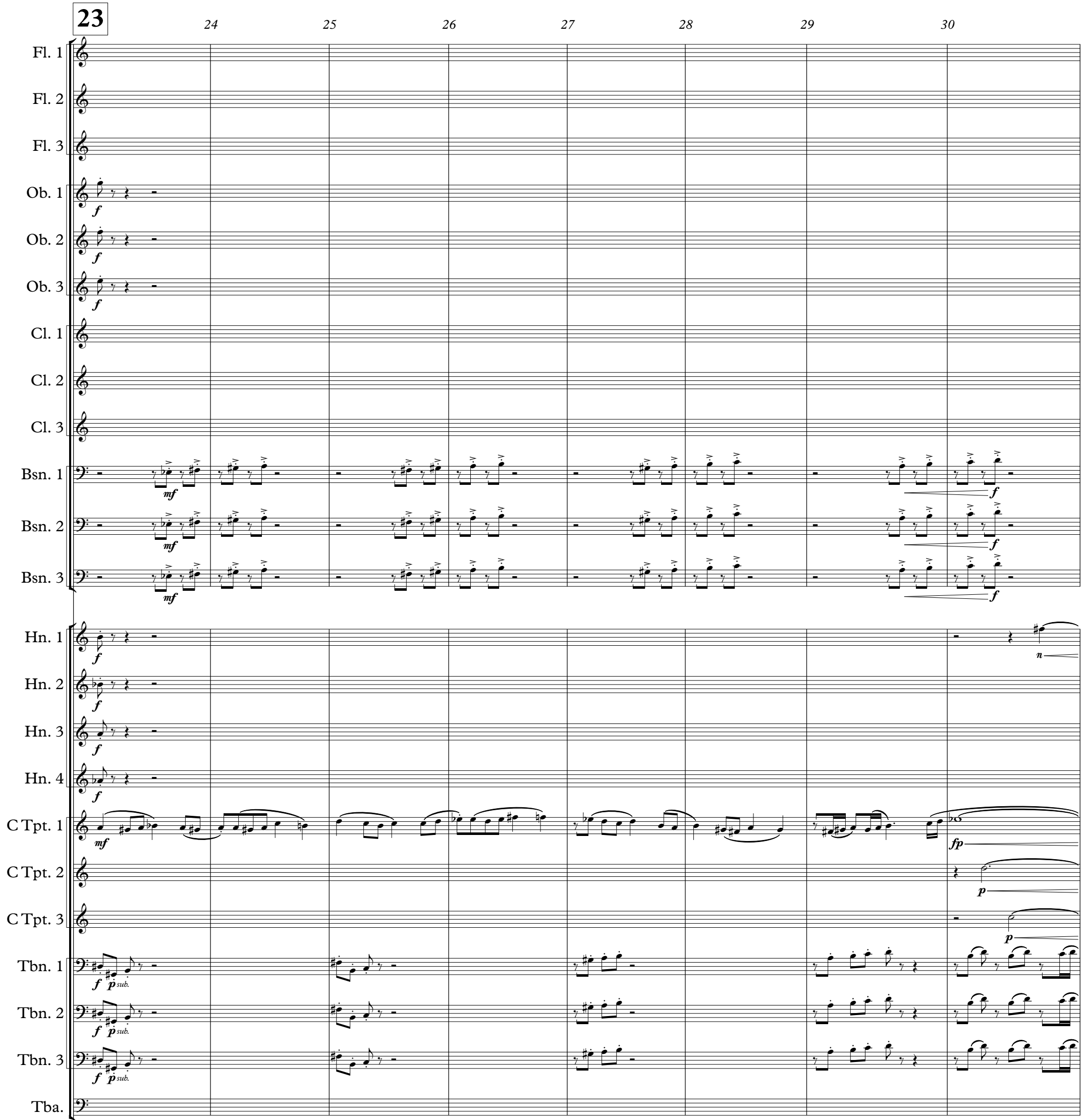

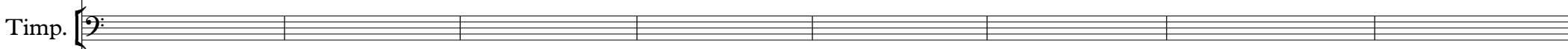
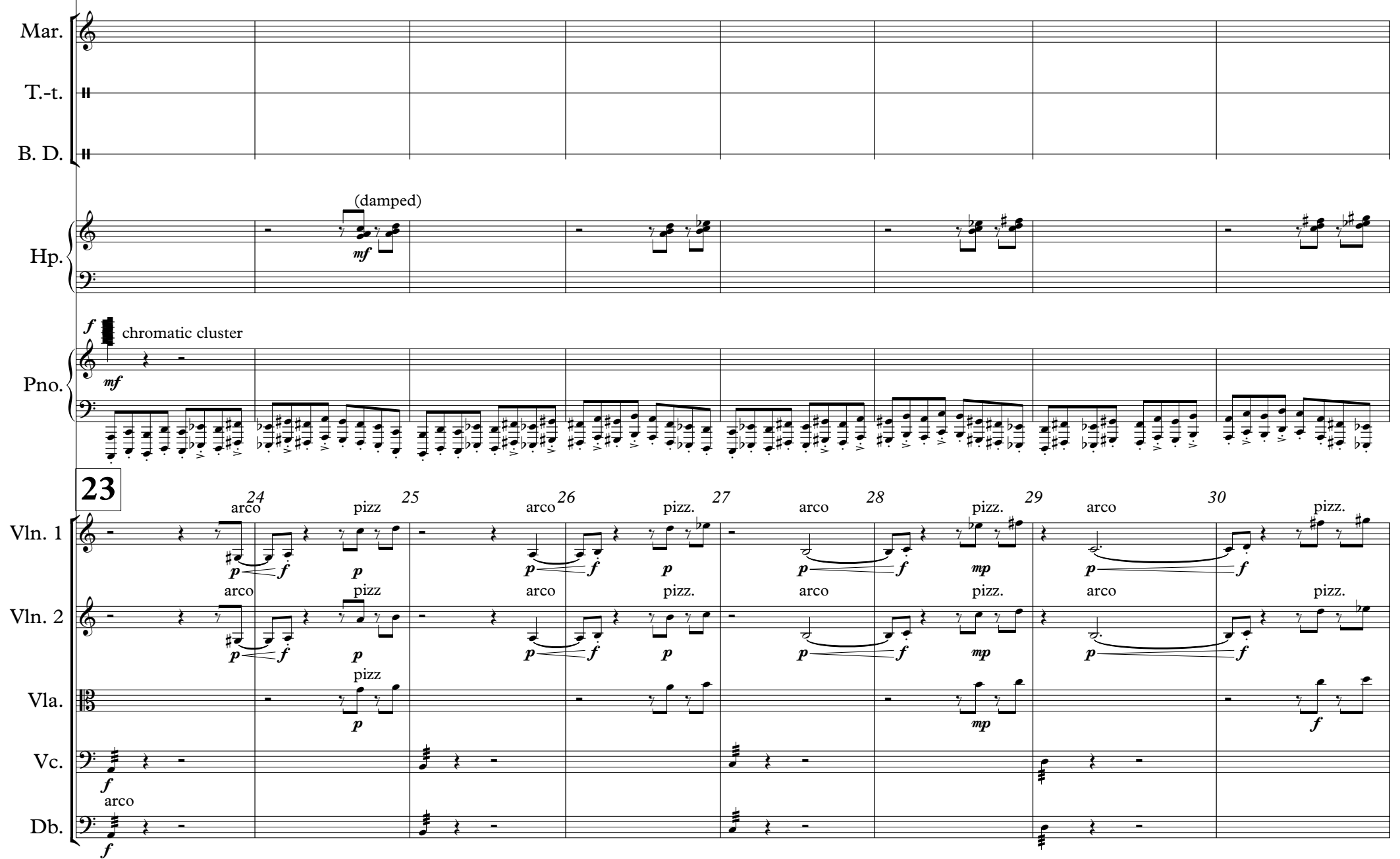

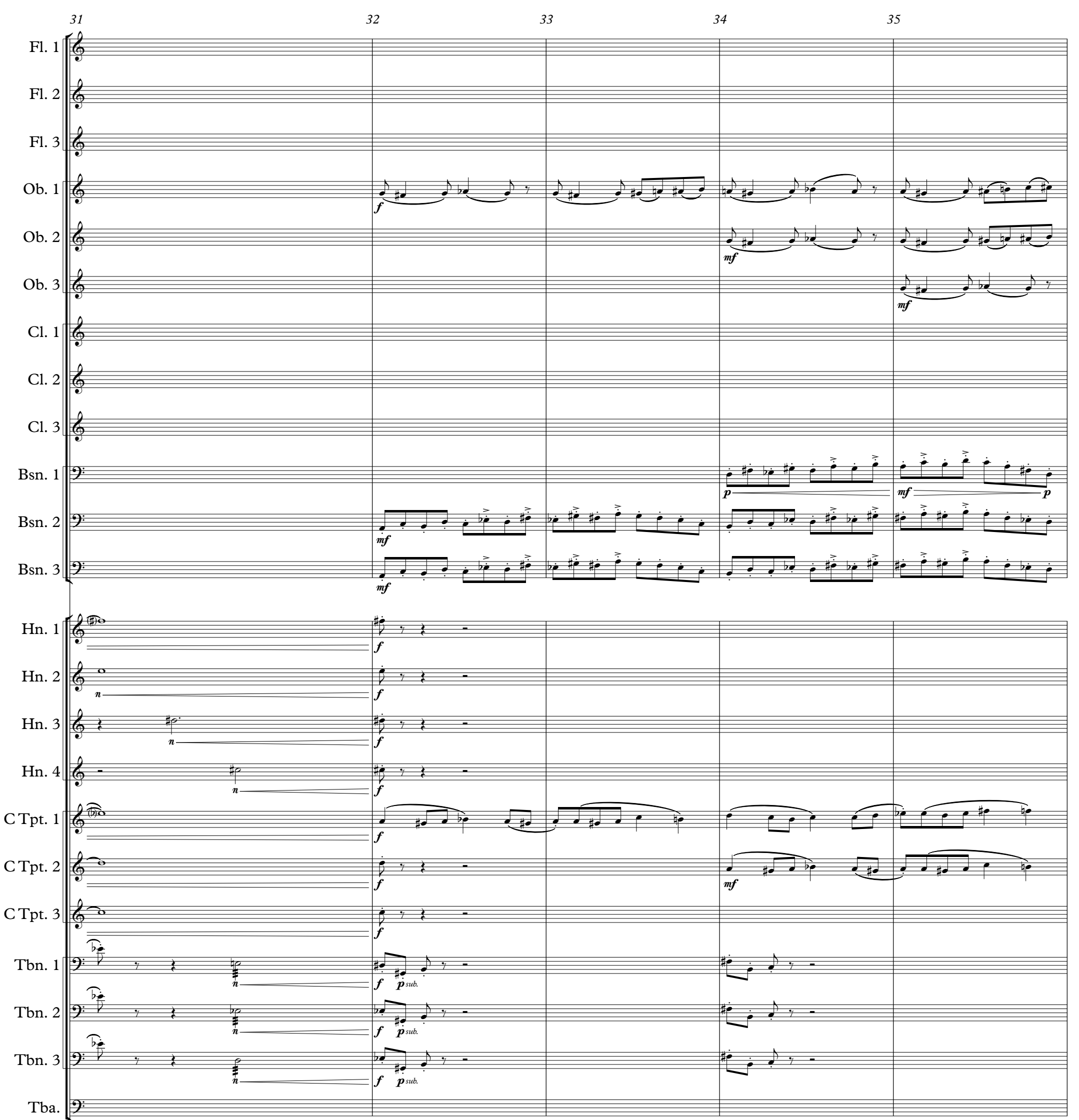

Timp.
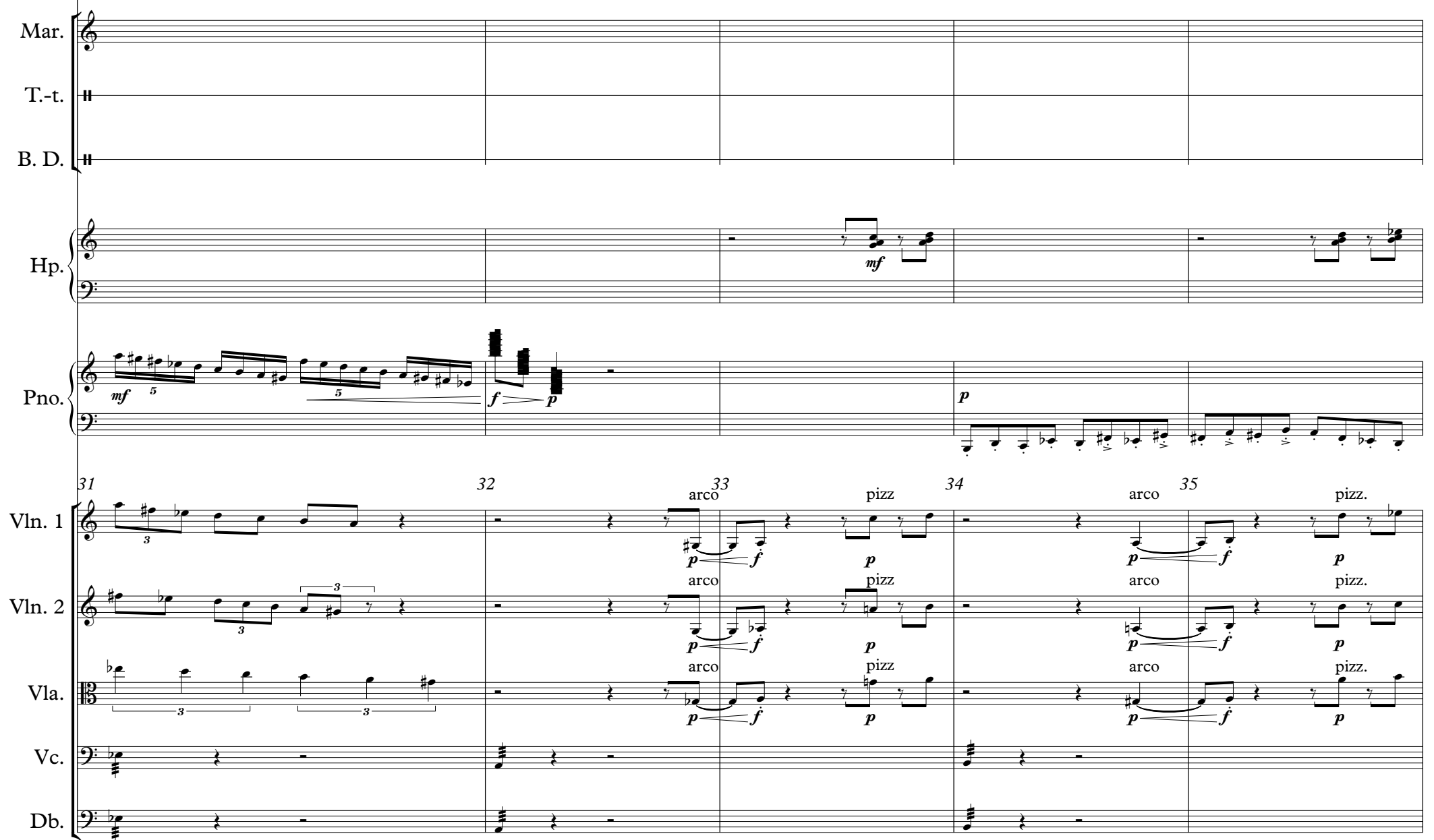

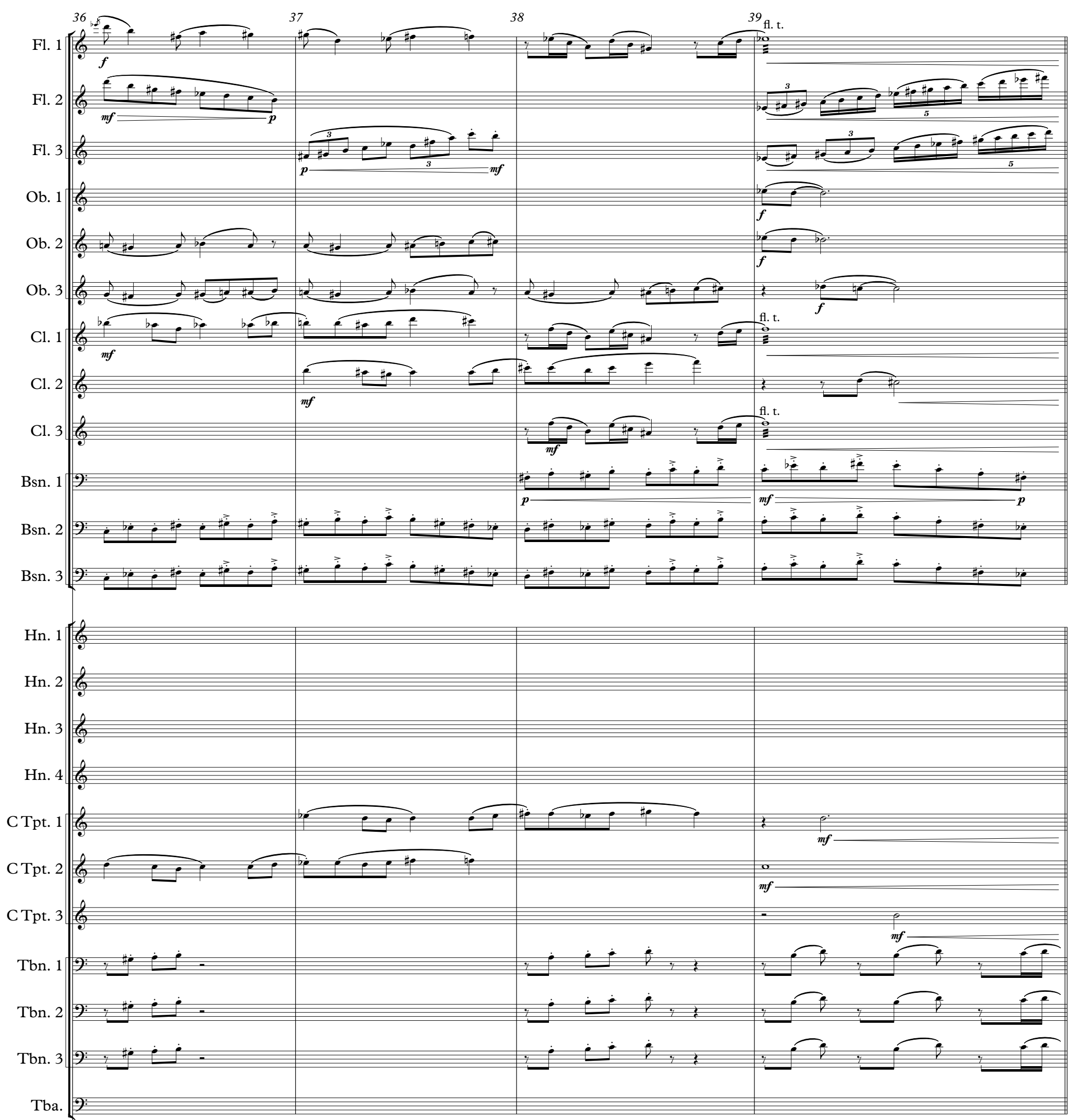

Timp.

Mar. B. D.

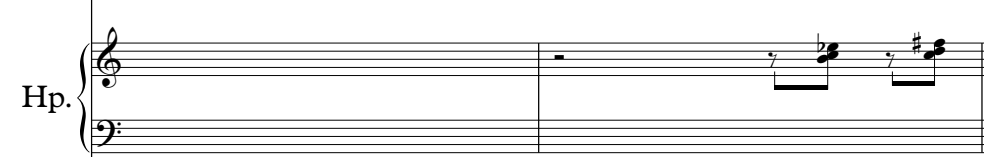

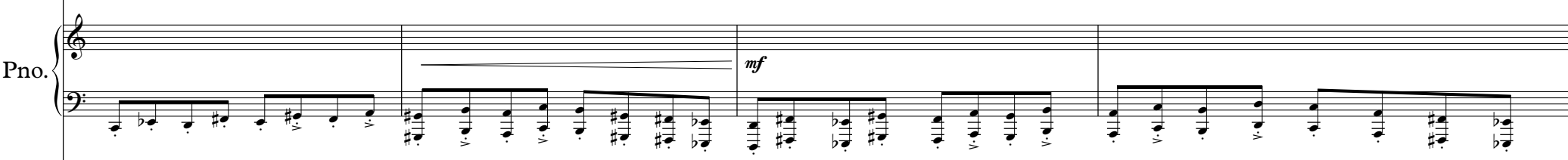

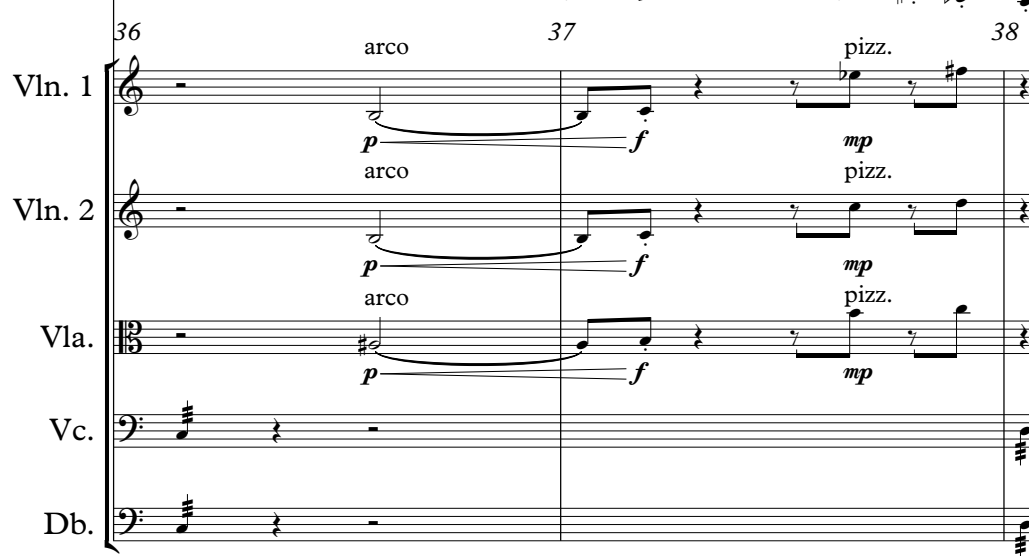


40 Poco piu mosso ca. $d=120$, anxious
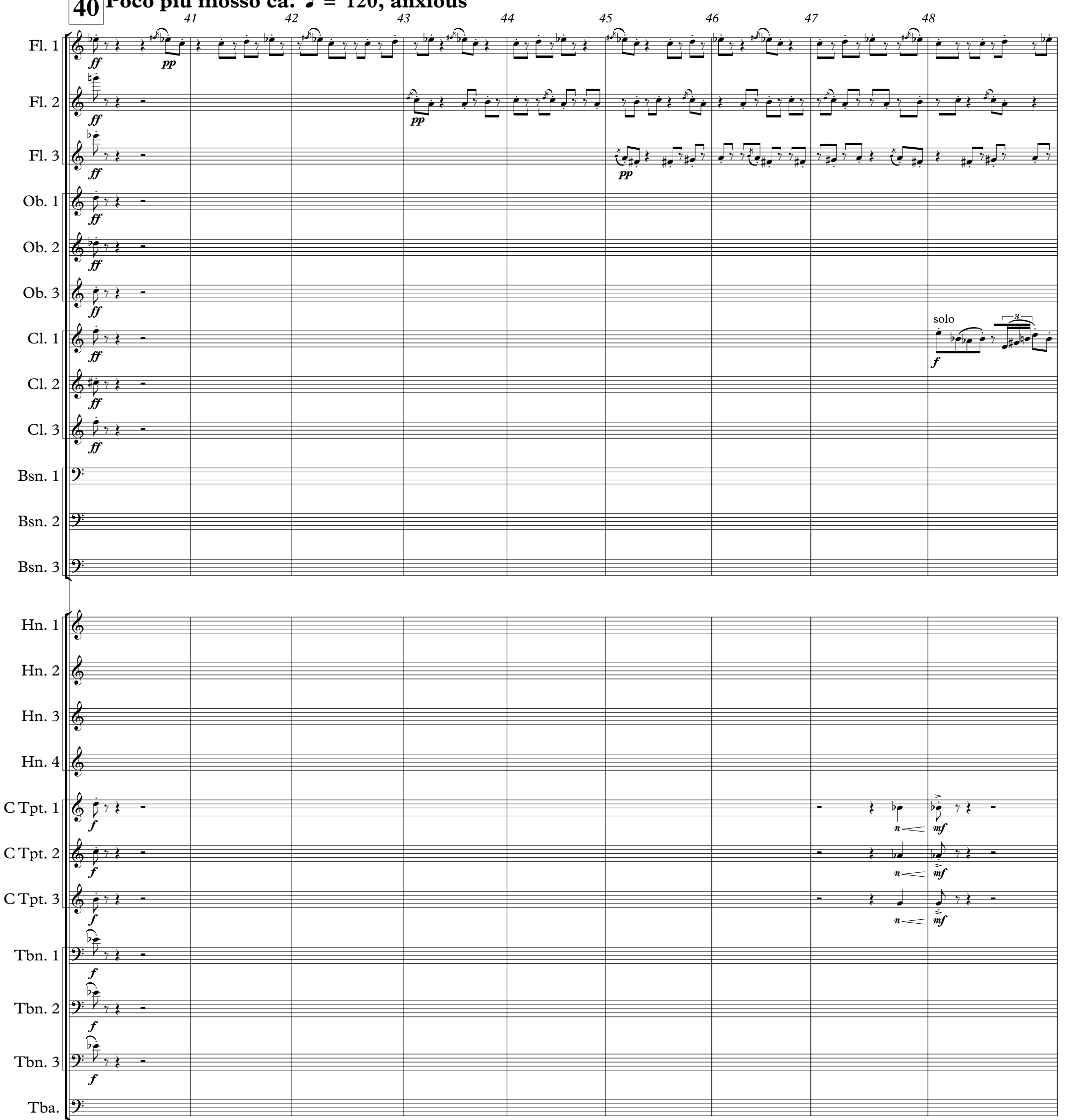

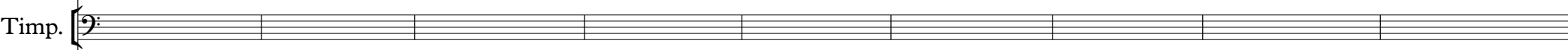
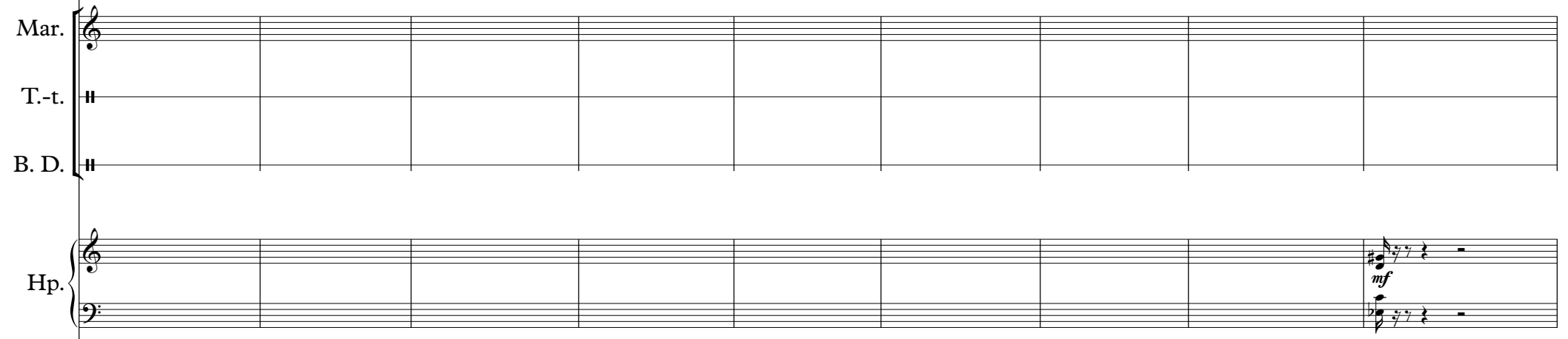

Pno.

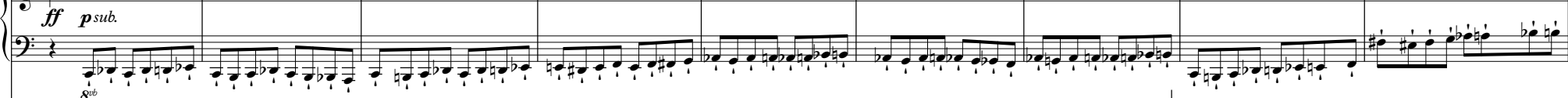

40 Poco piu mosso ca. $\underset{42}{d}=120, \underset{43}{\text { anxious }}$

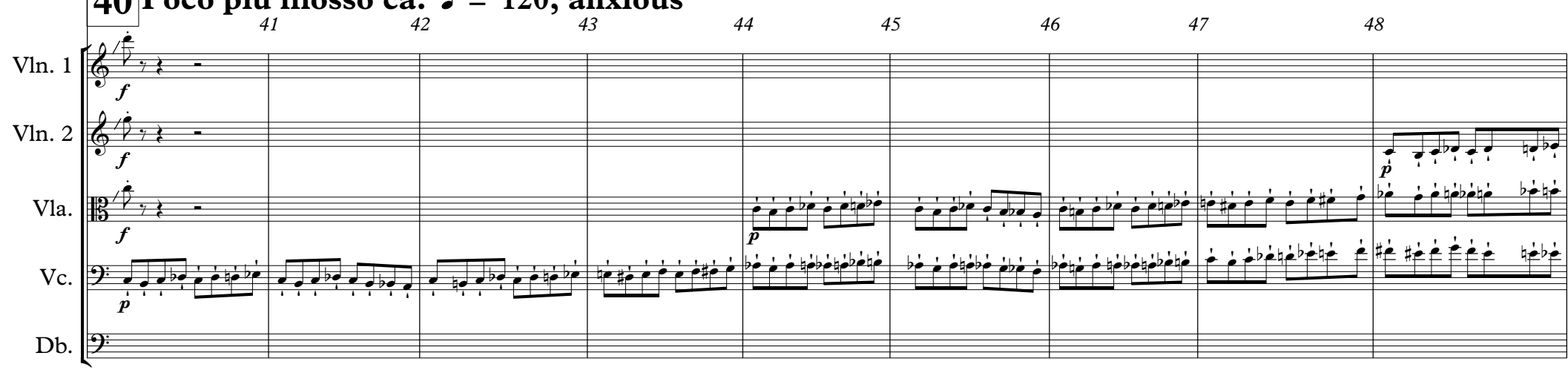



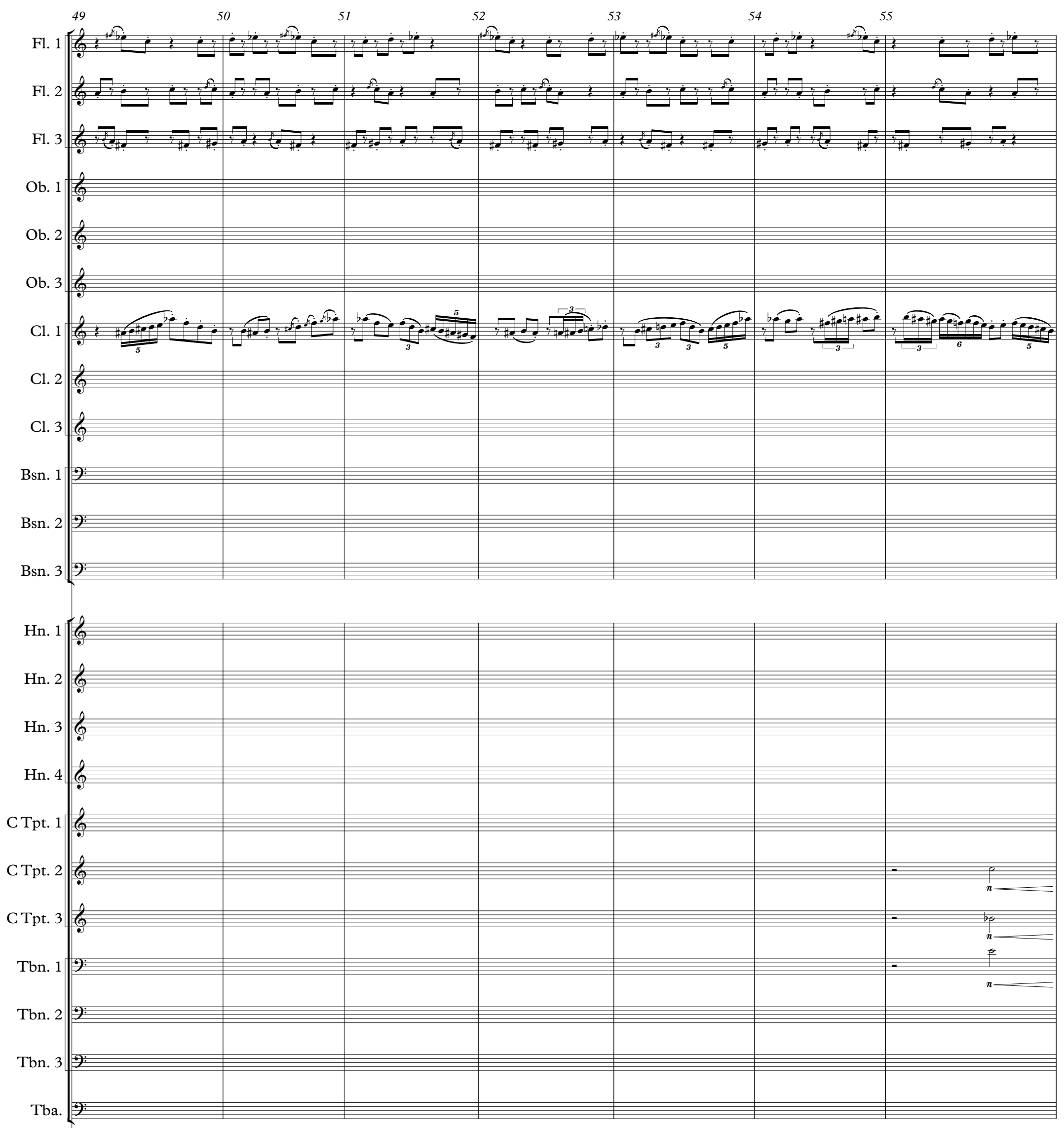

Timp. 0 核
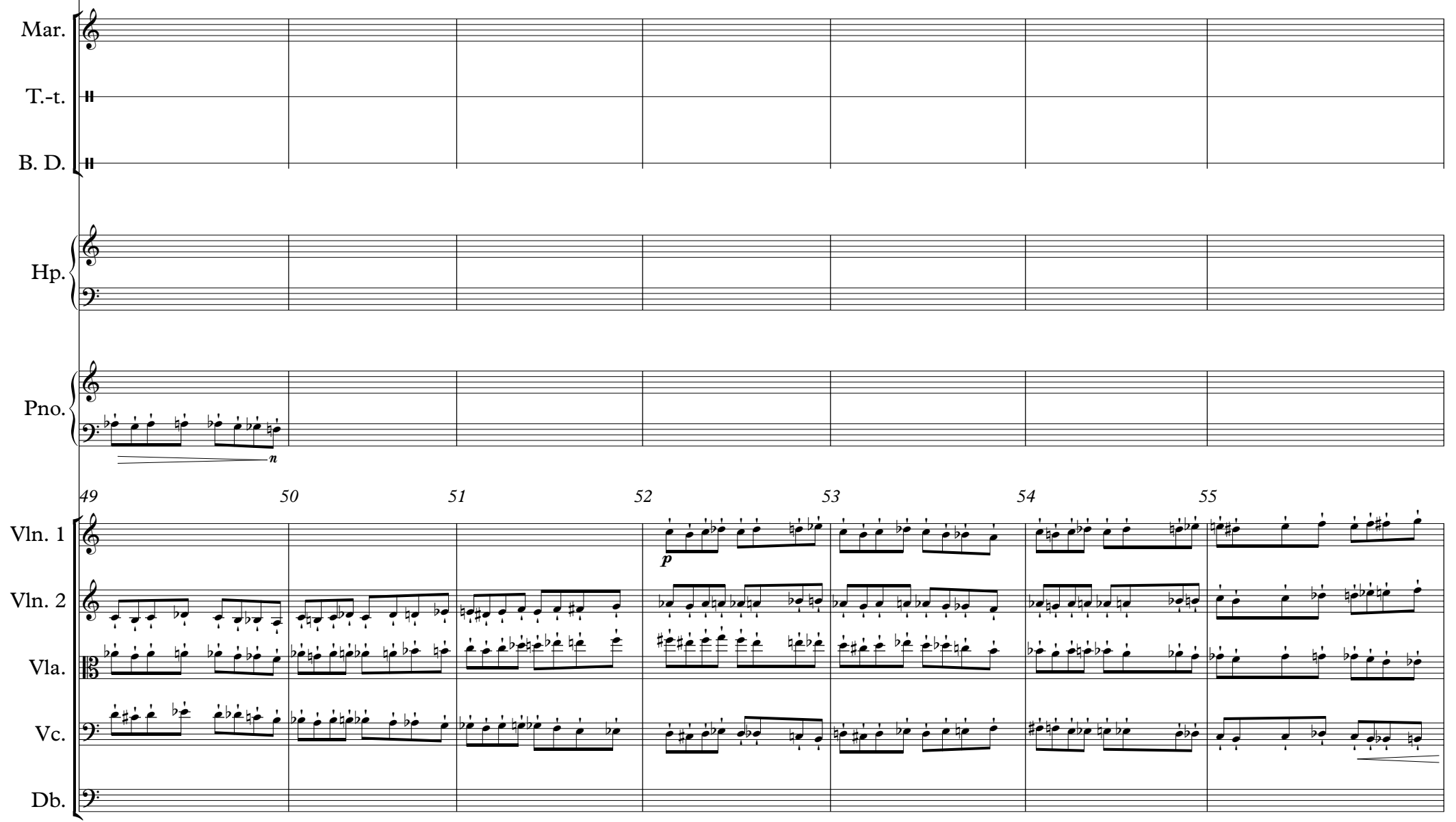

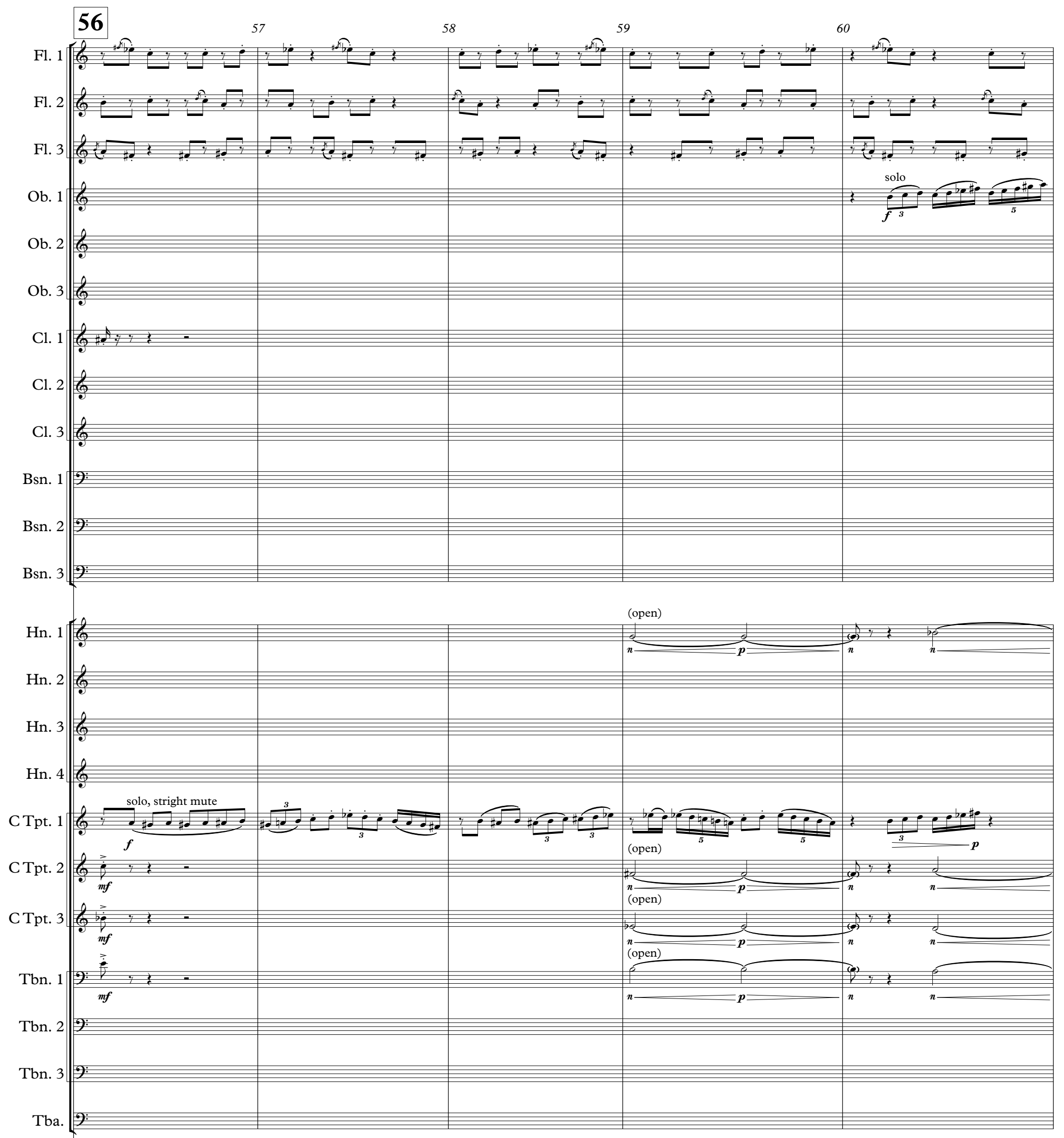

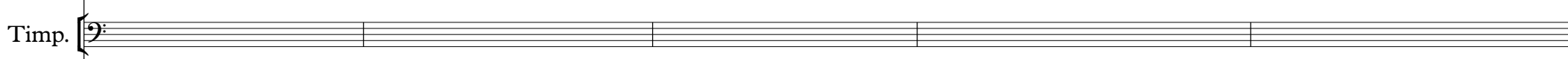
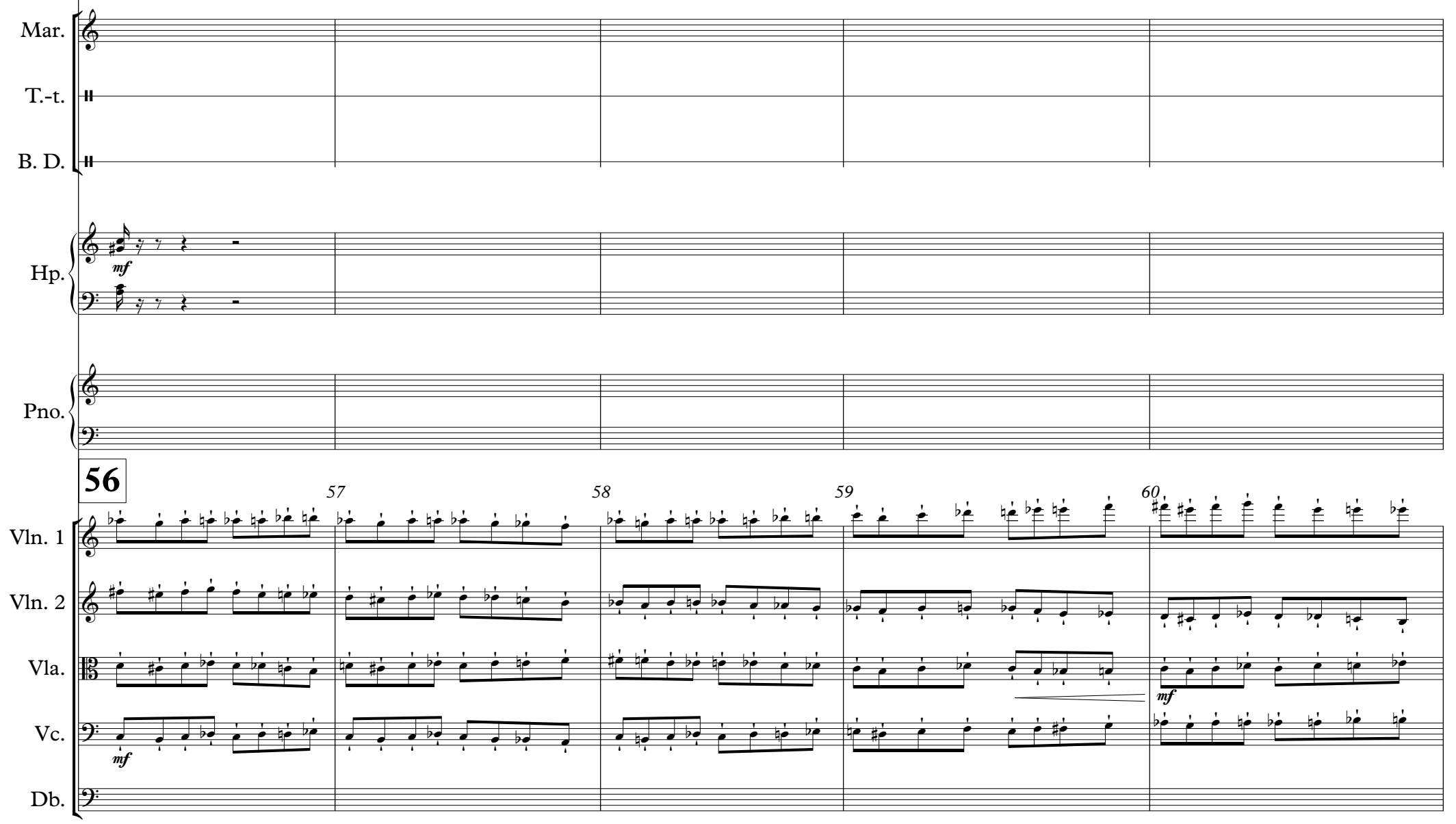

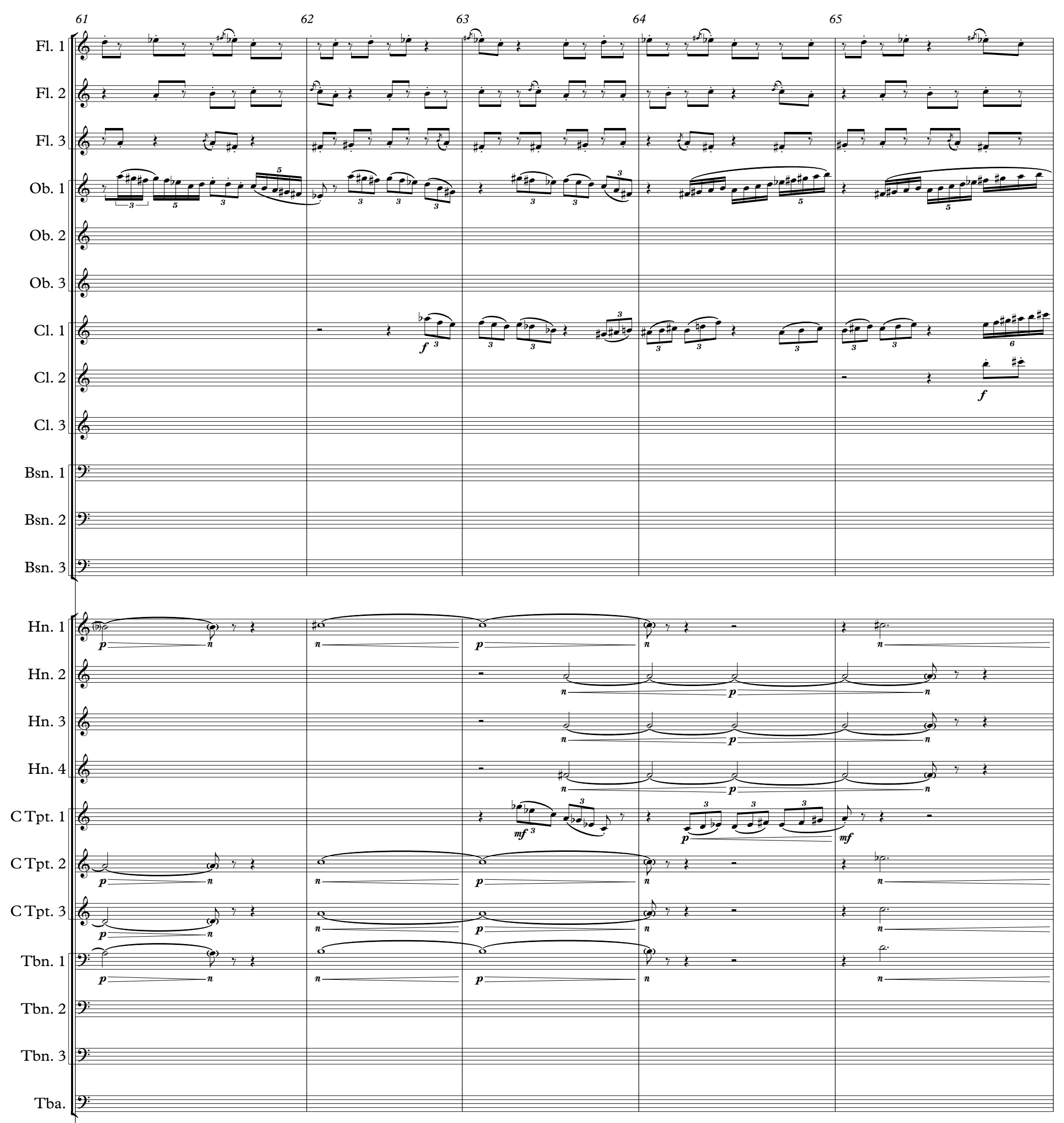

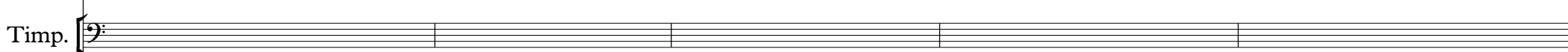
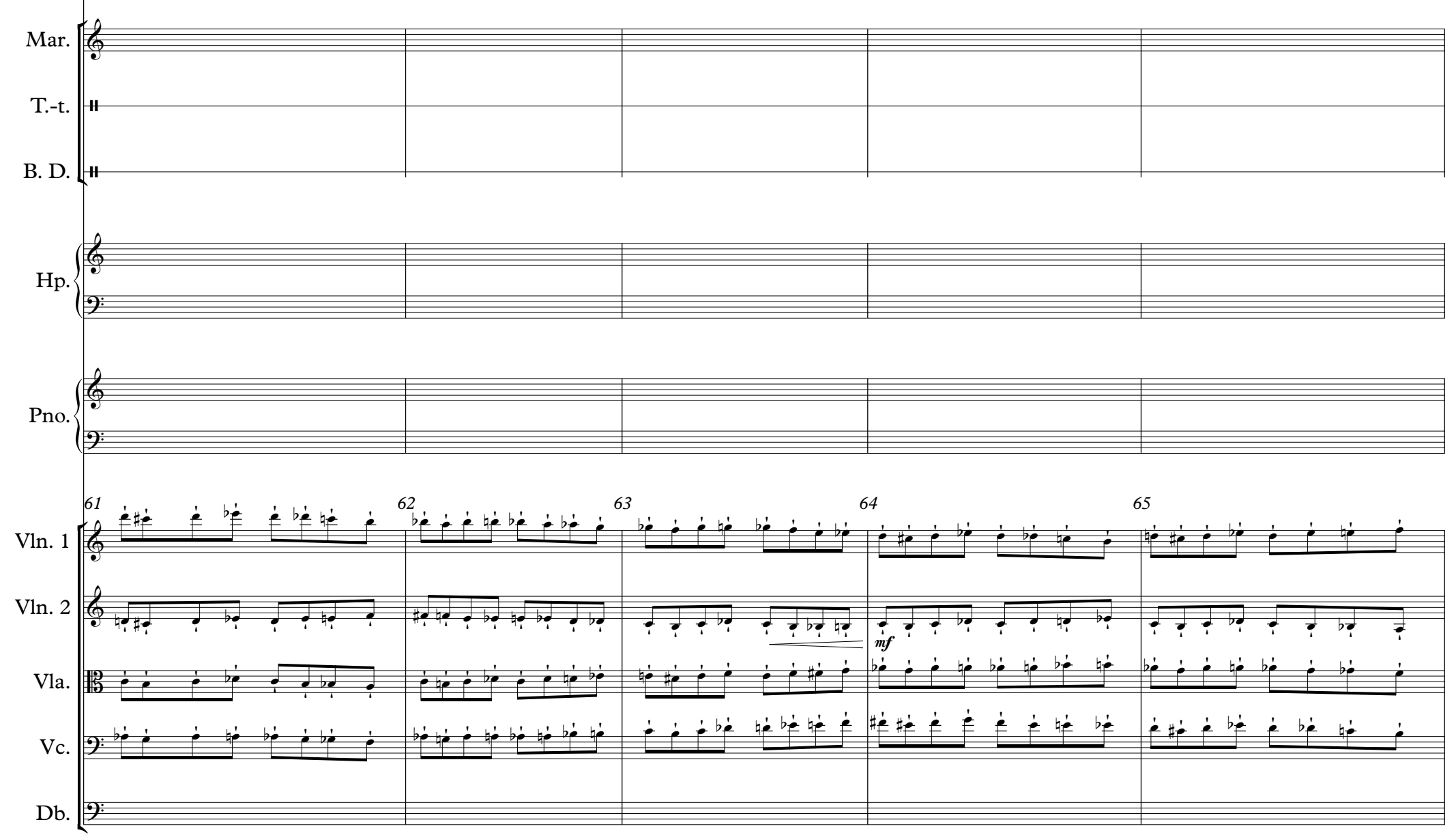

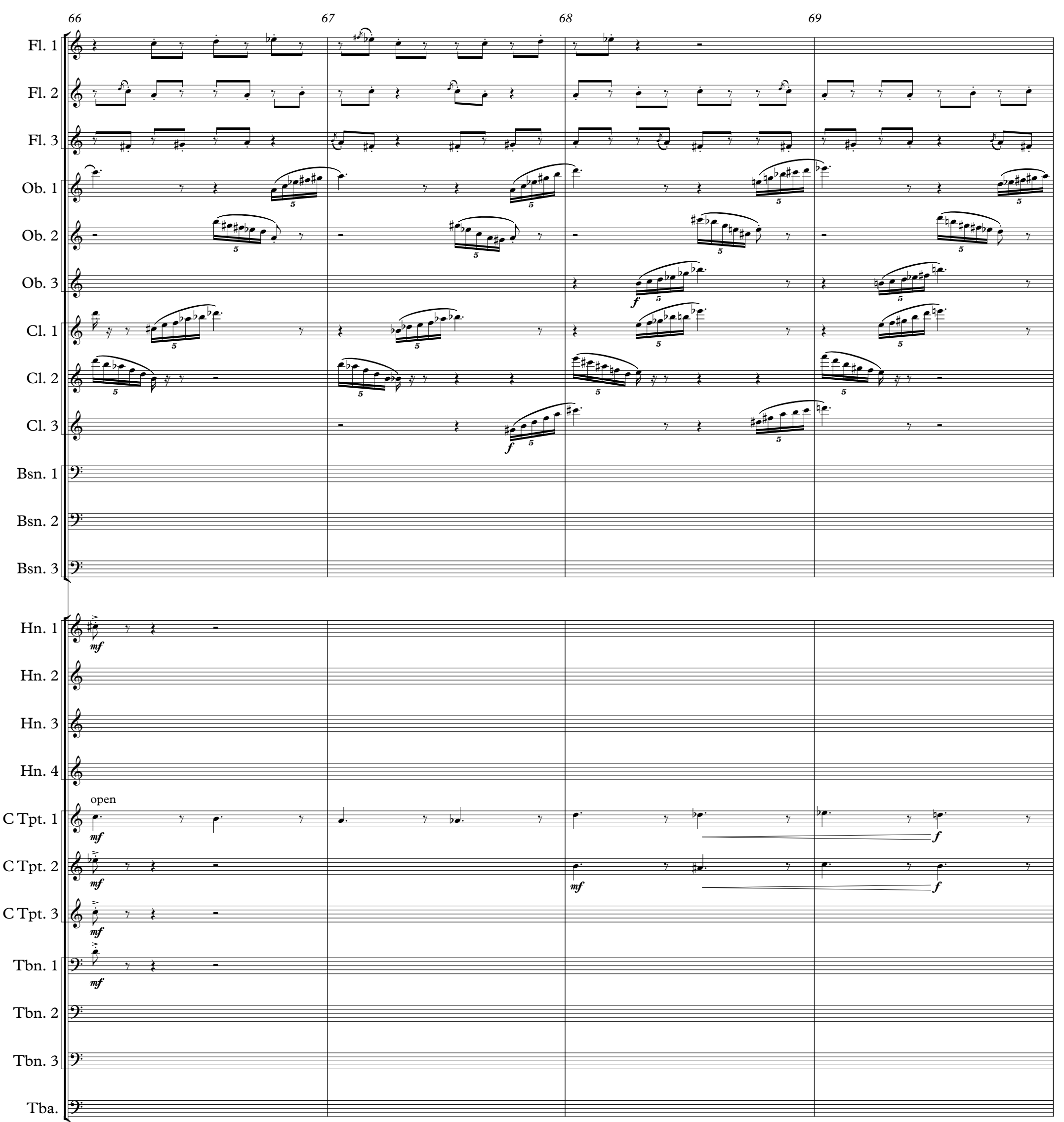

Timp.
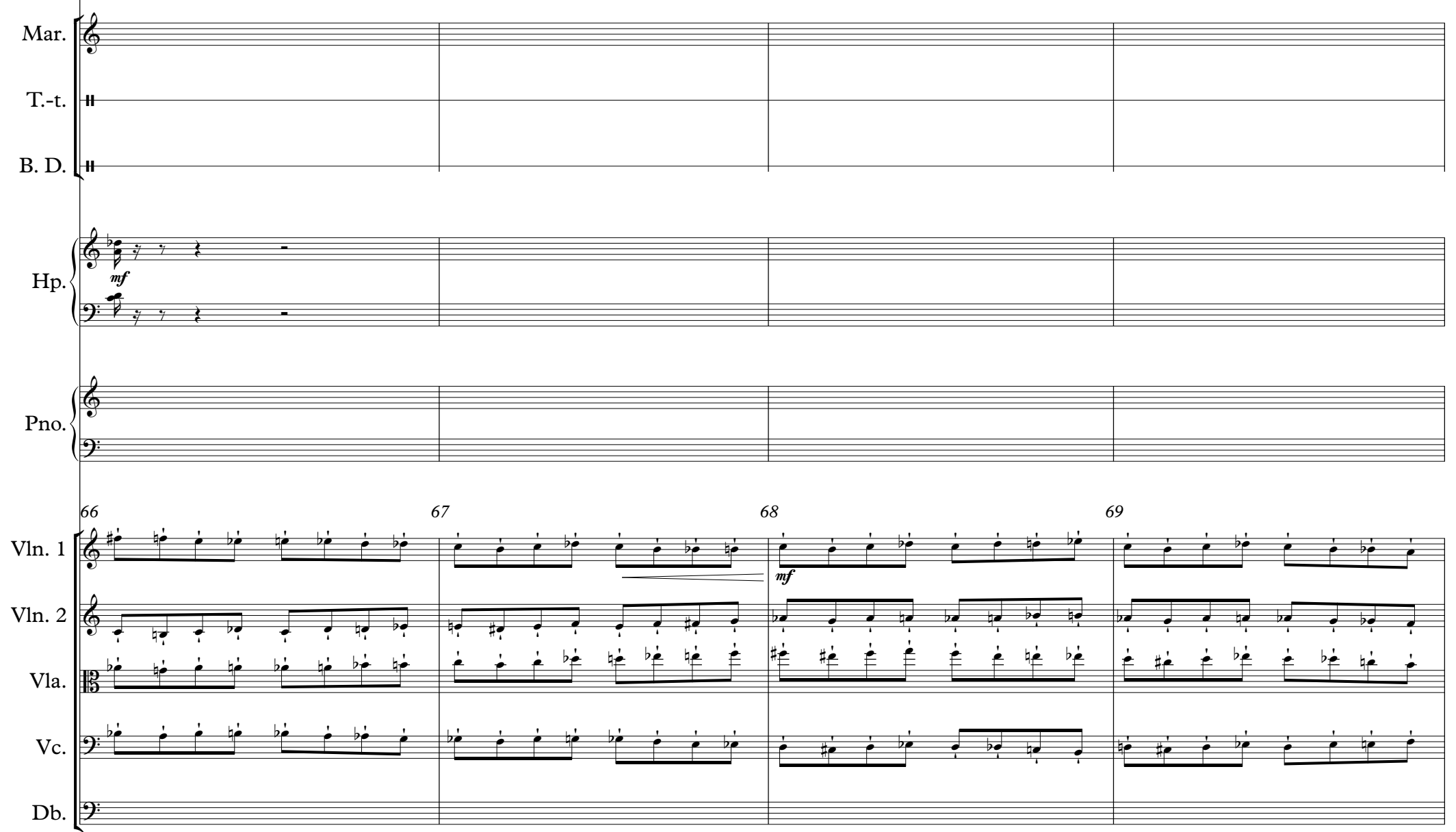

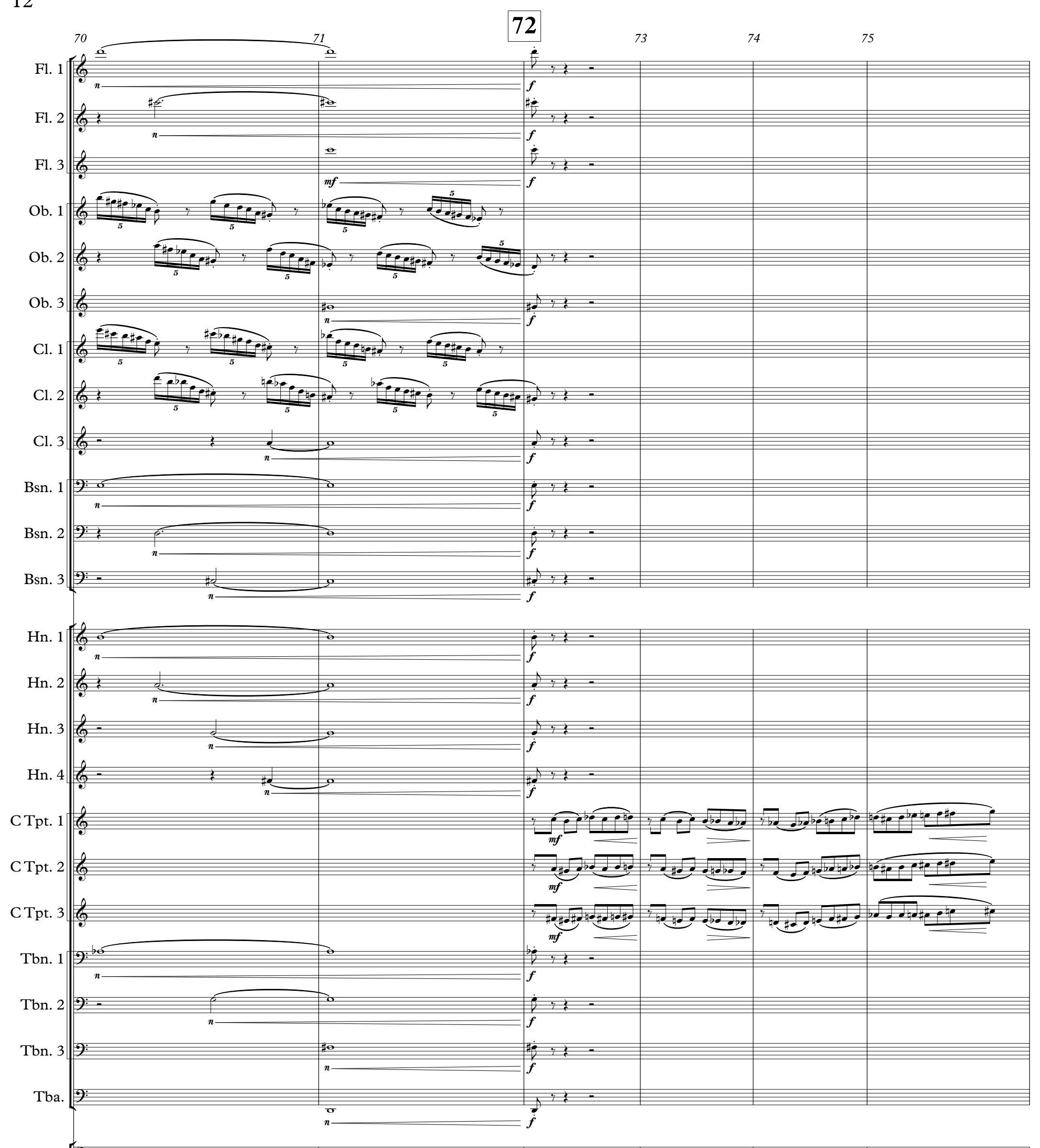

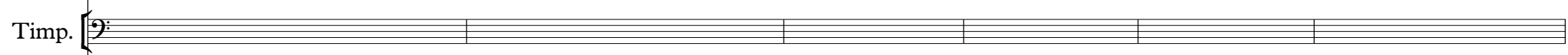
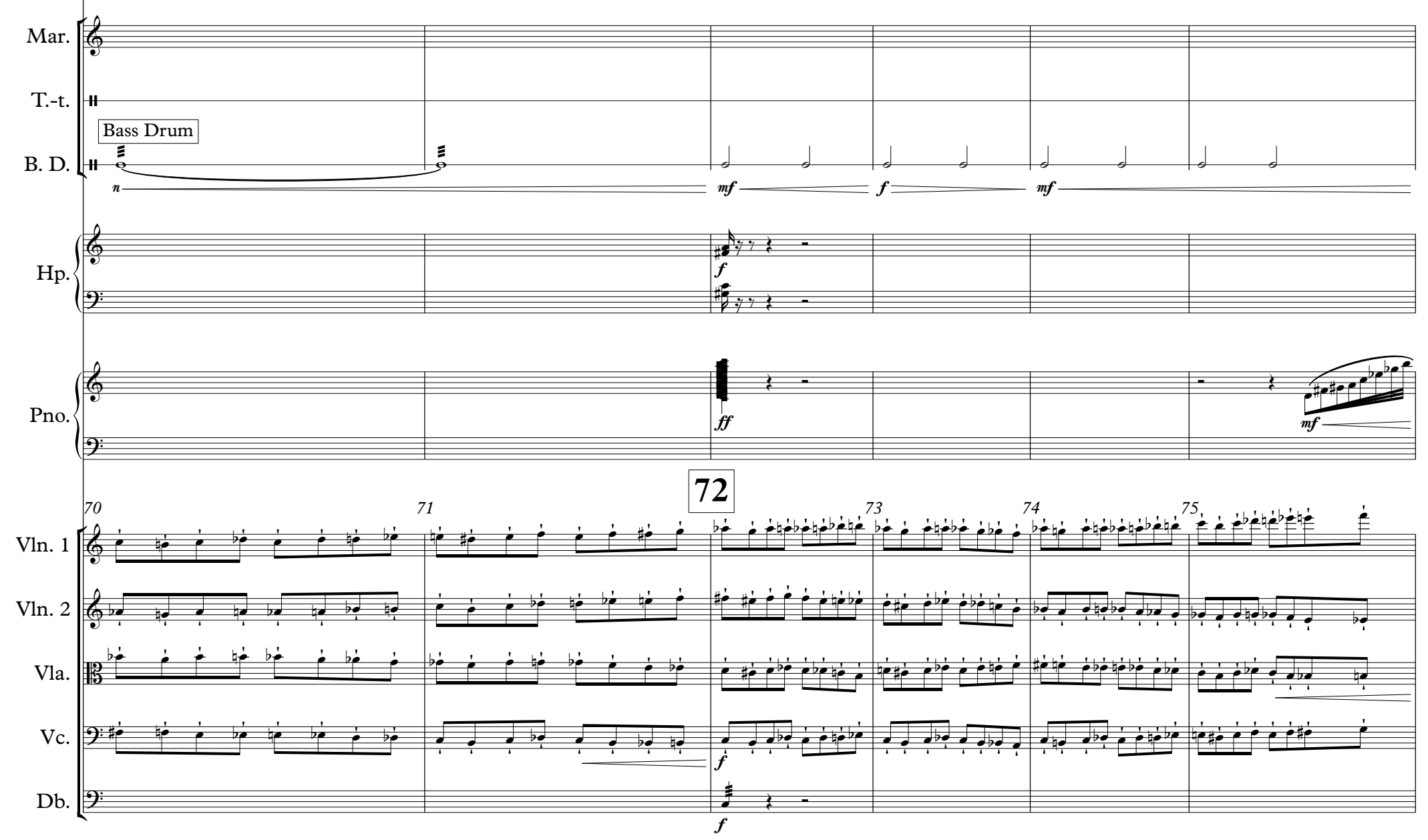

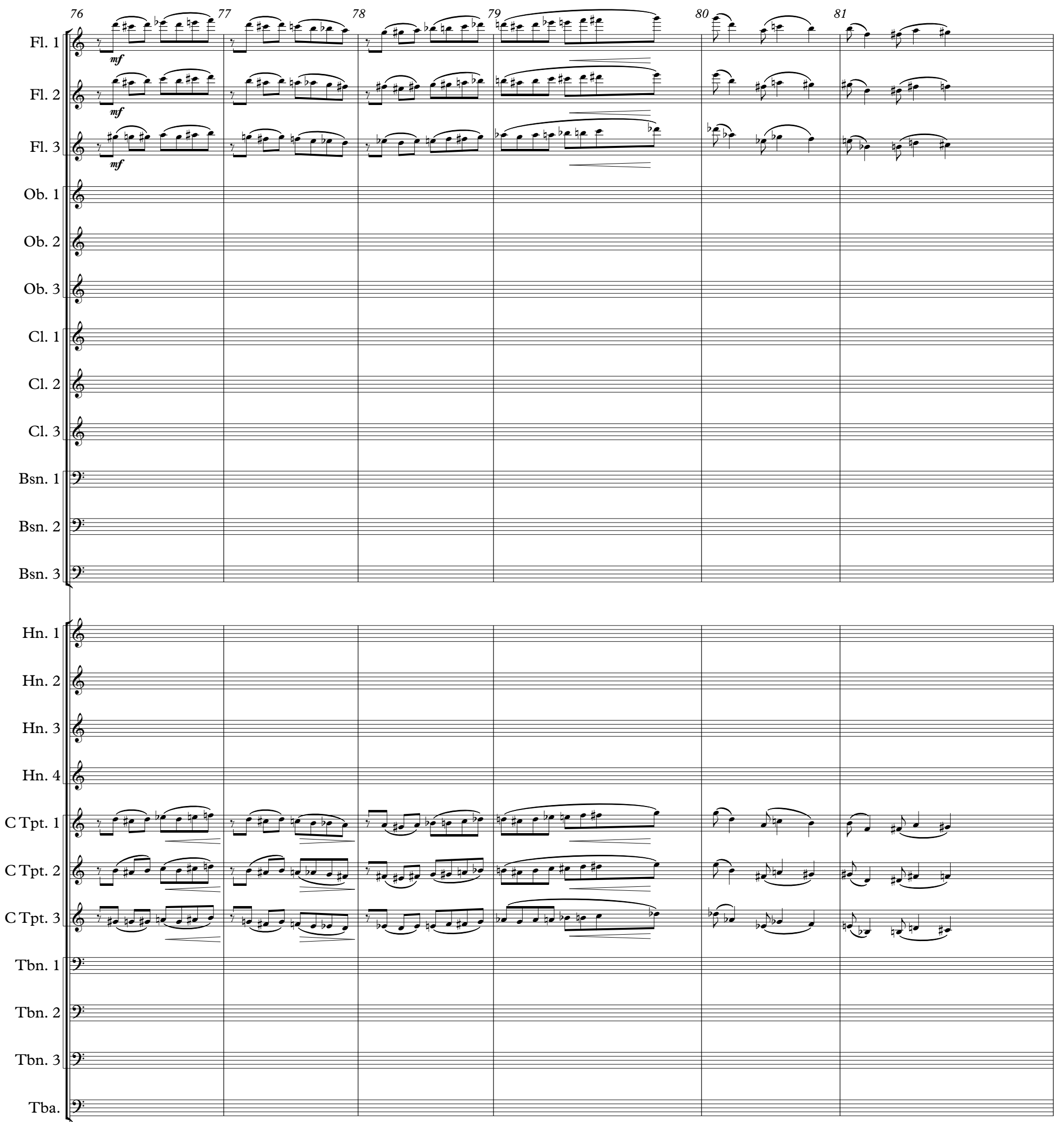

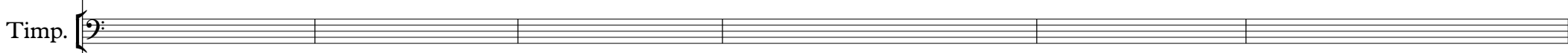
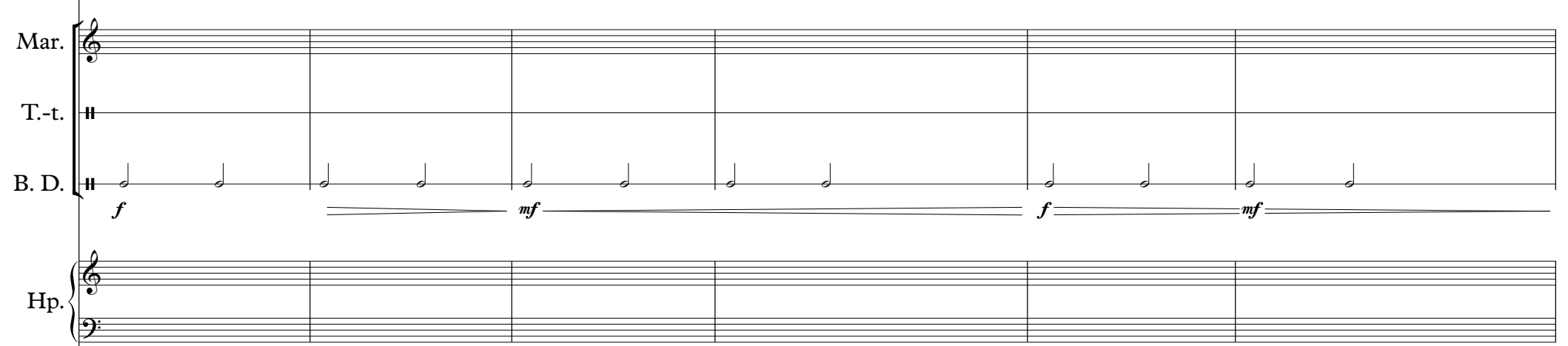

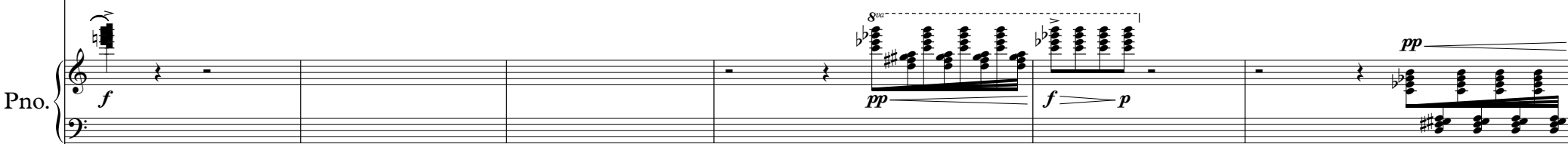

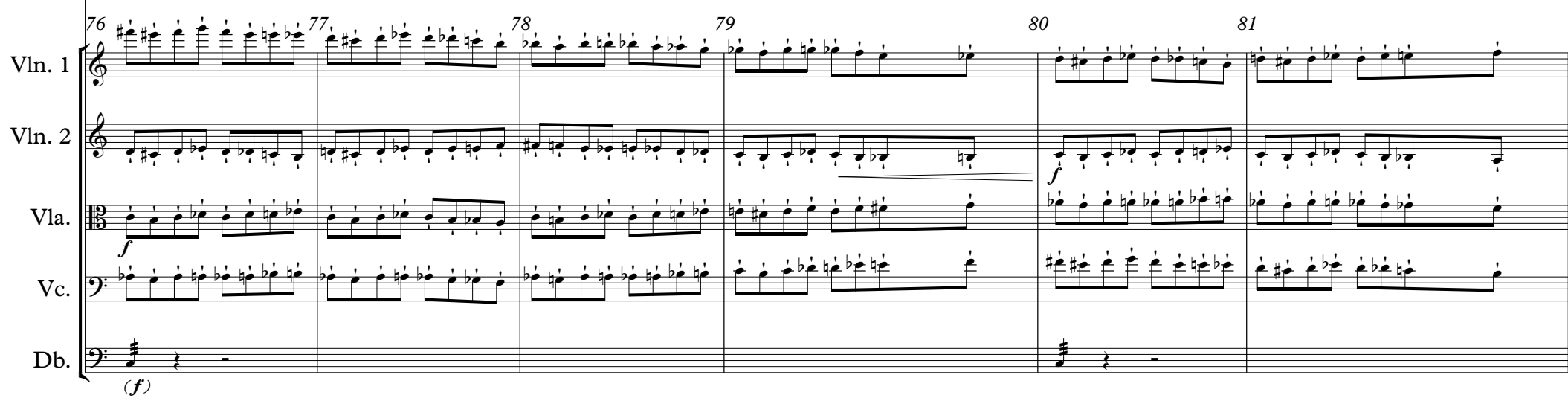



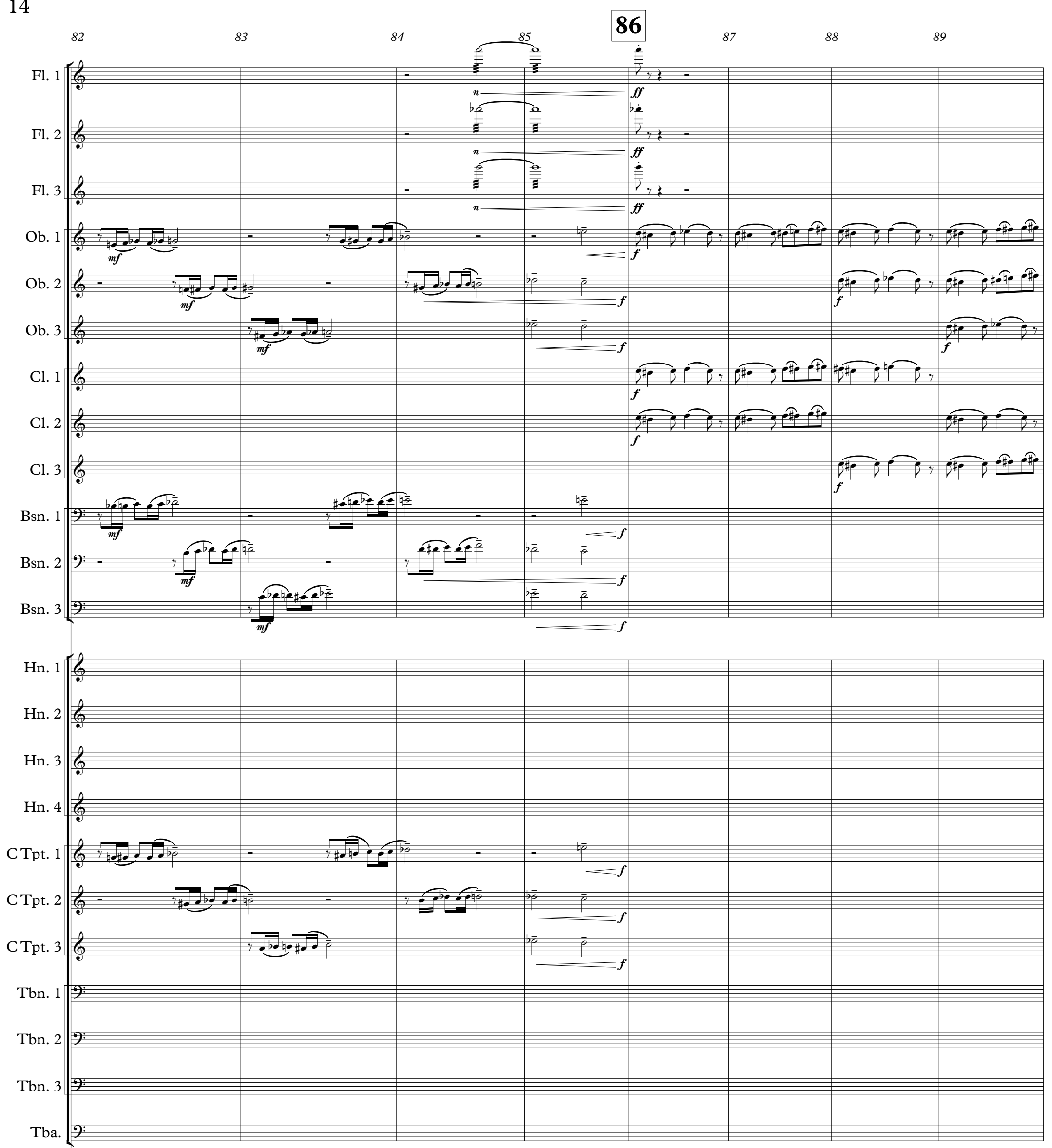

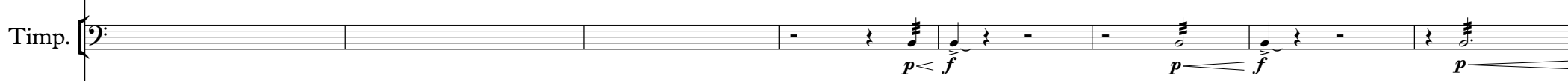
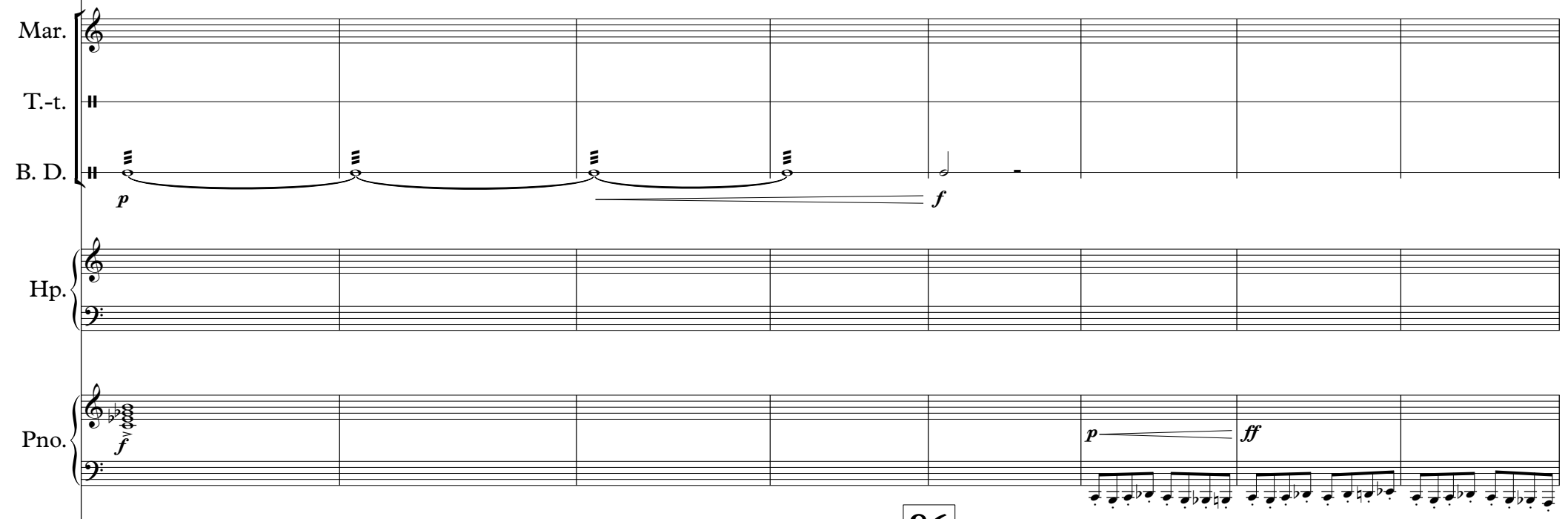

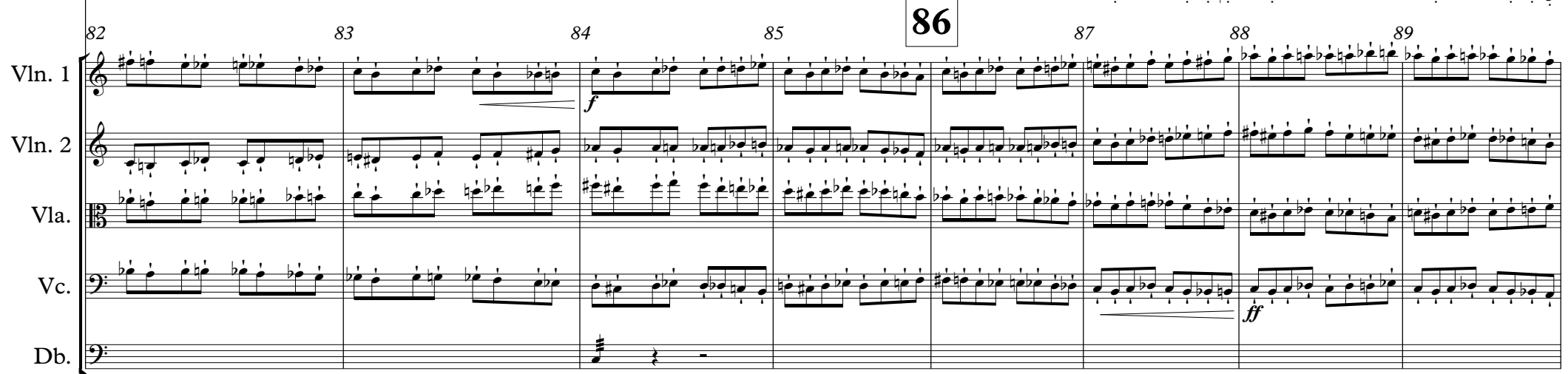



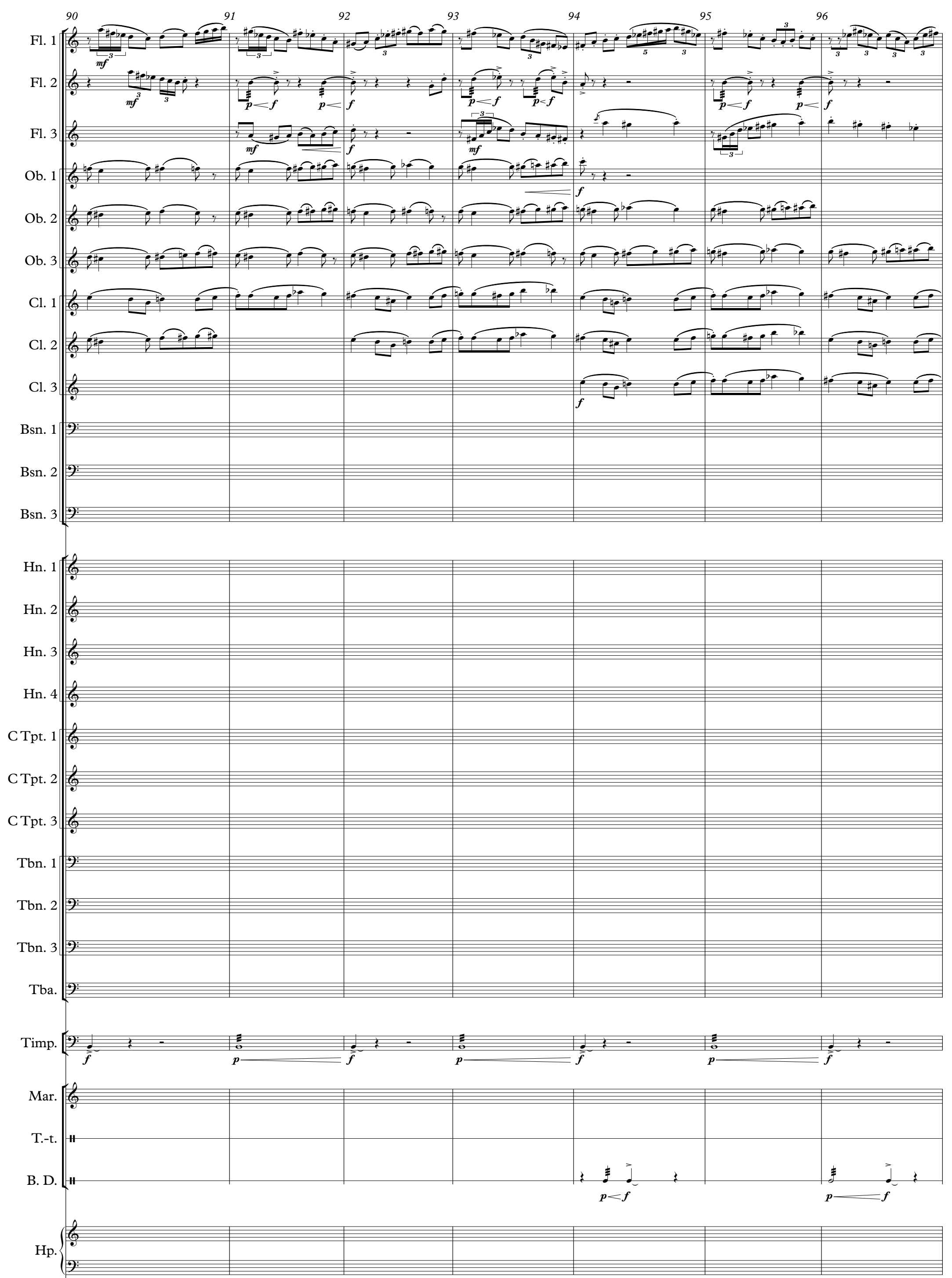

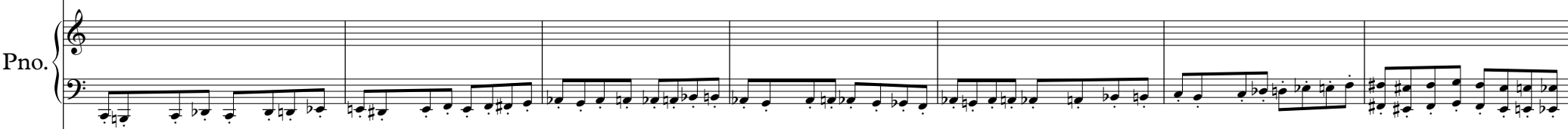

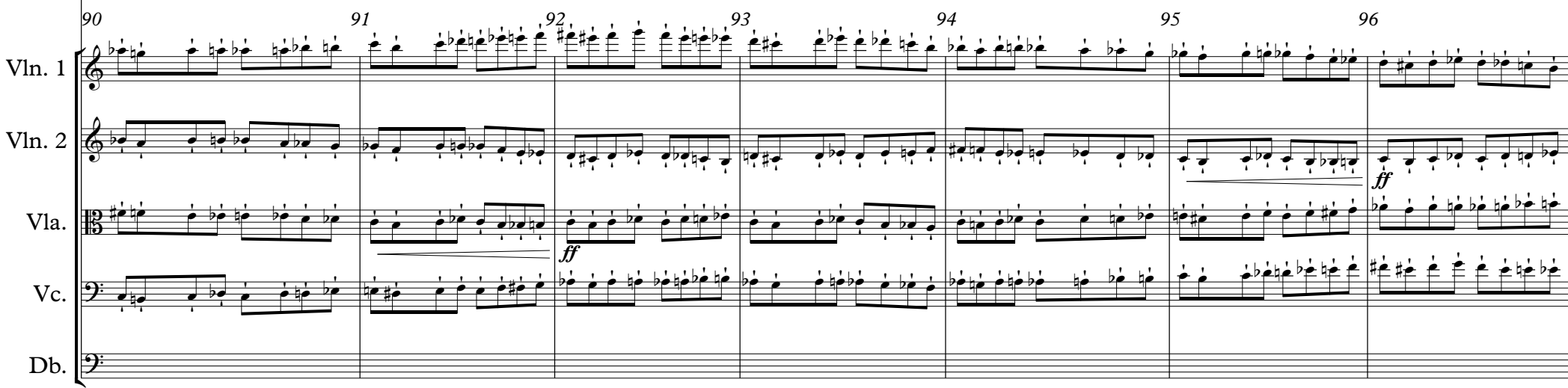



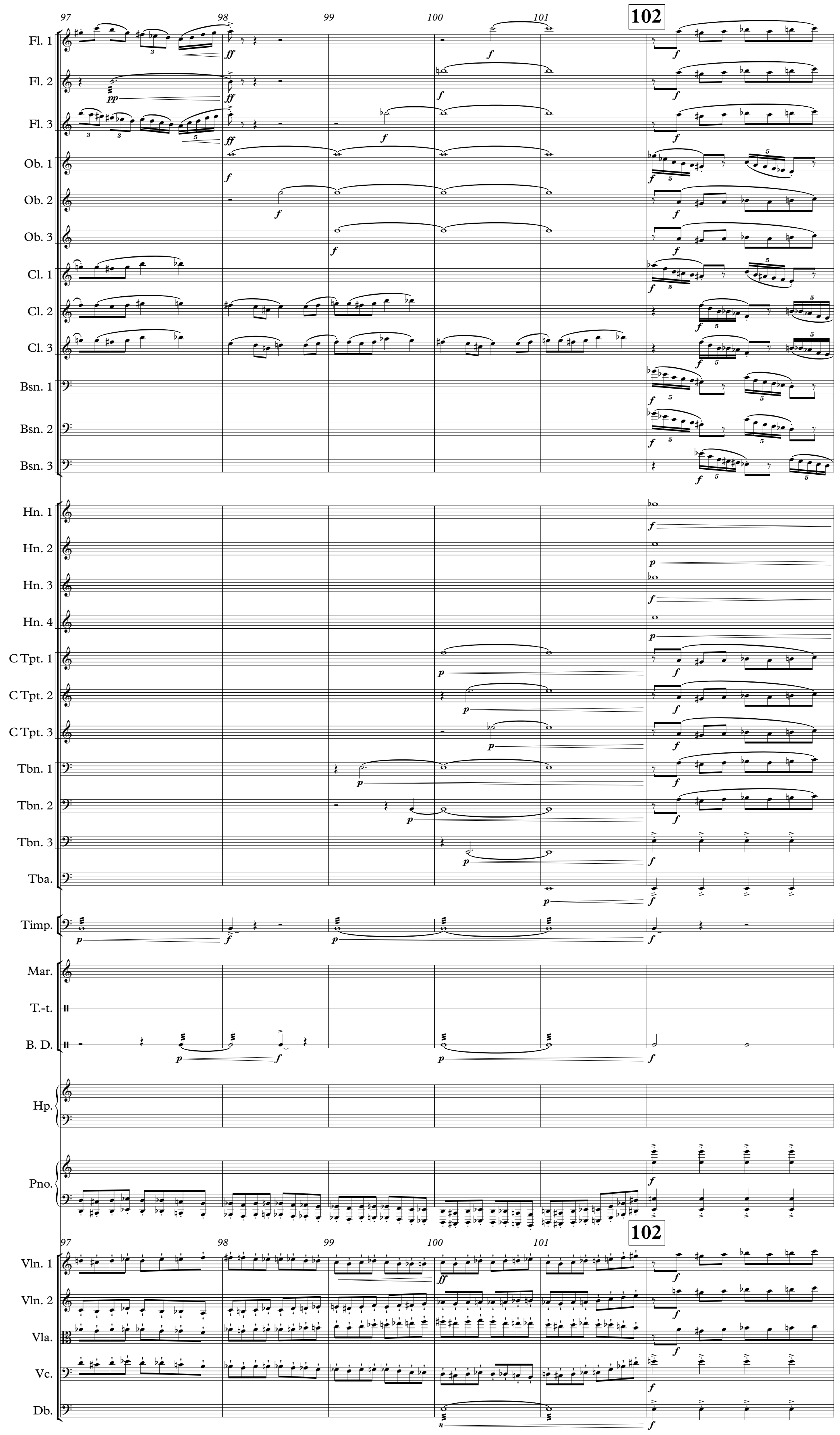

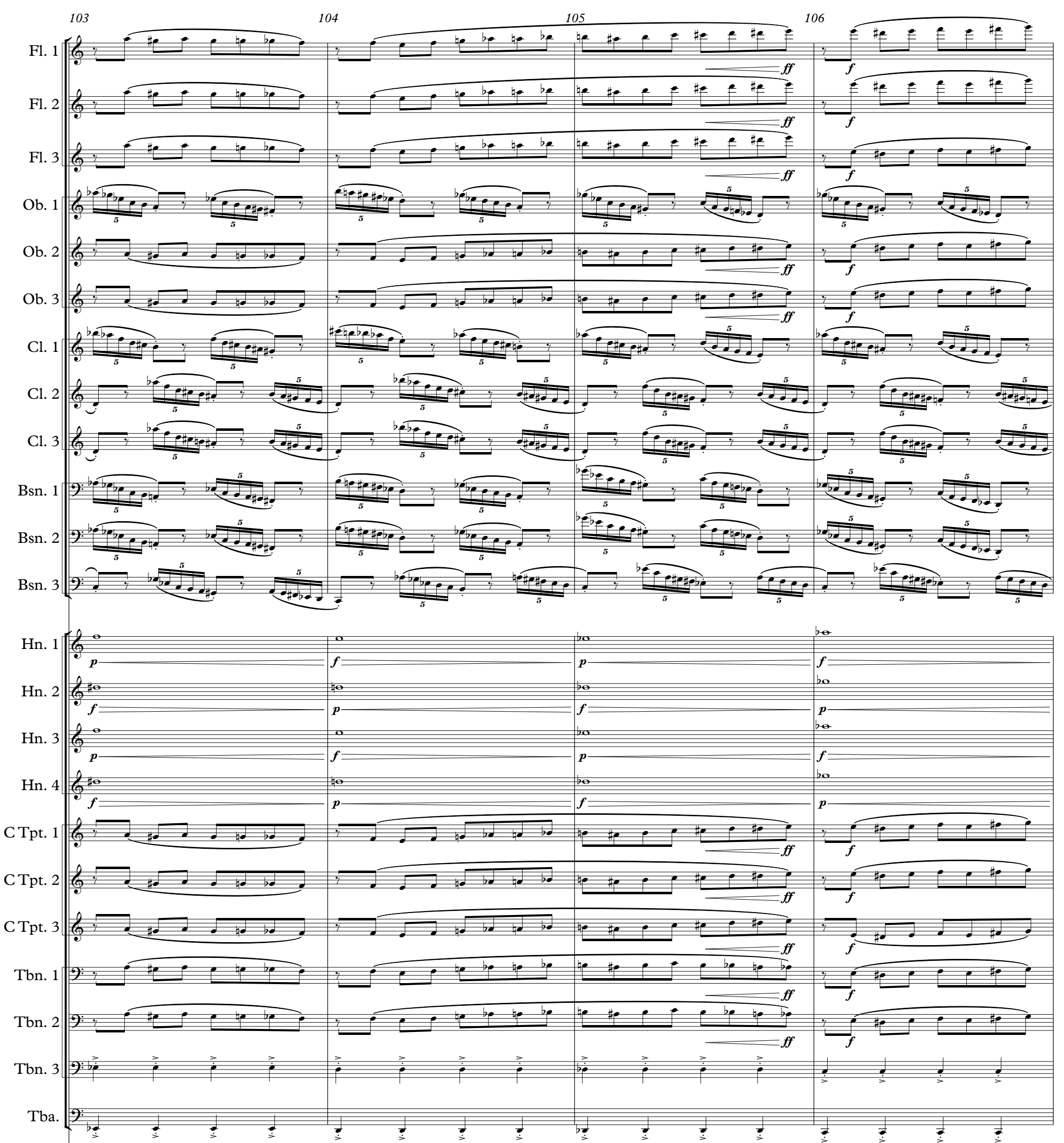

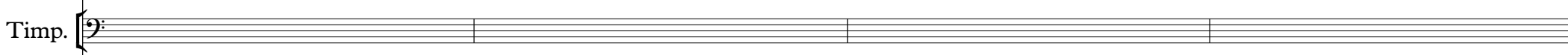

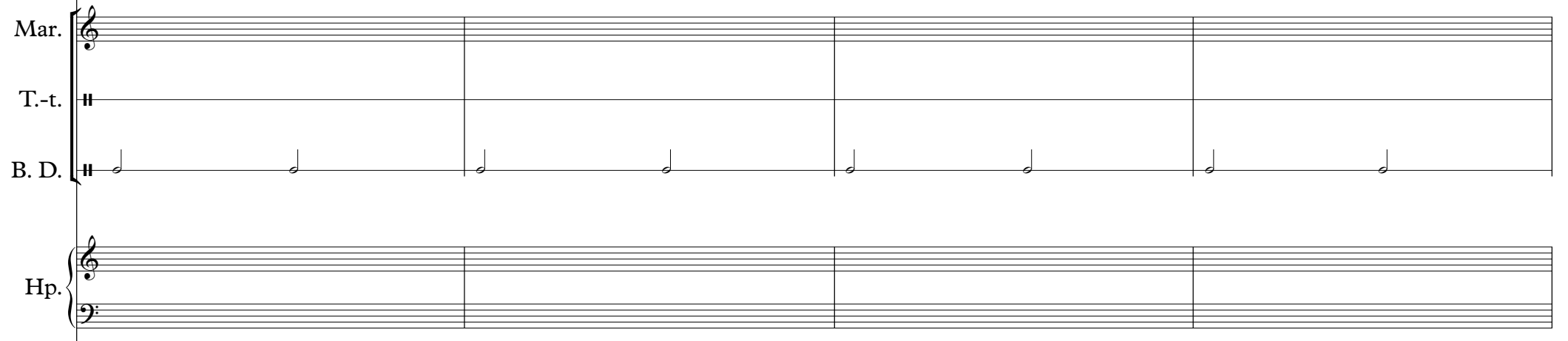

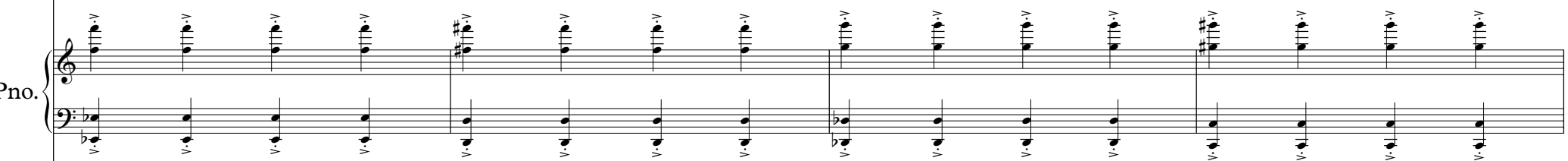

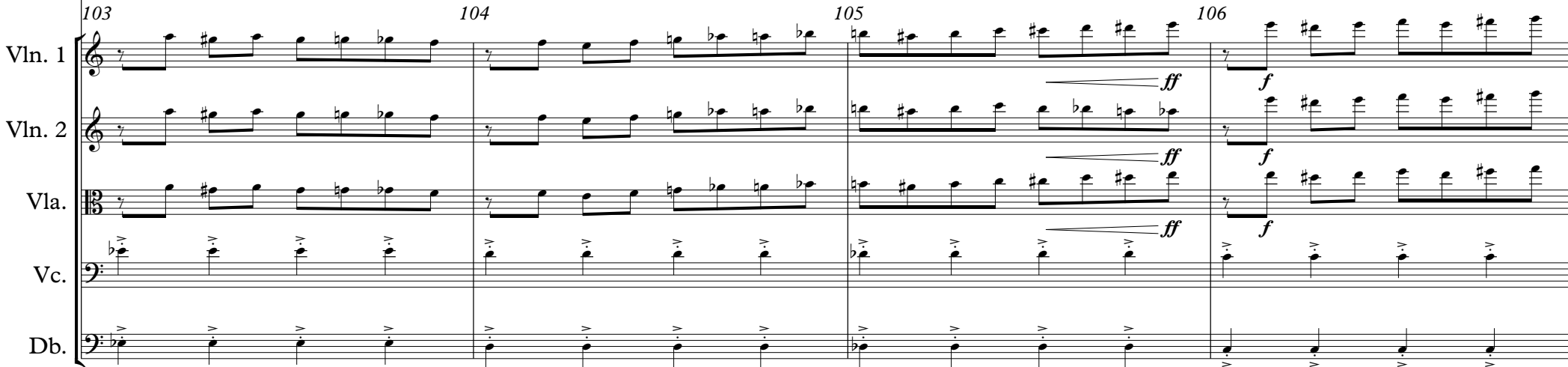



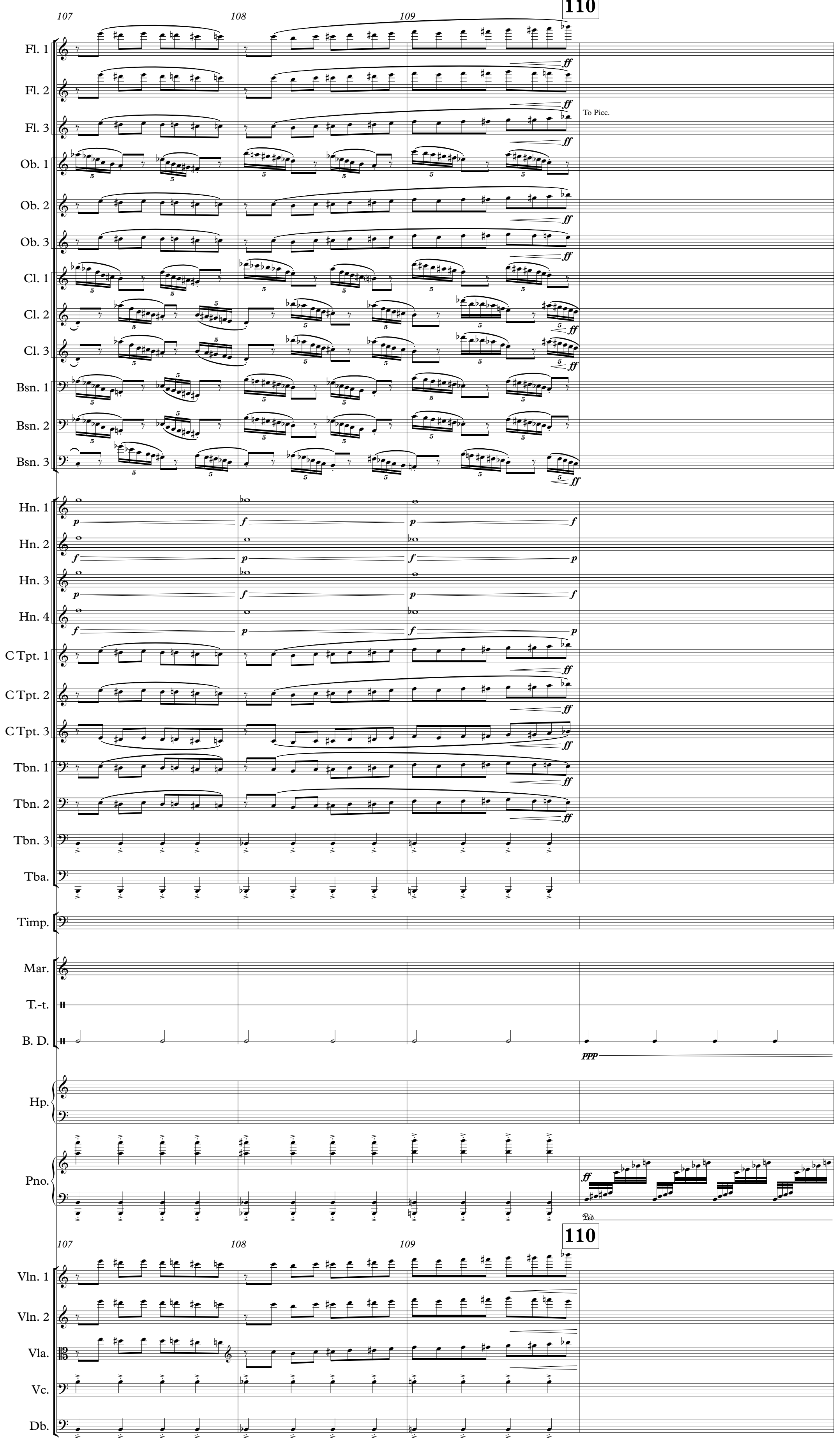

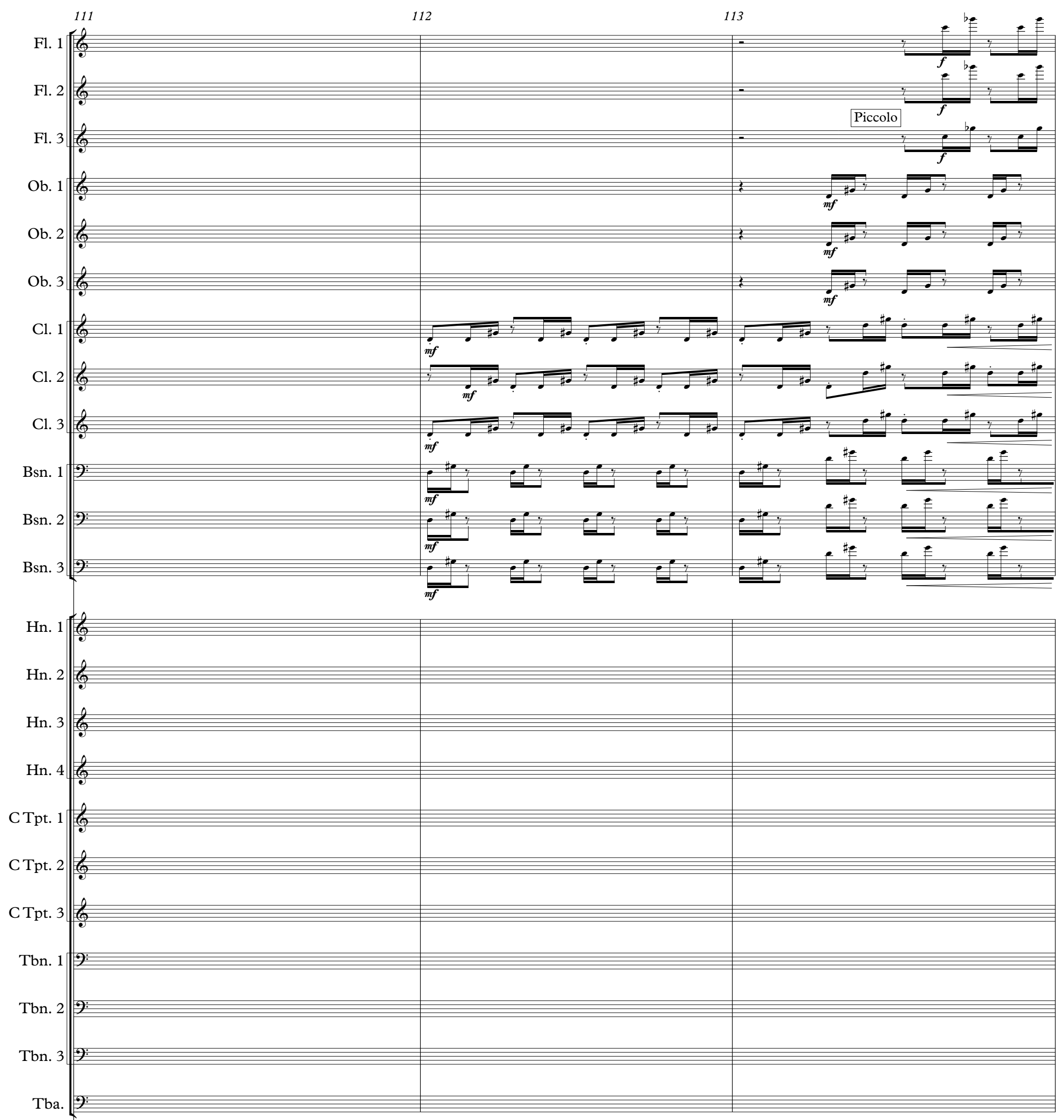

Timp.
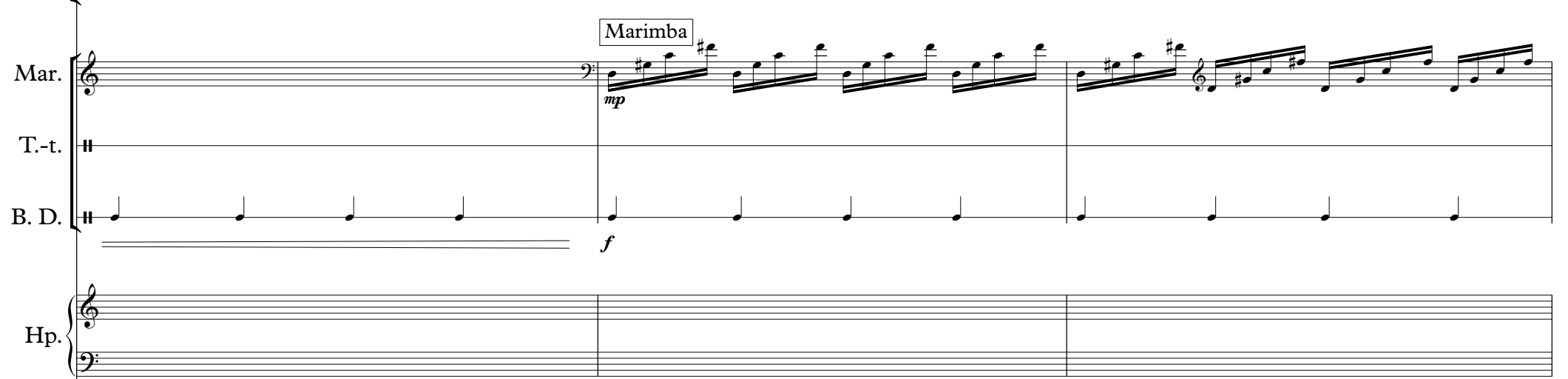

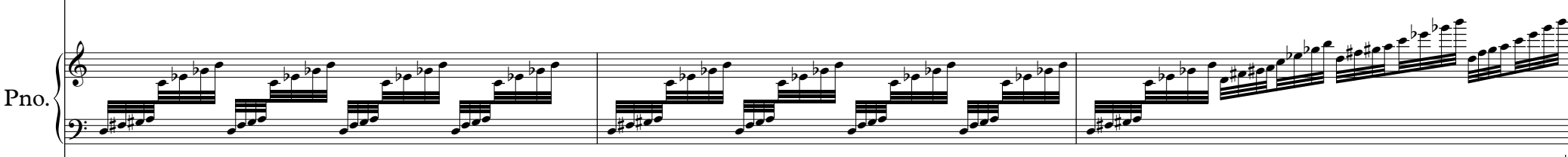

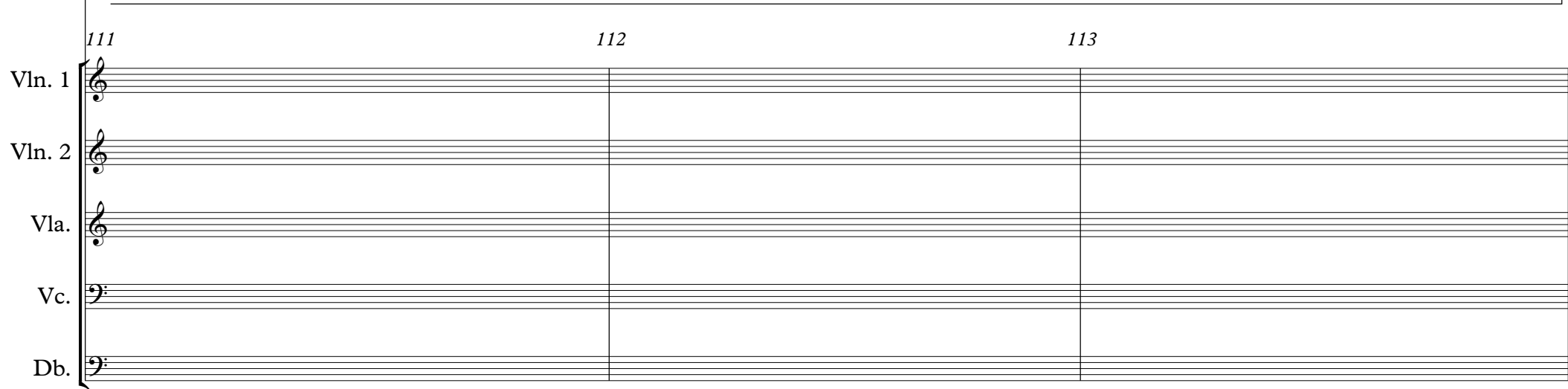


20
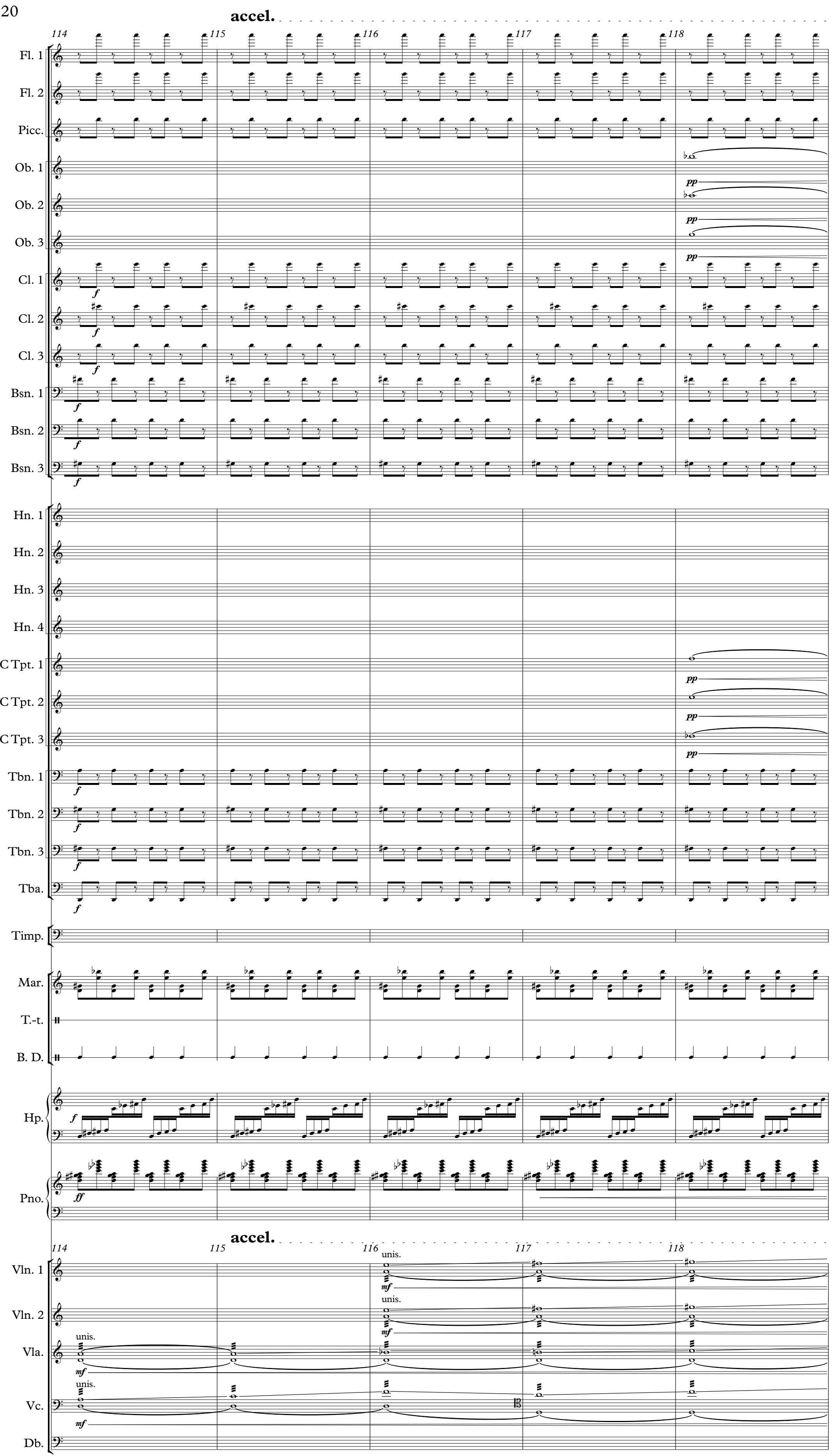

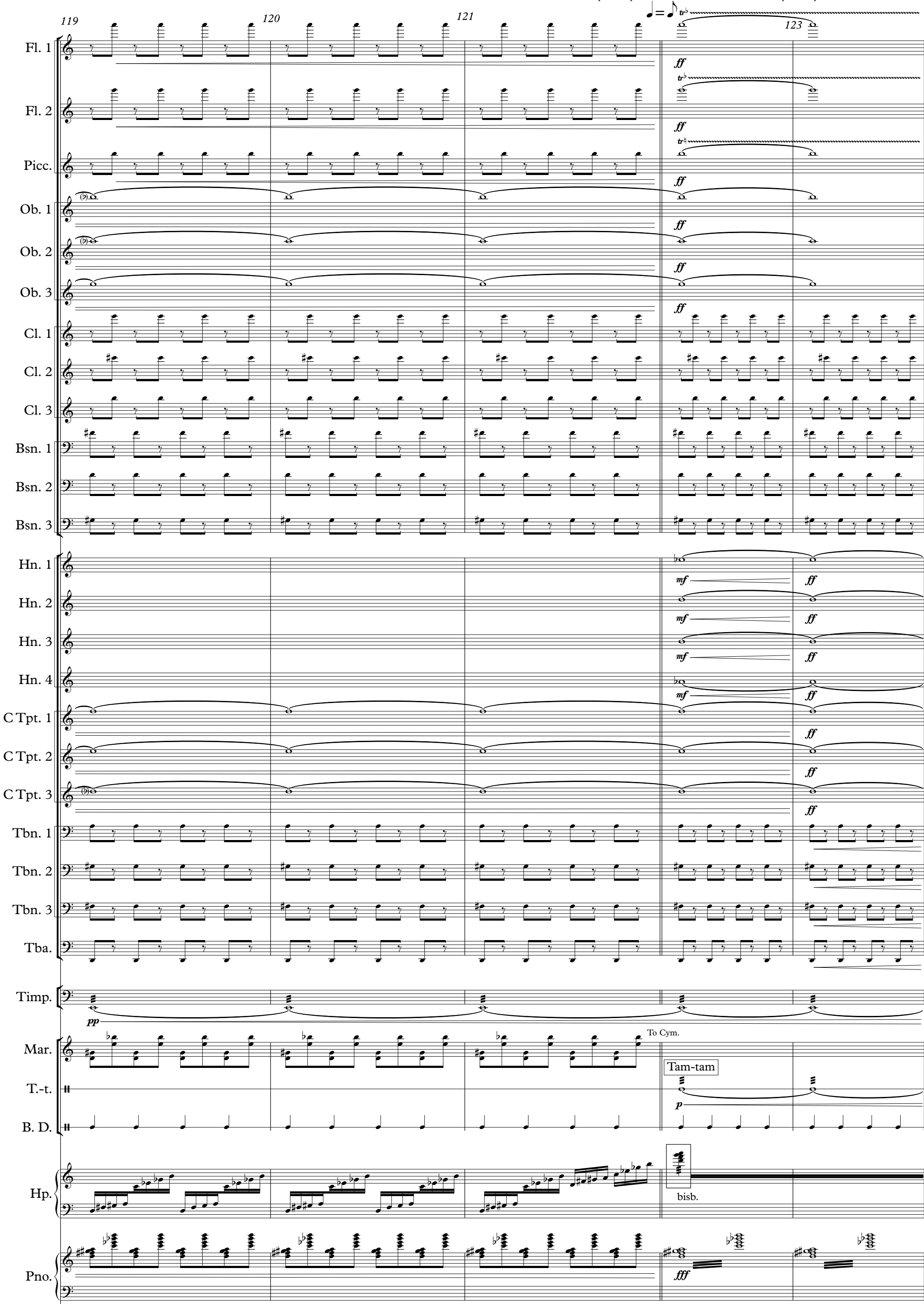

122 Half time $(.=100)$
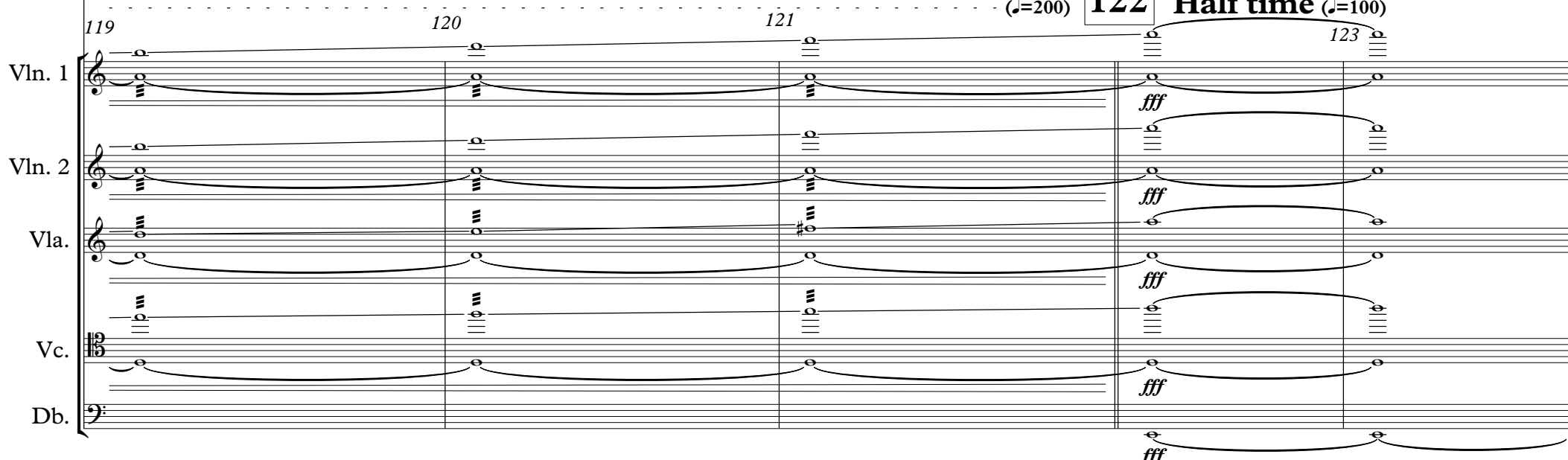

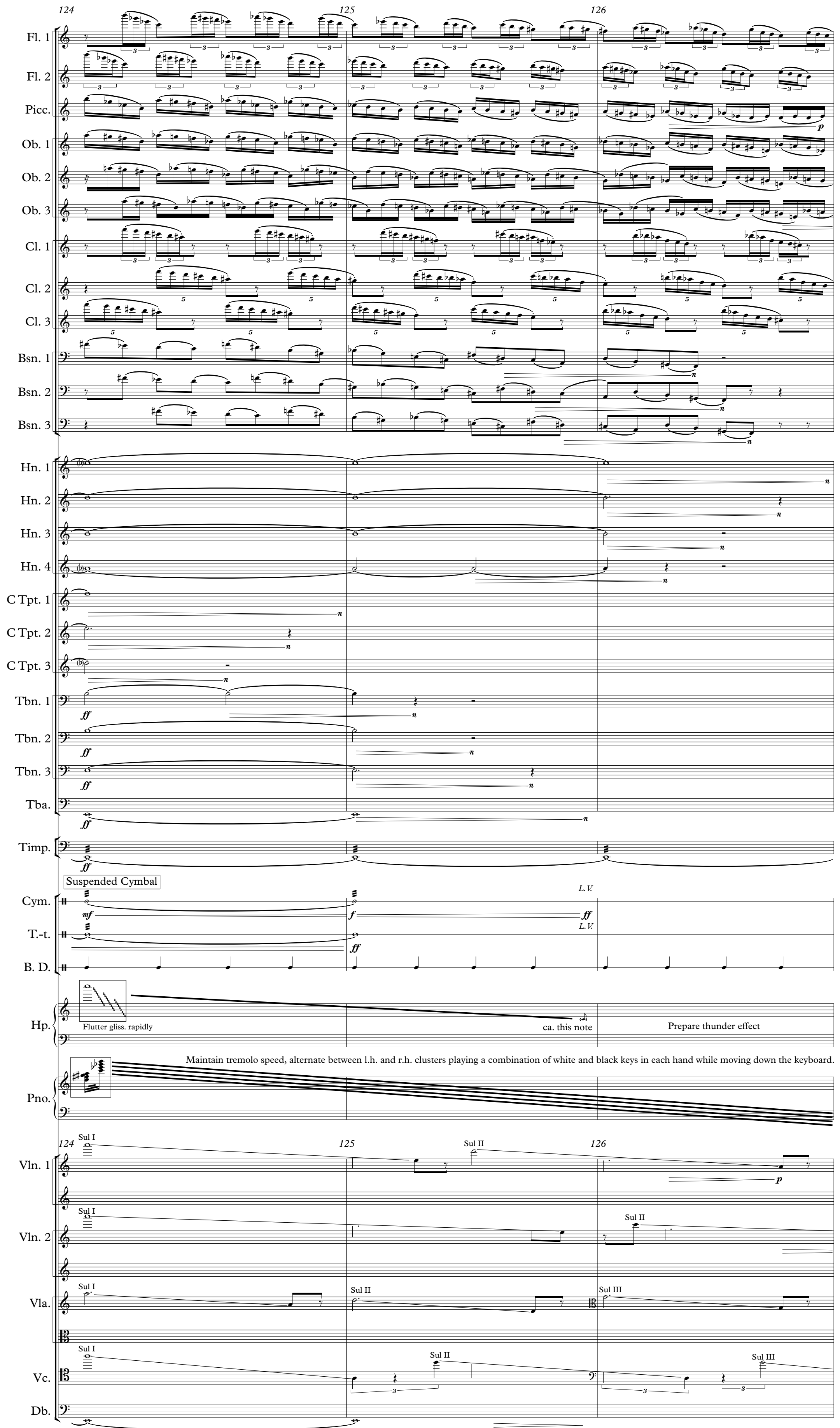

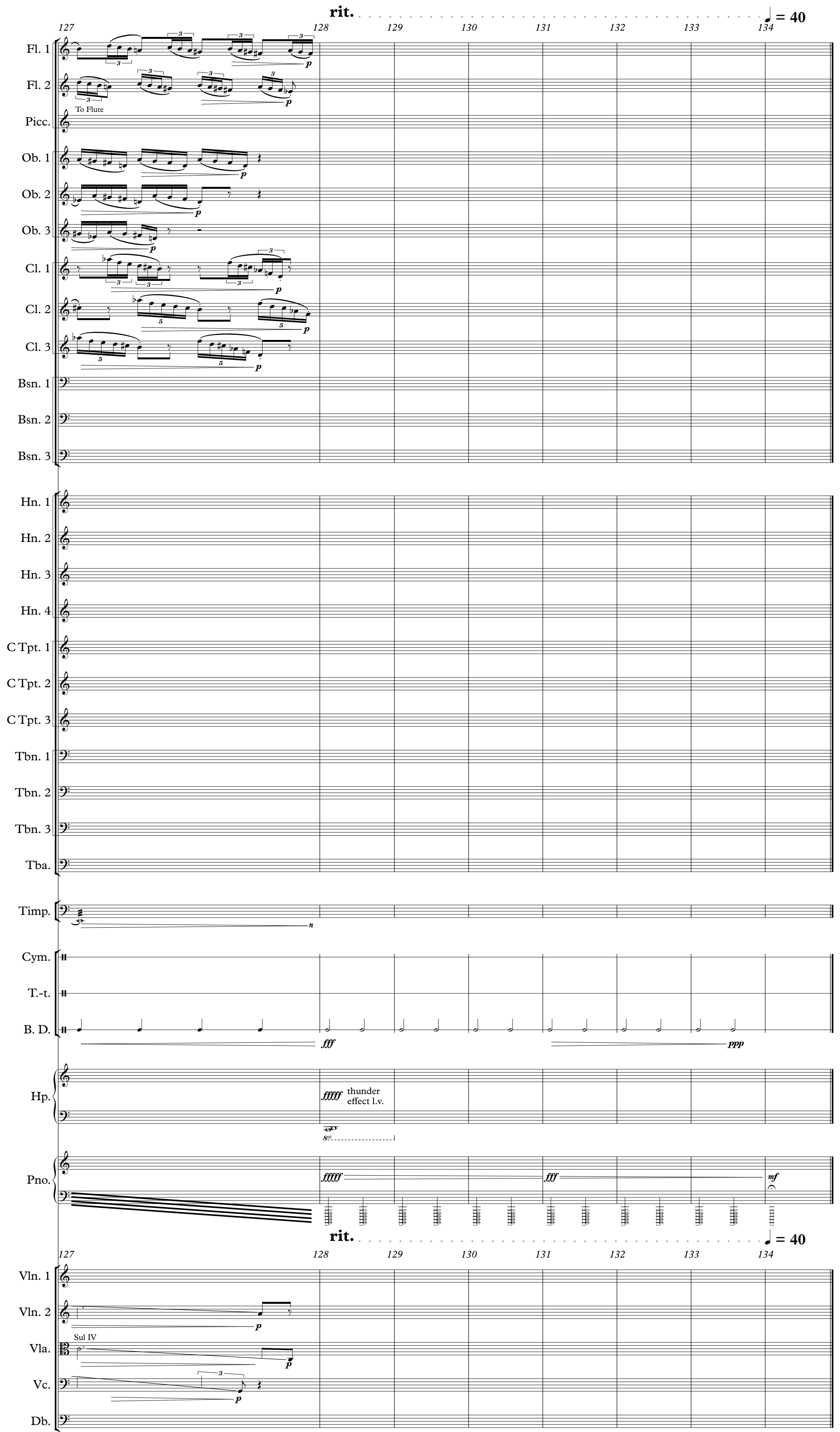

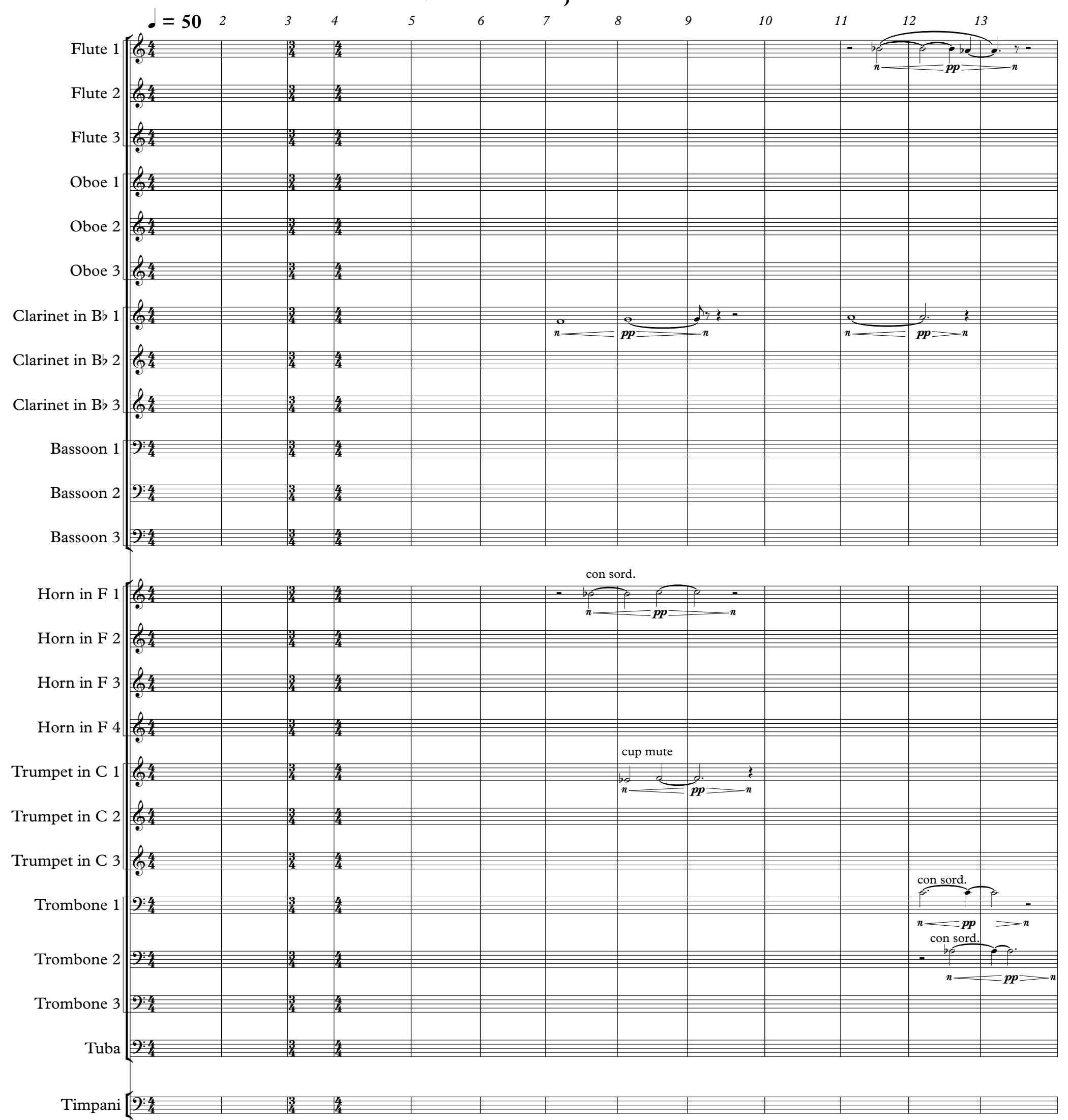

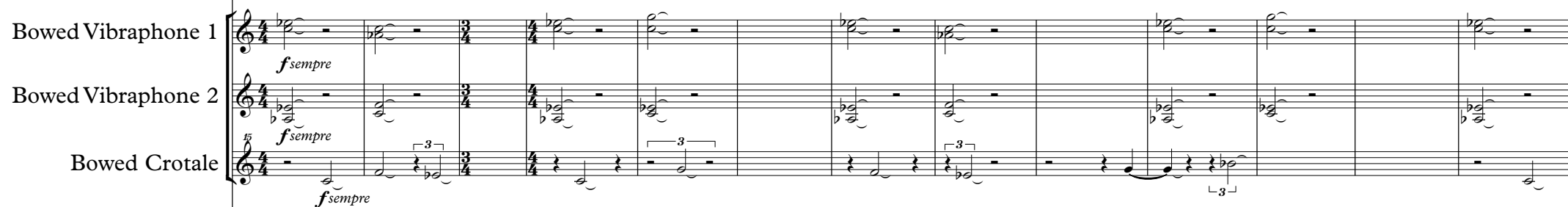

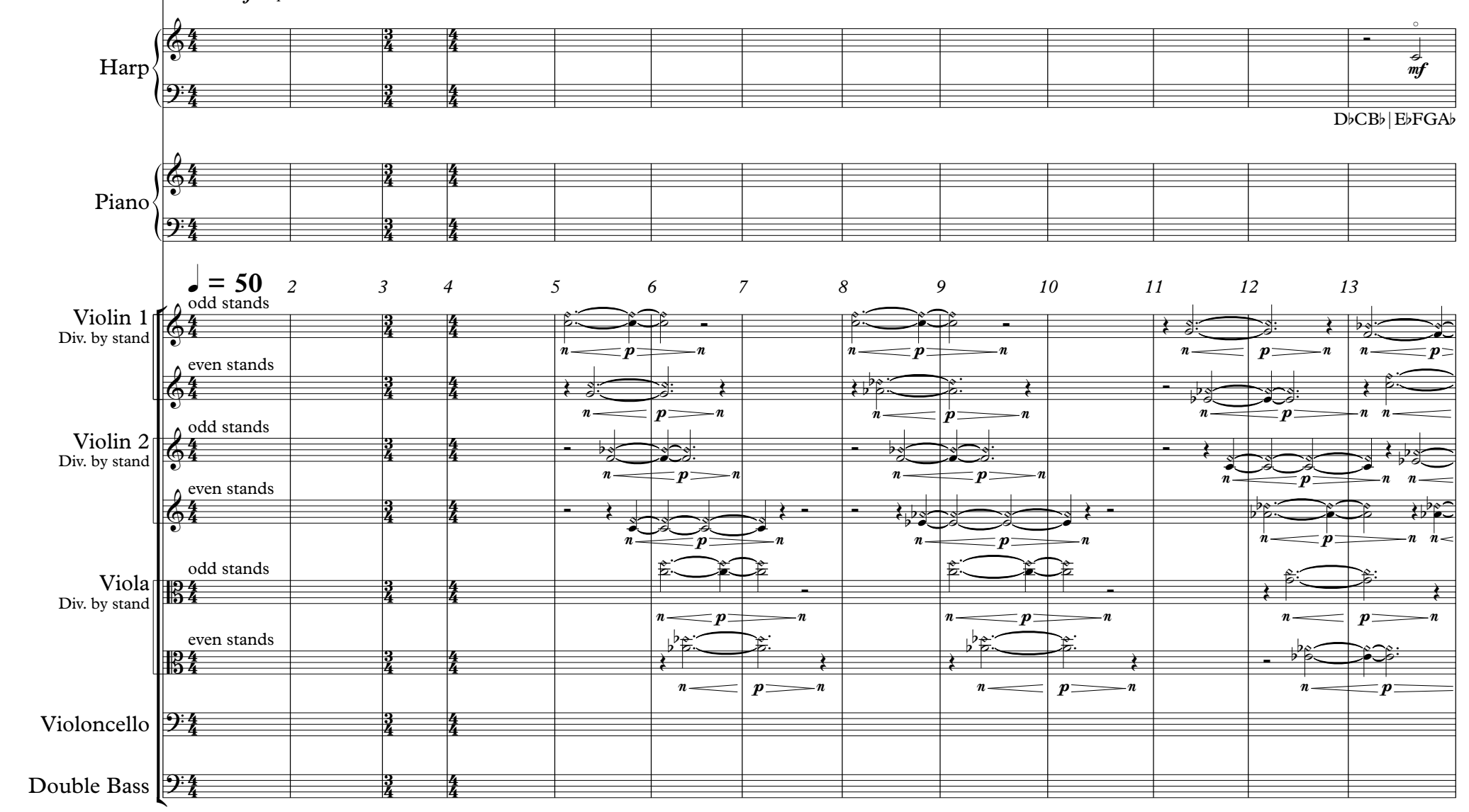


19

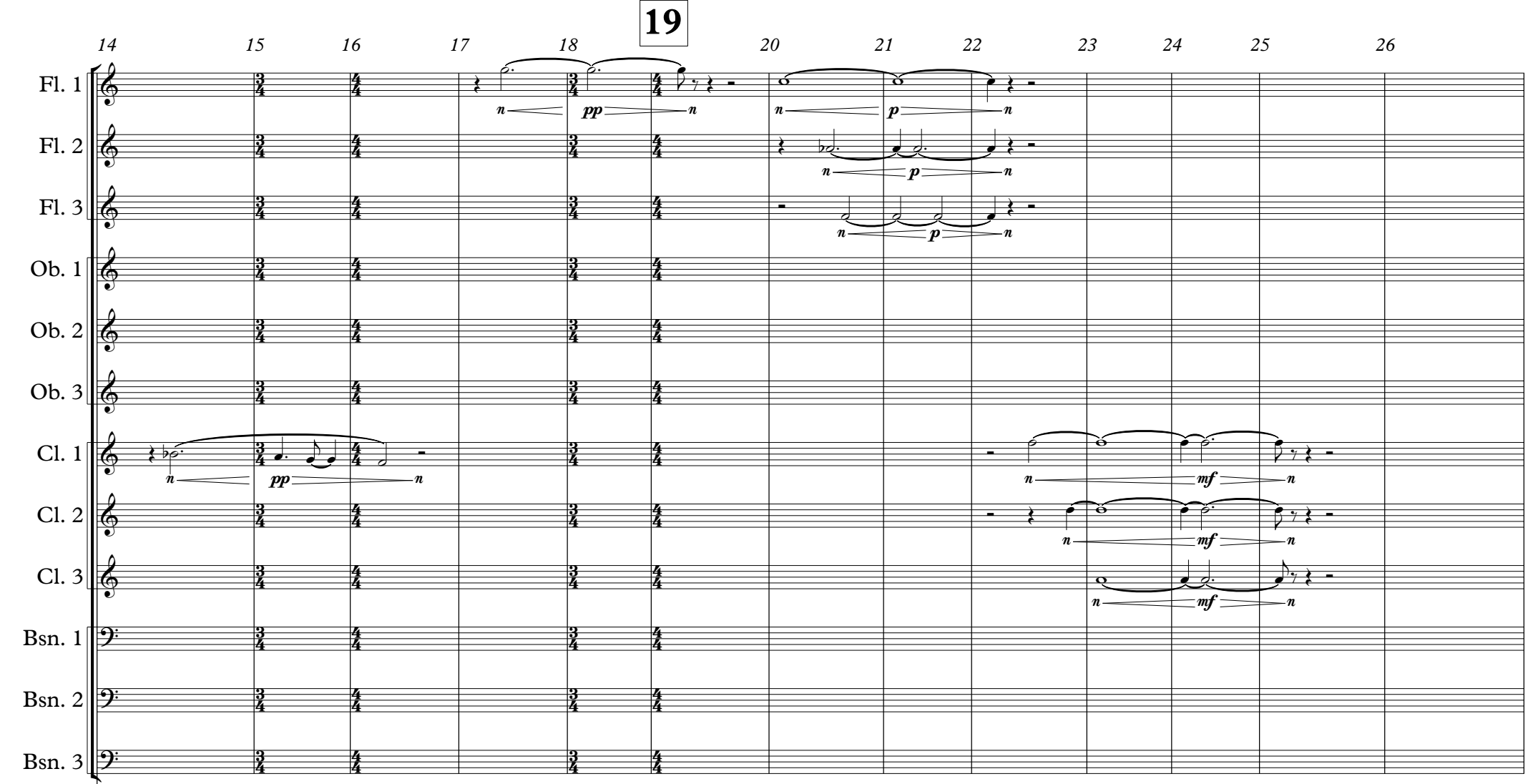

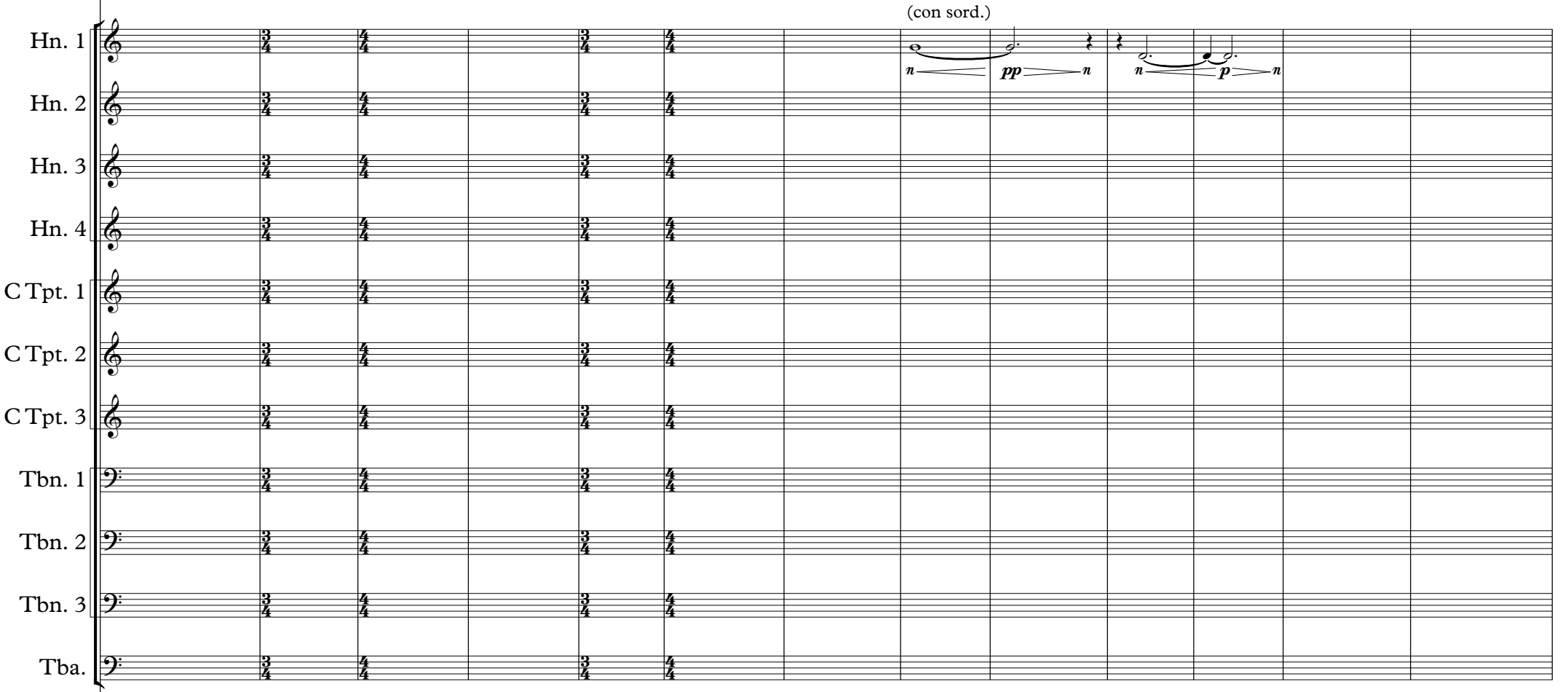

Timp.

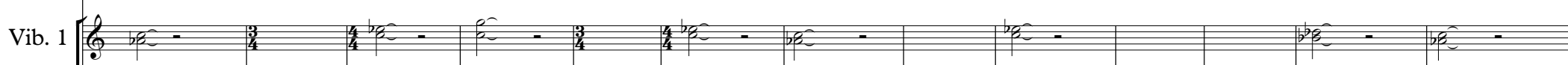

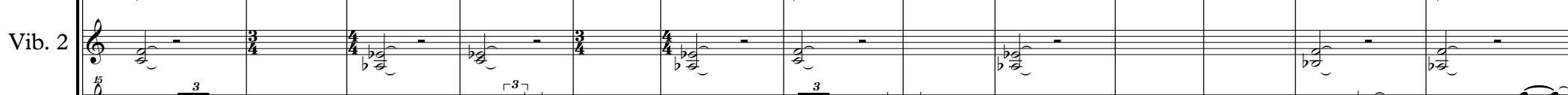

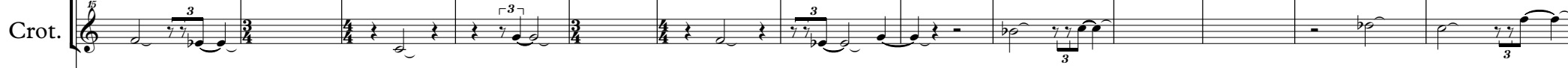

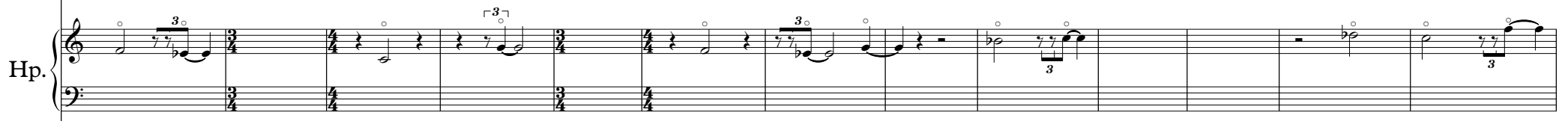

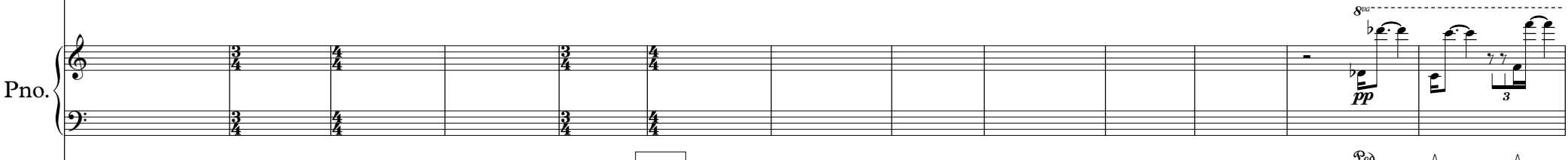

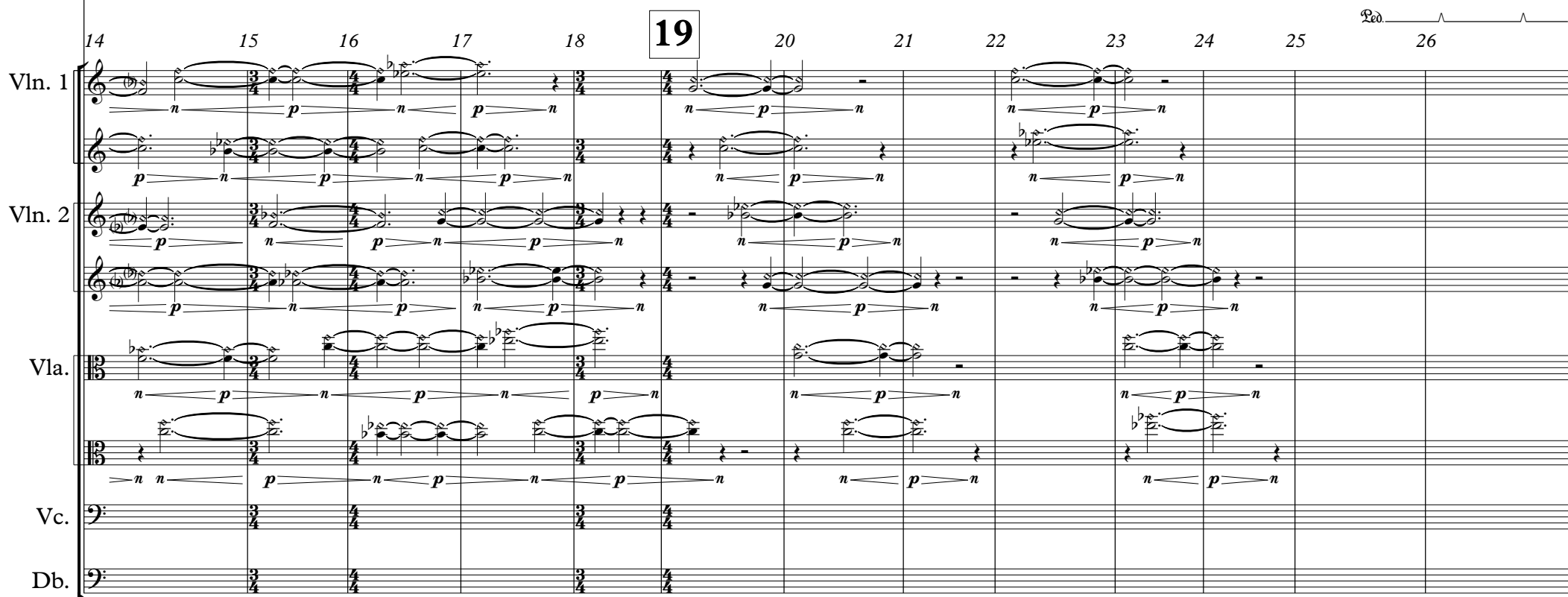




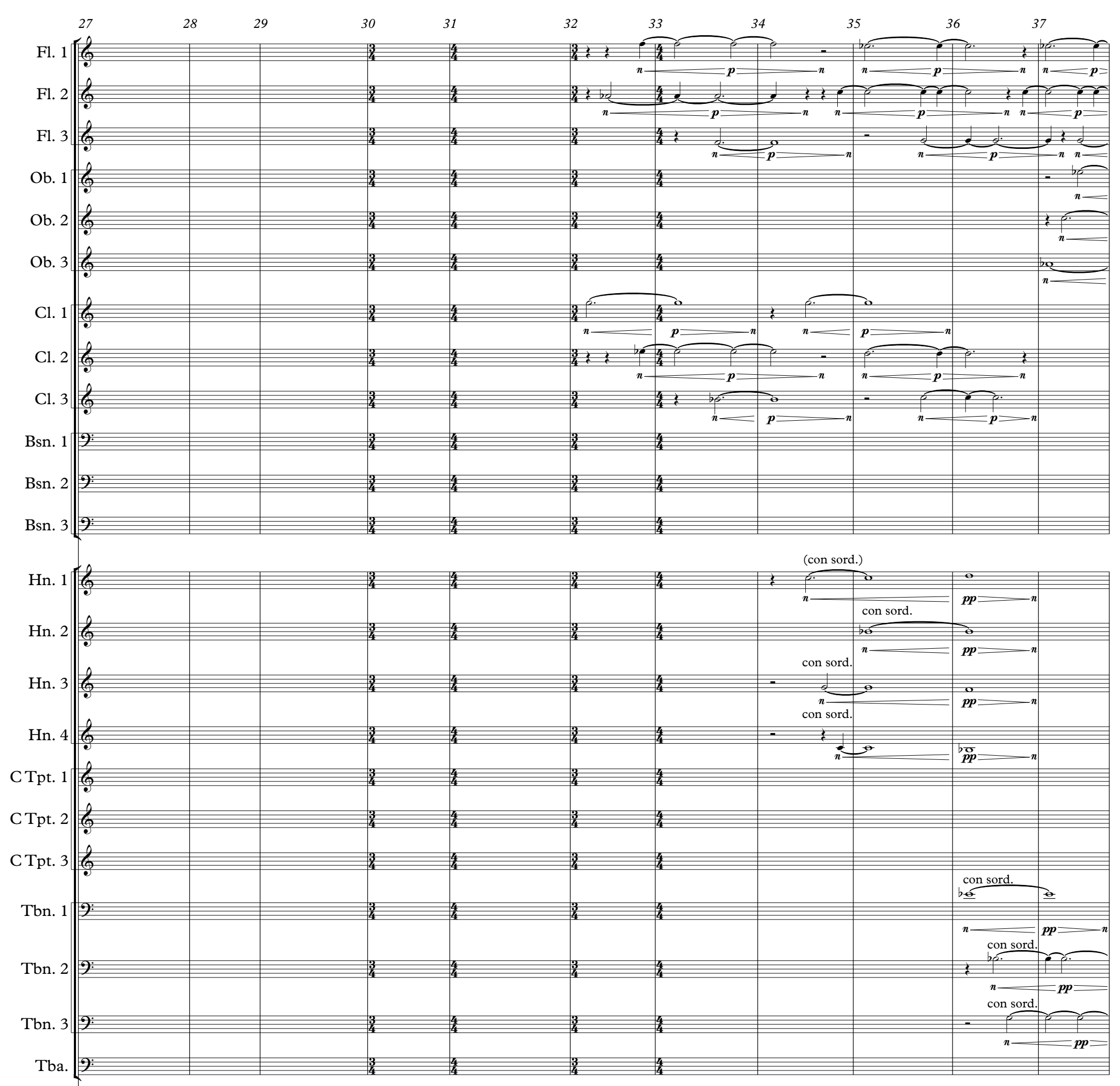

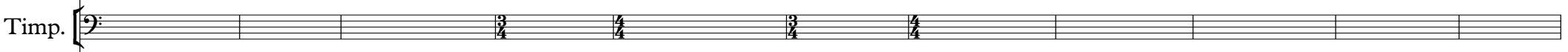

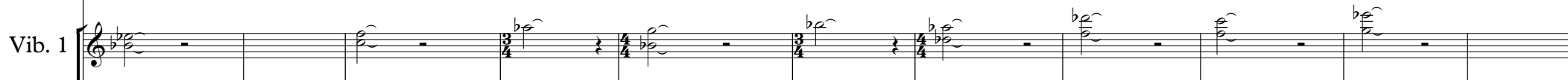

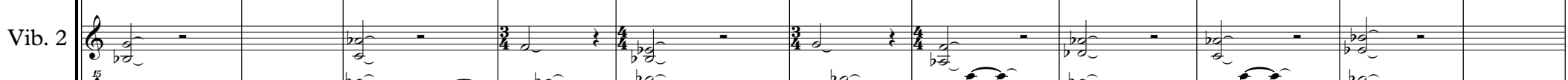

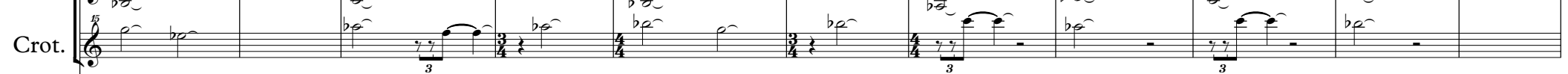

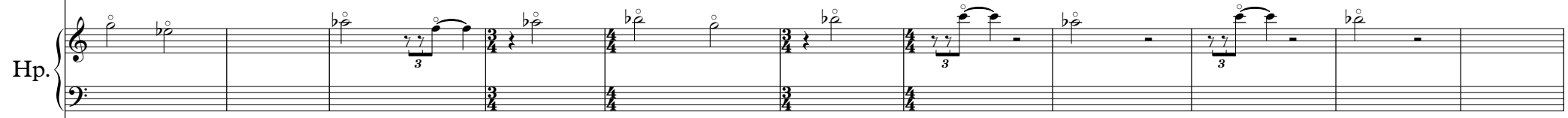

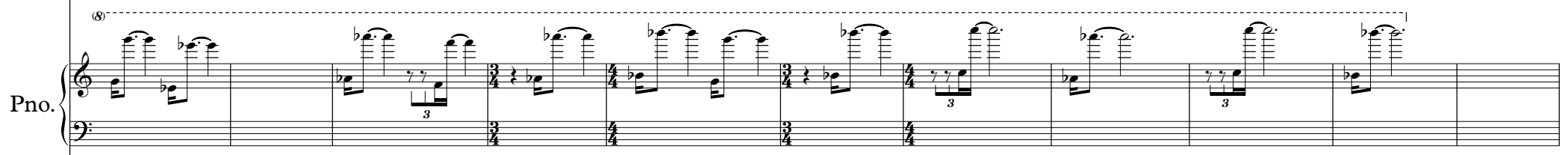

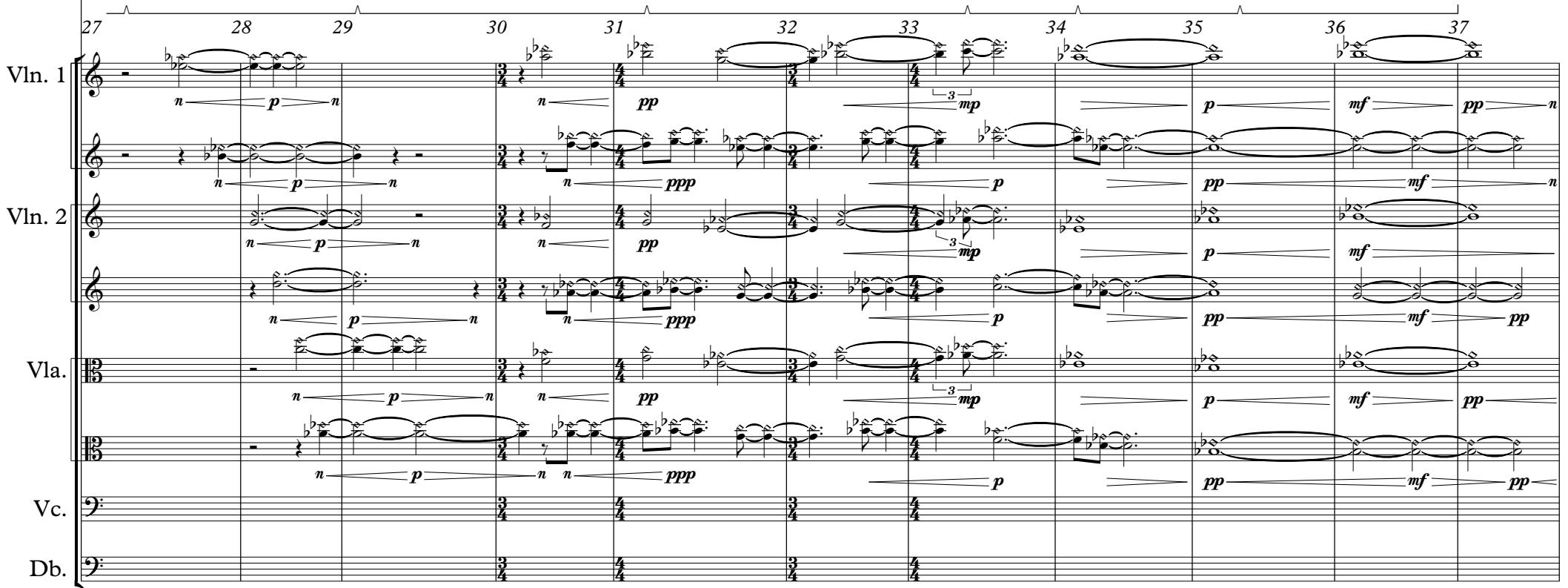


40

$\begin{array}{lll}41 & 42 & 43\end{array}$
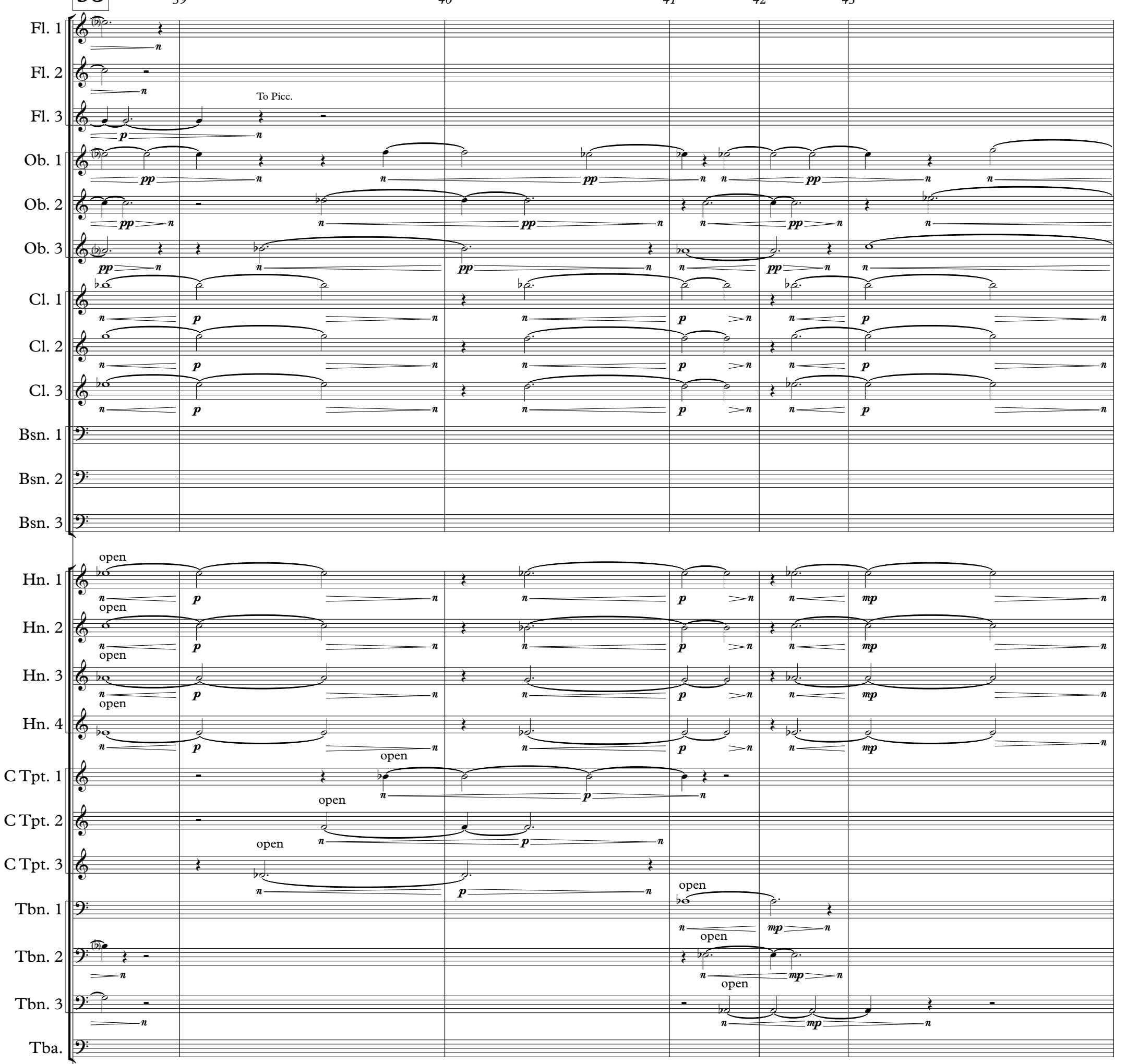

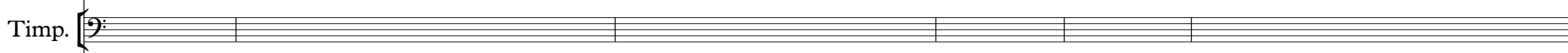

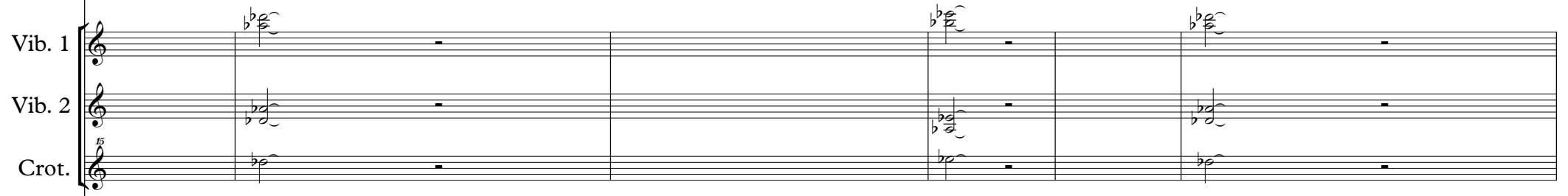

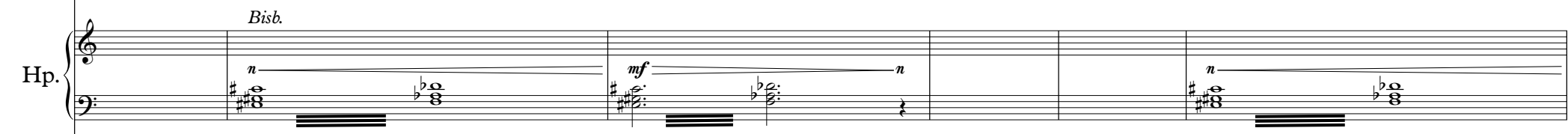

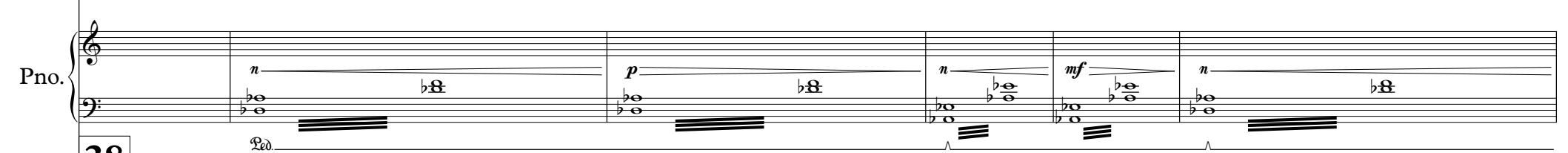

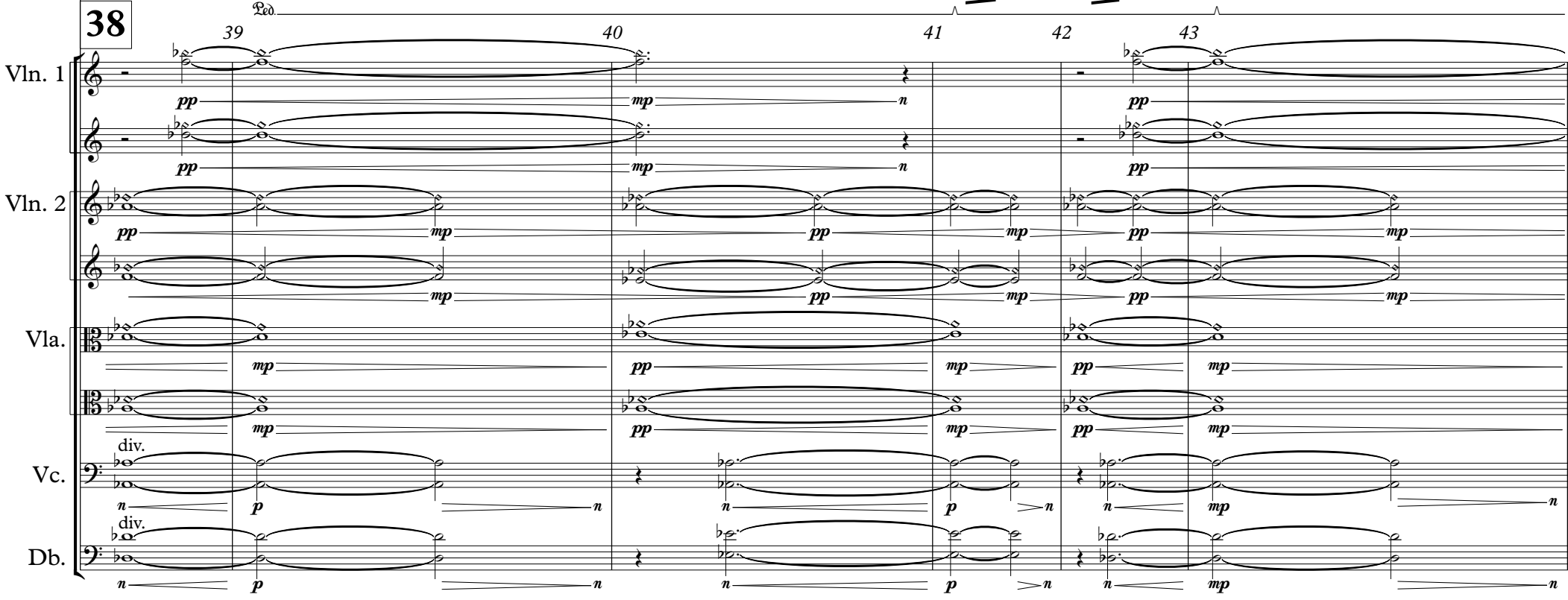



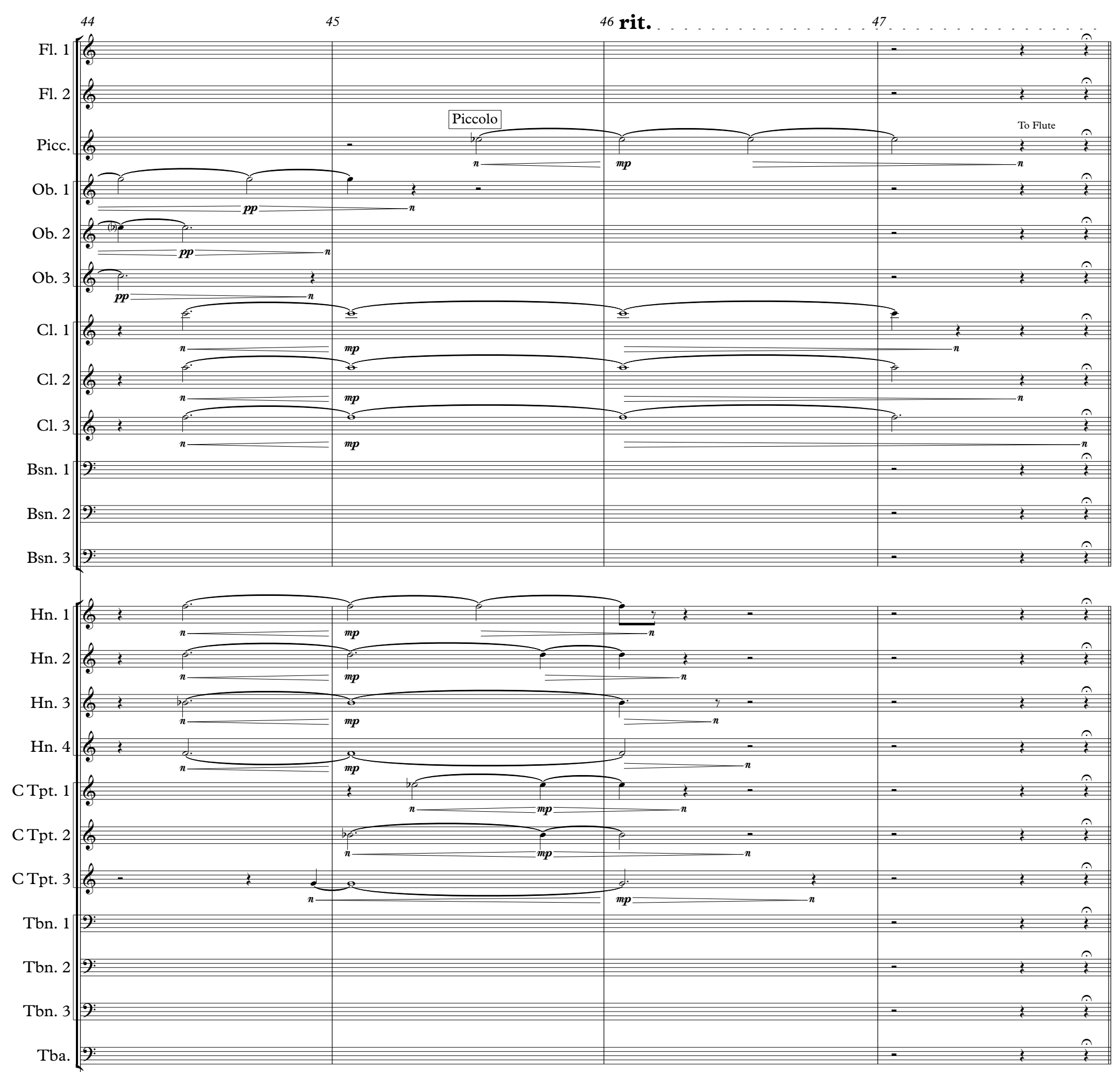

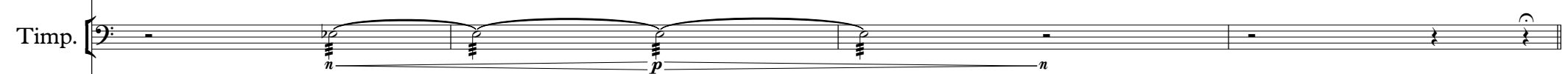
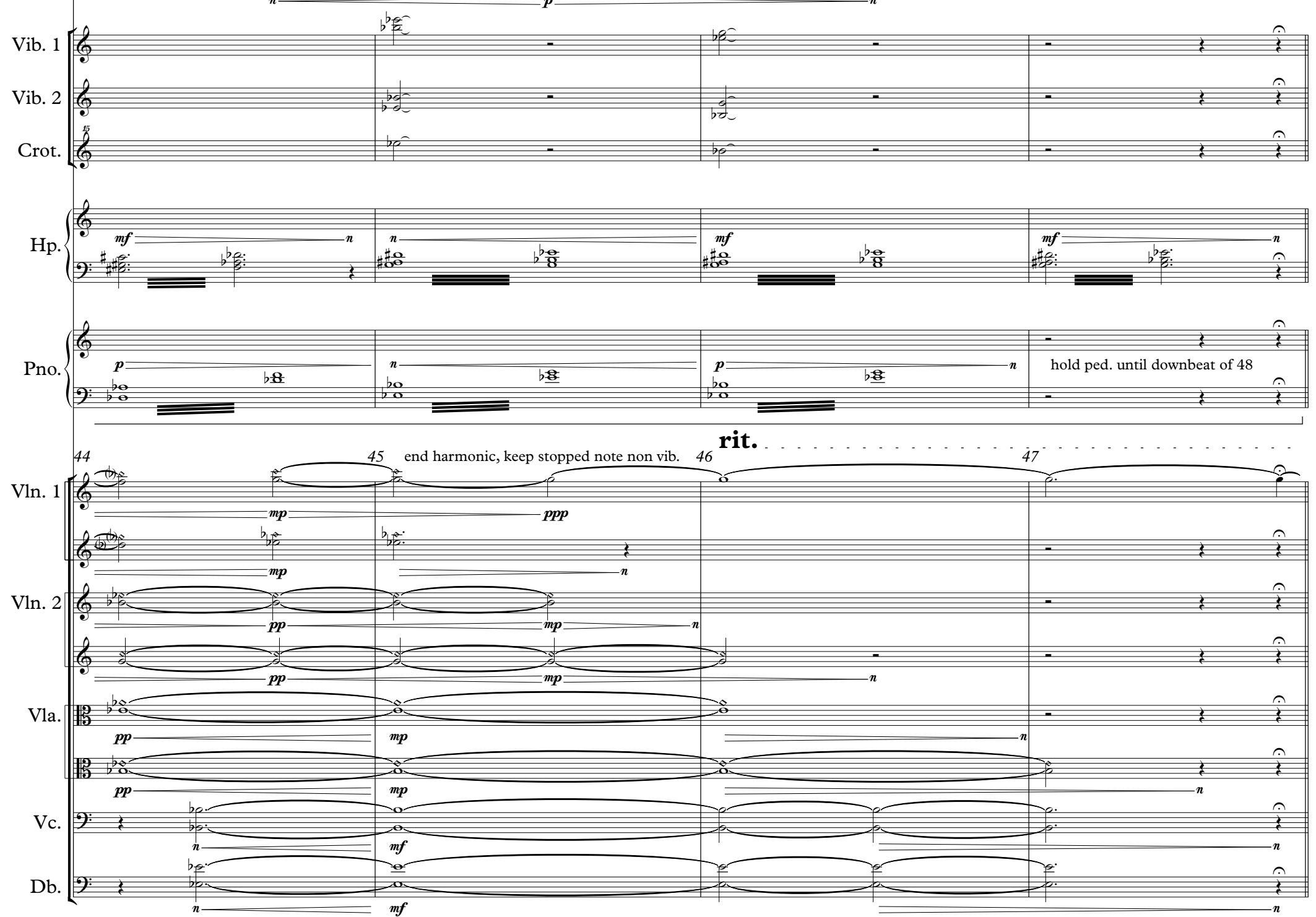
48 a tempo $(\cdot=50)$

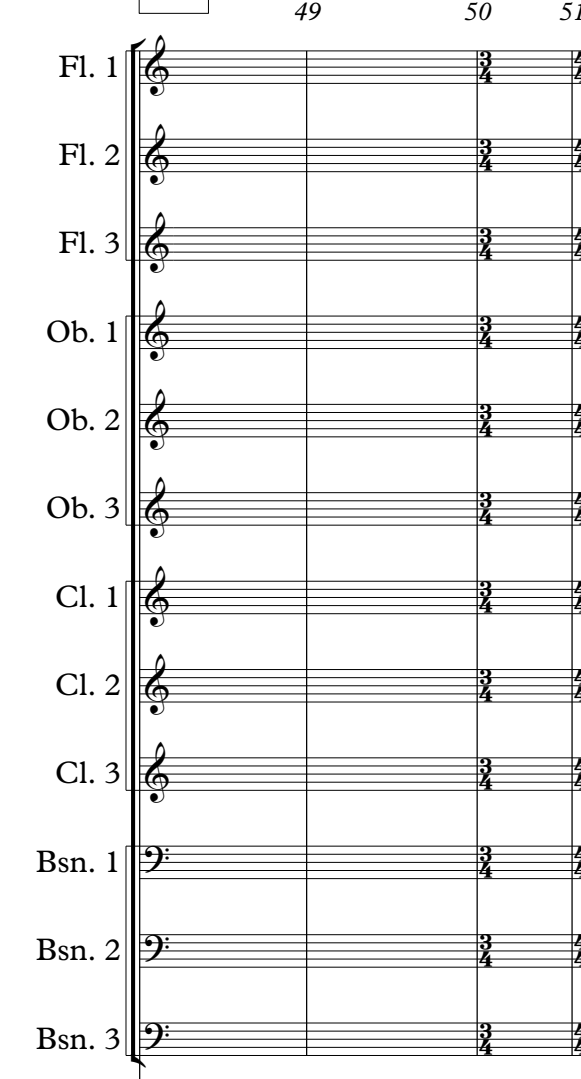

$52-53-54=54$
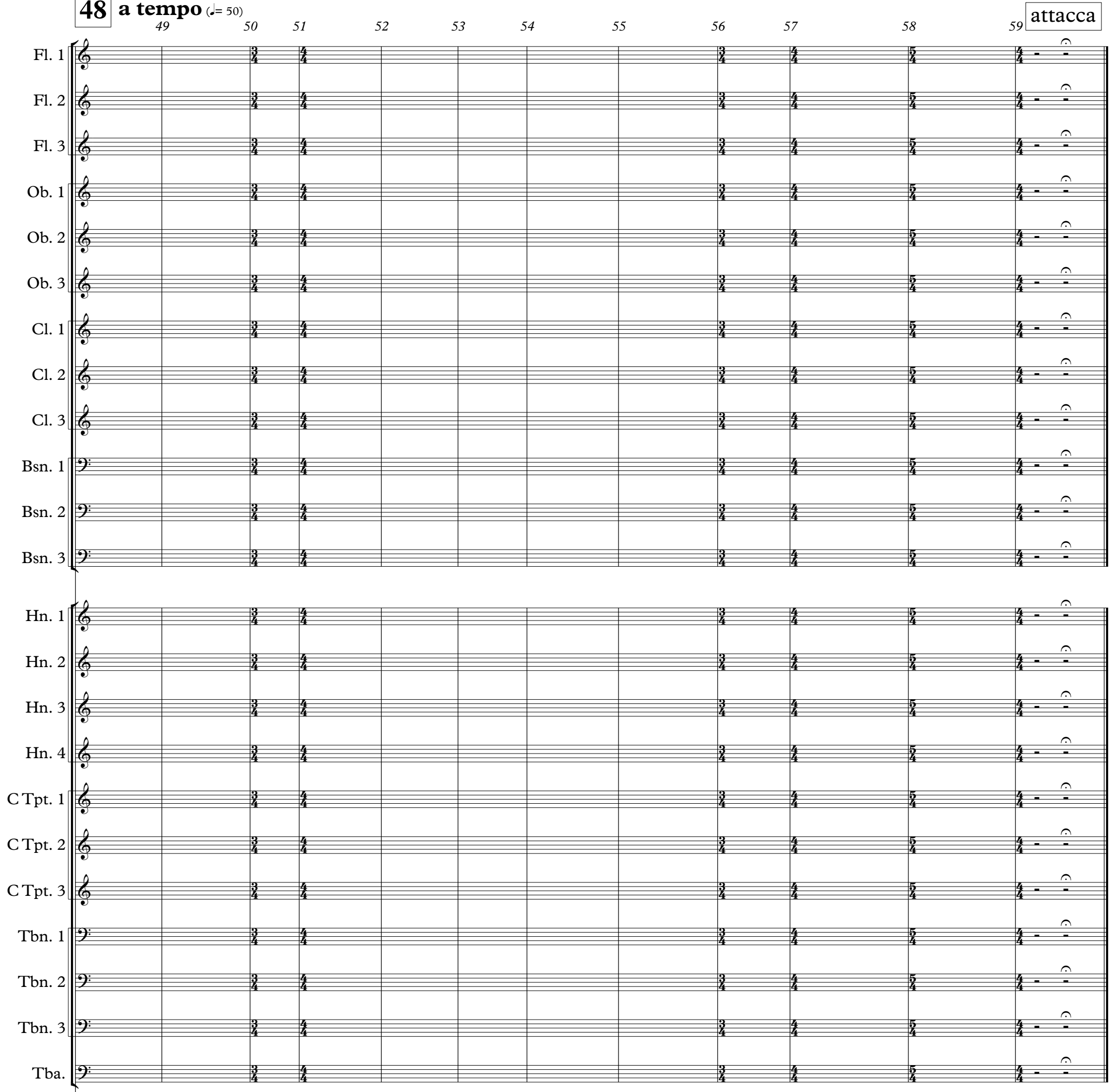

Timp. 㑌

$\frac{3}{4}$

?

$4_{4}^{15}$

$\stackrel{0}{20}$

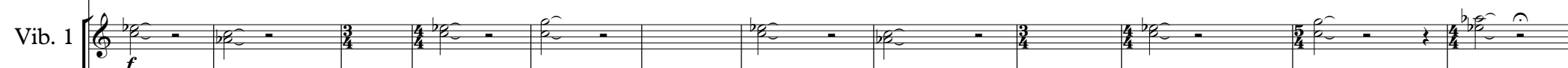

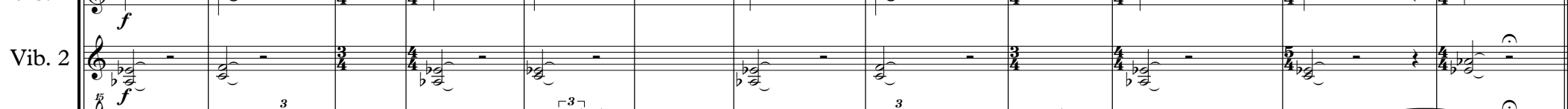

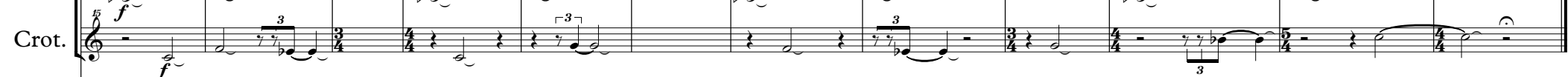

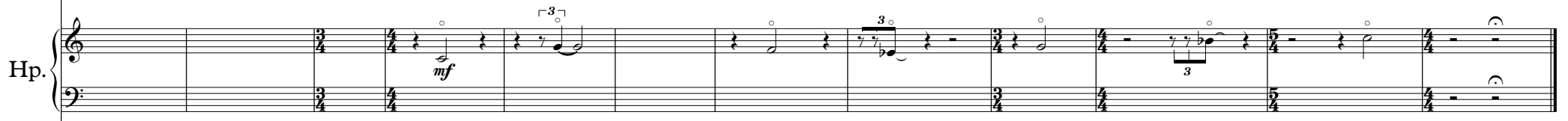

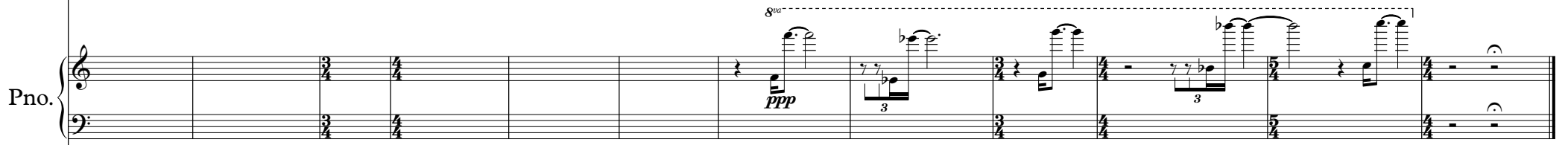
48 a tempo $(\cdot=50)$

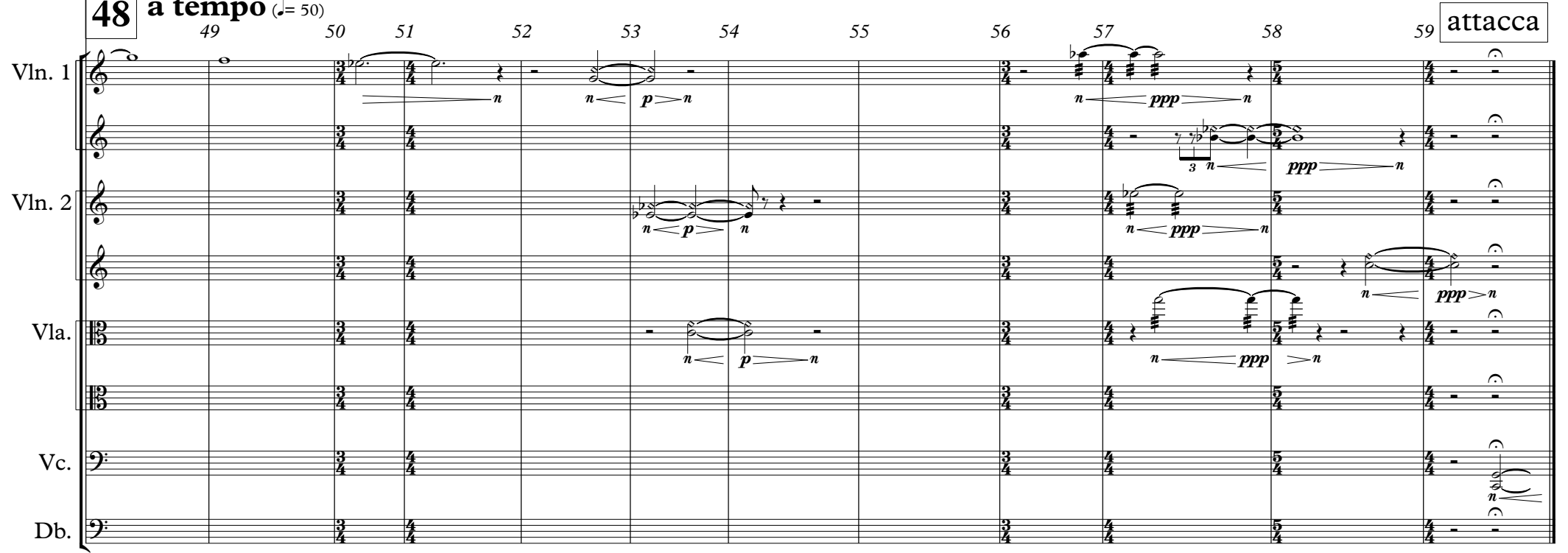


.$=56$ anxious, expectant.
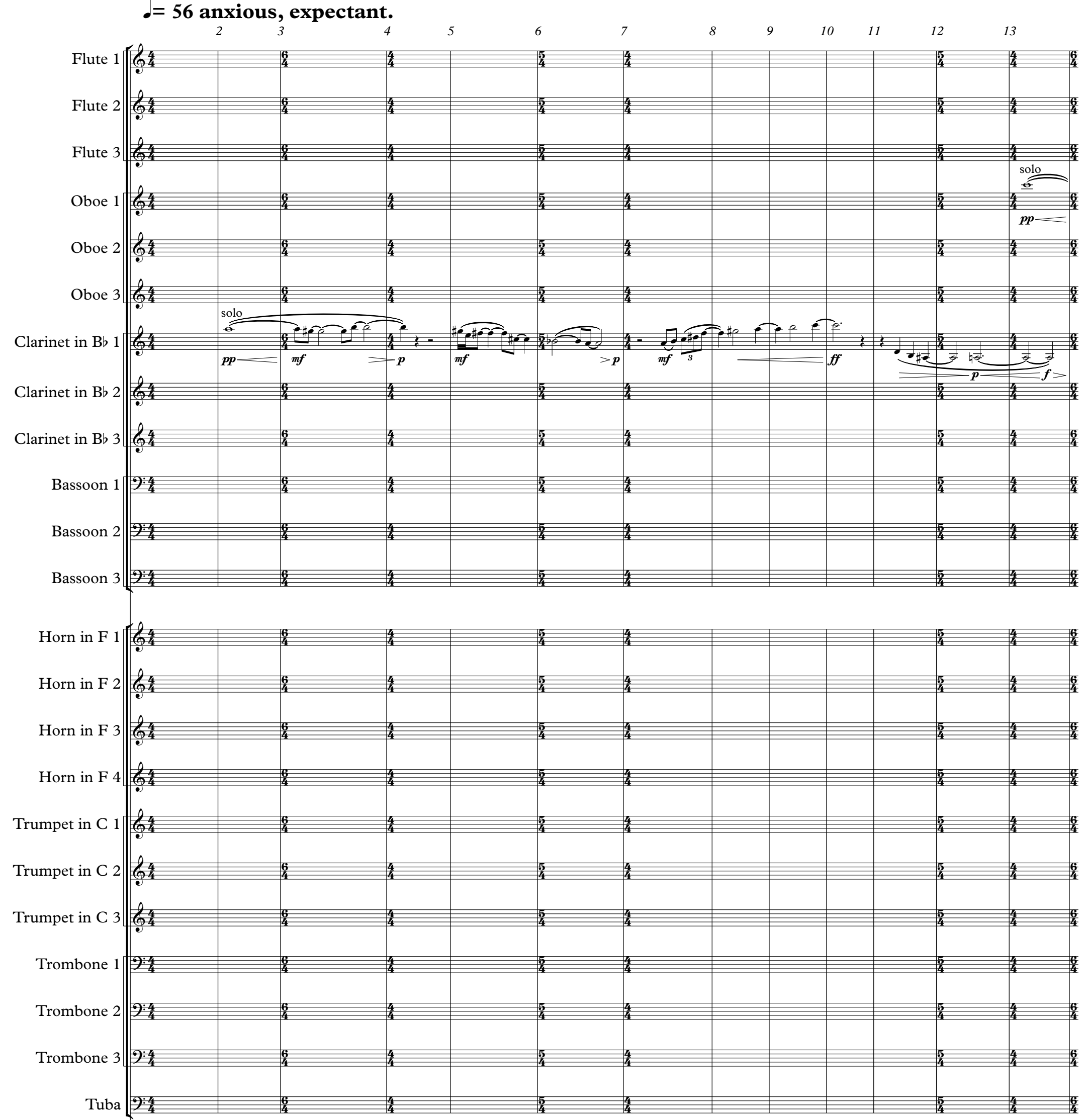

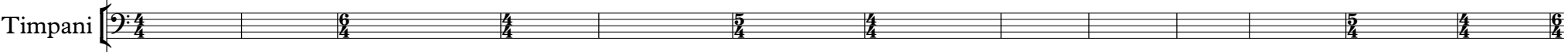
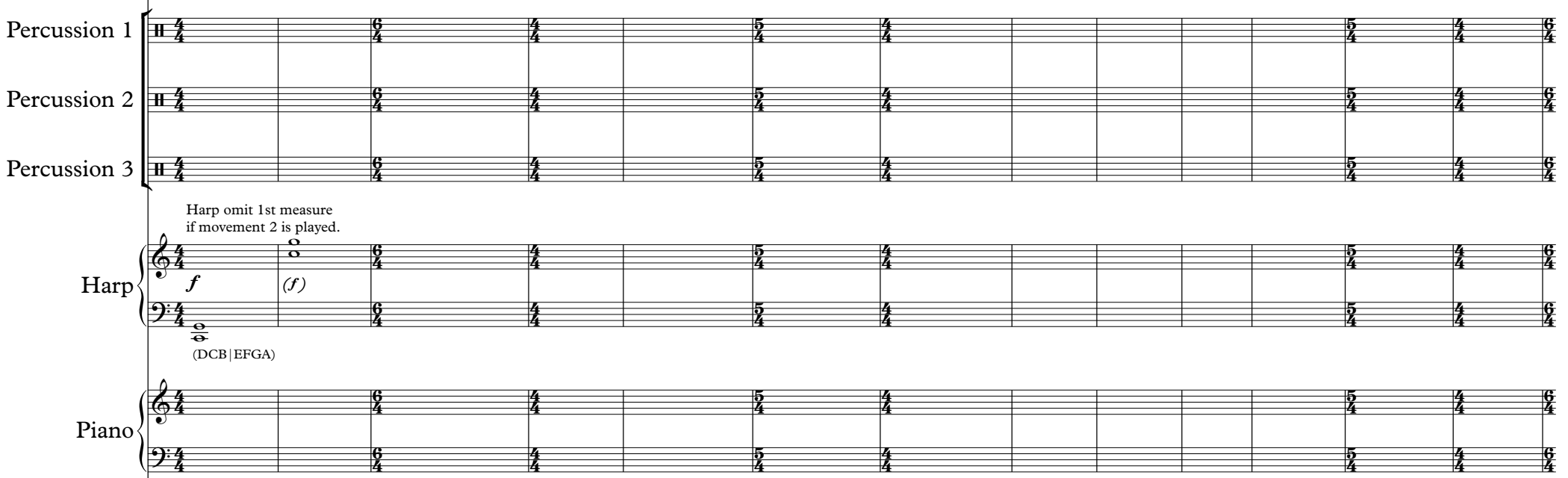

$\cdot=56$ anxious, expectant.

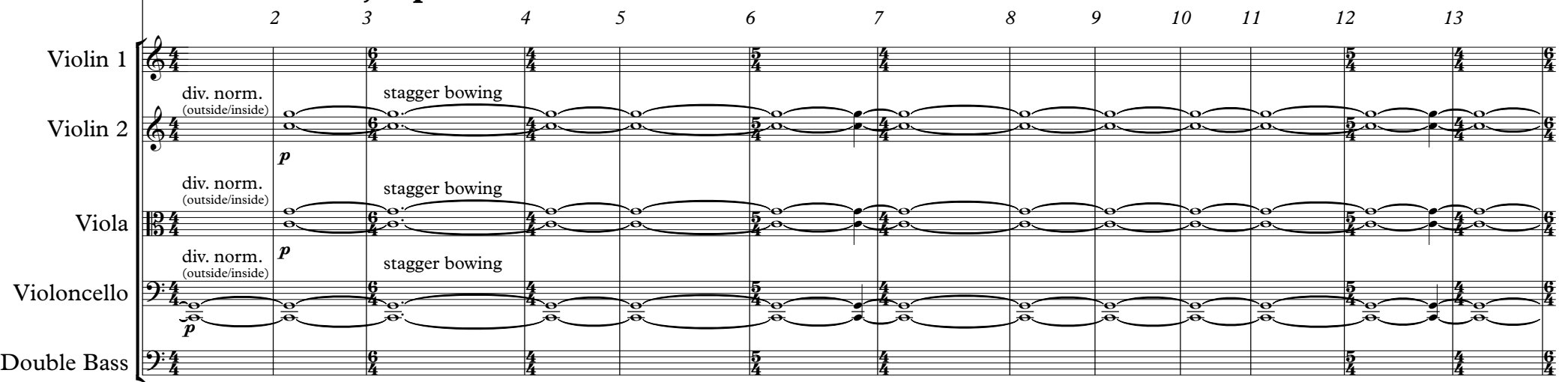



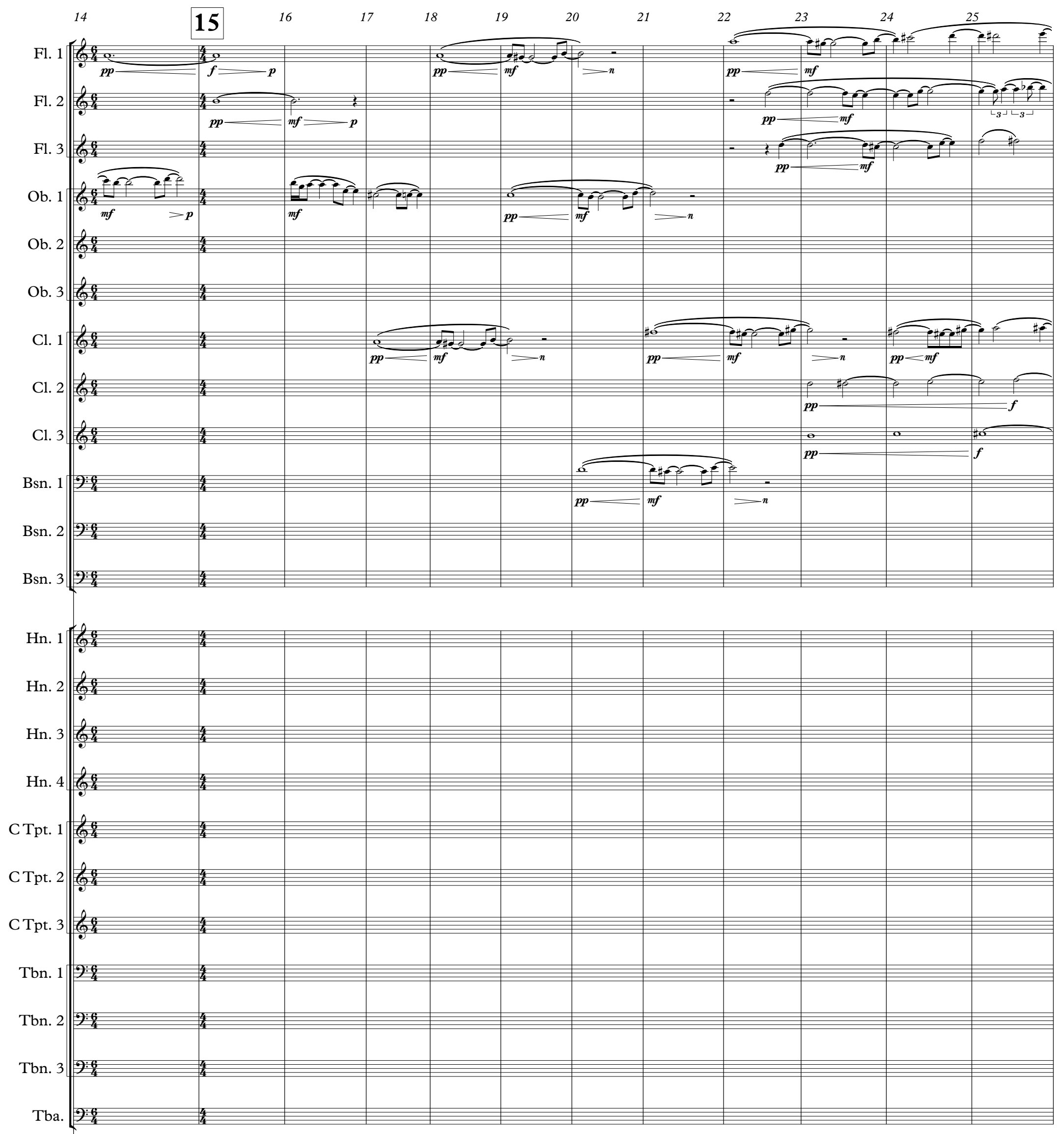

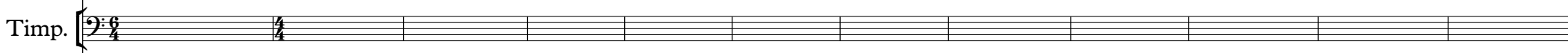
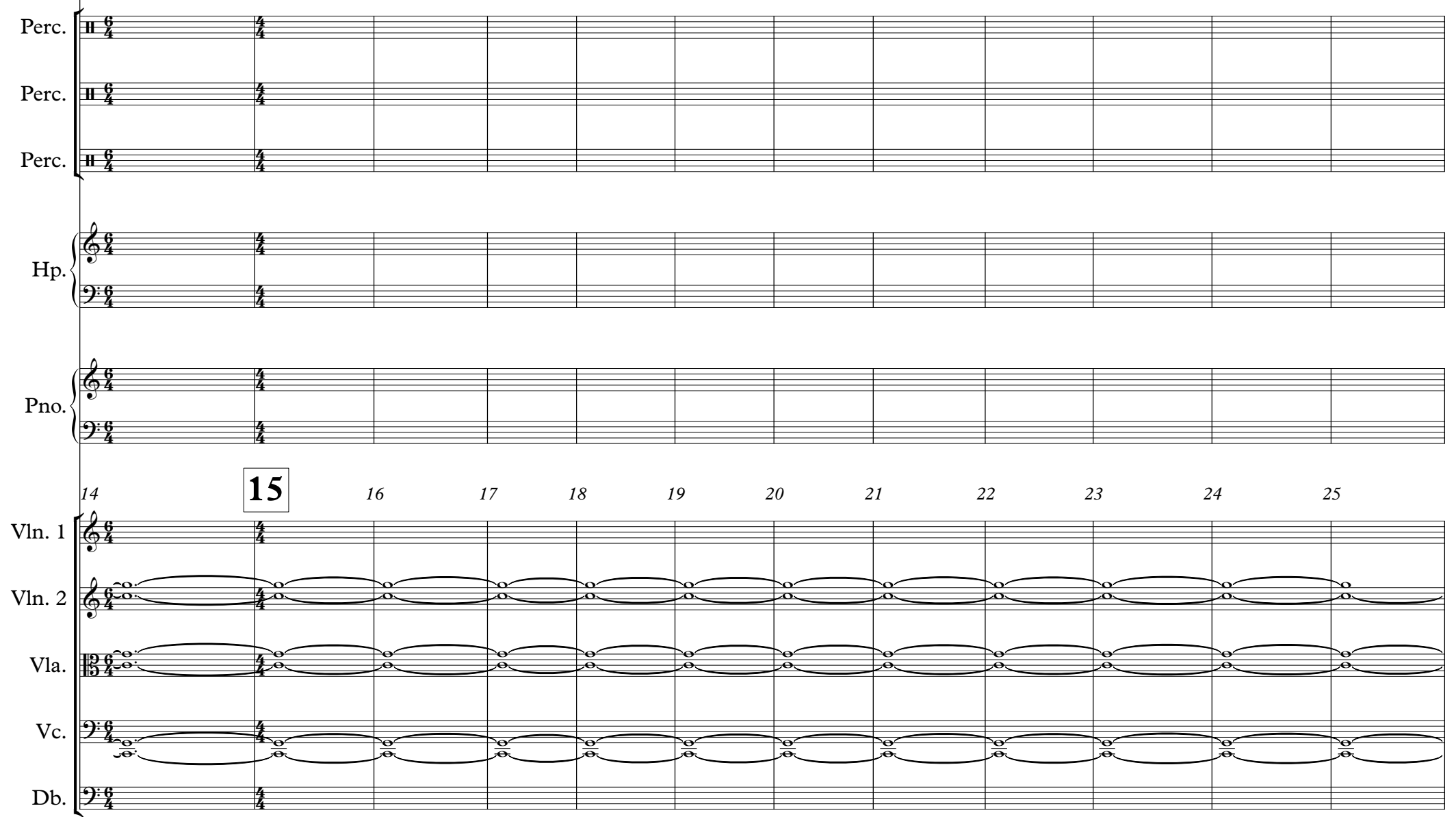

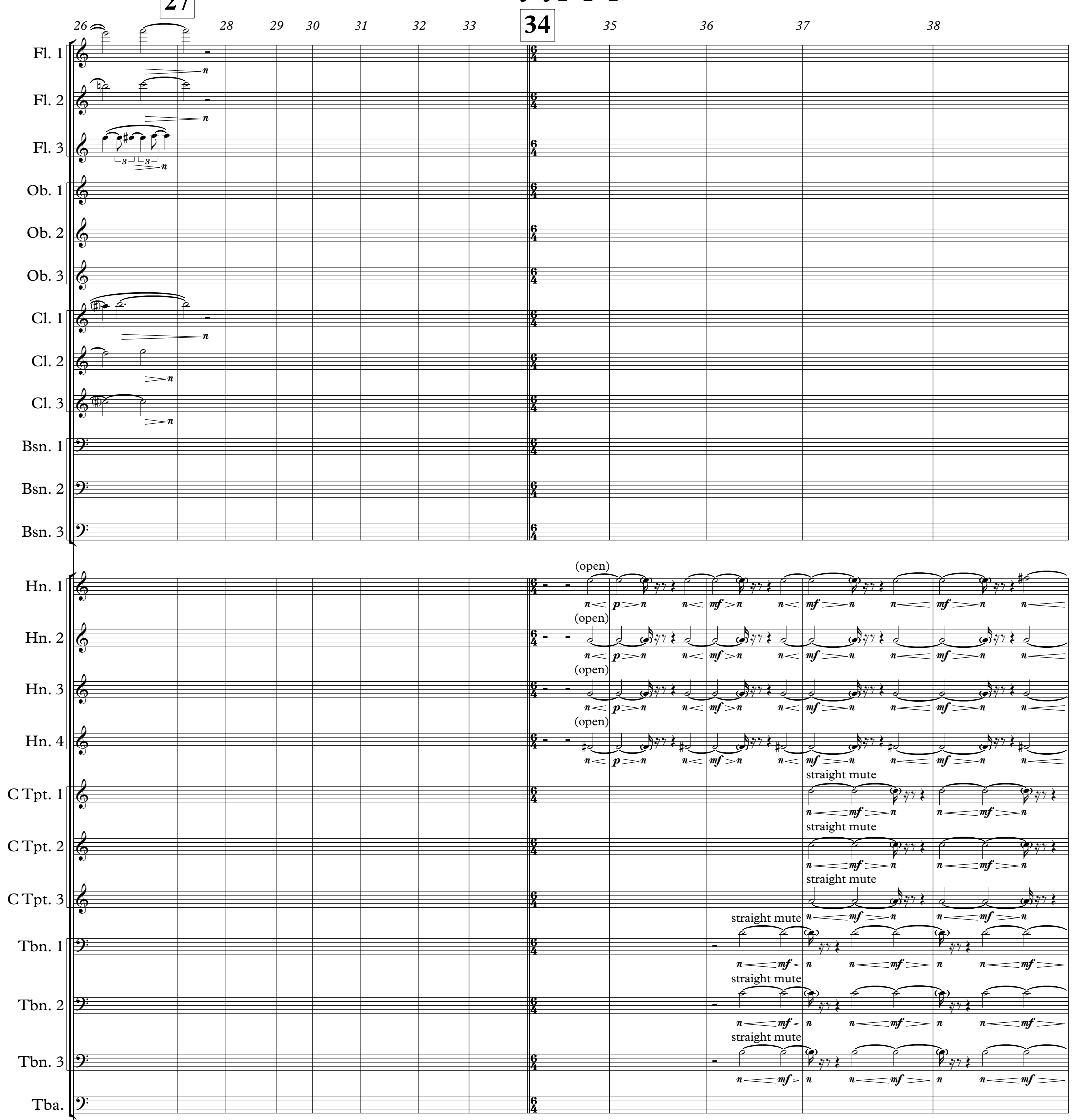

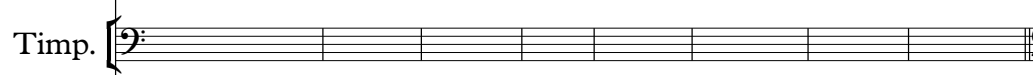

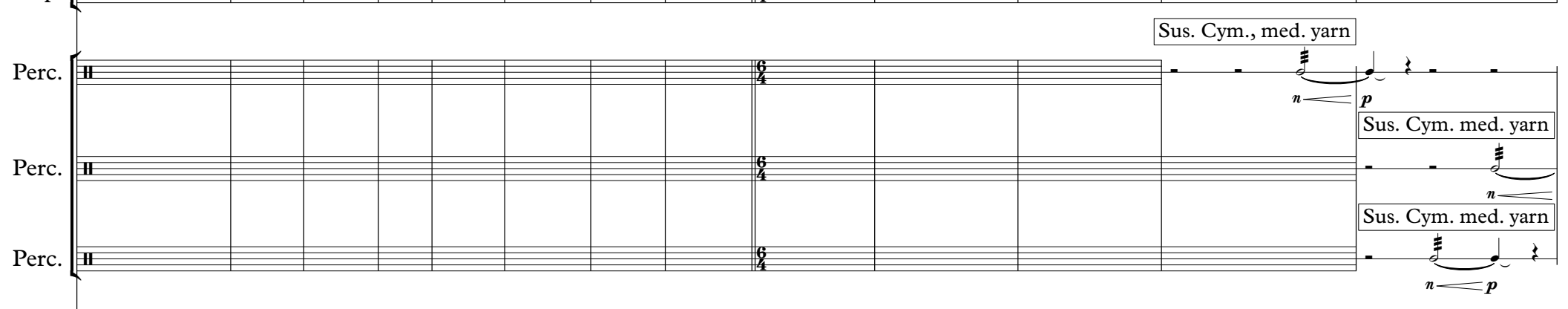

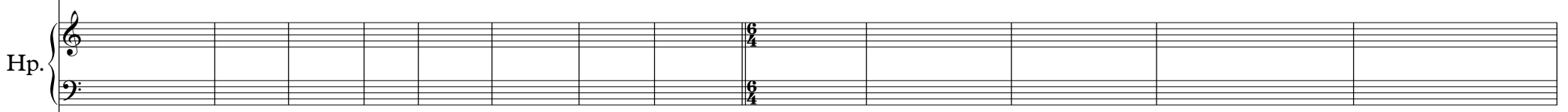
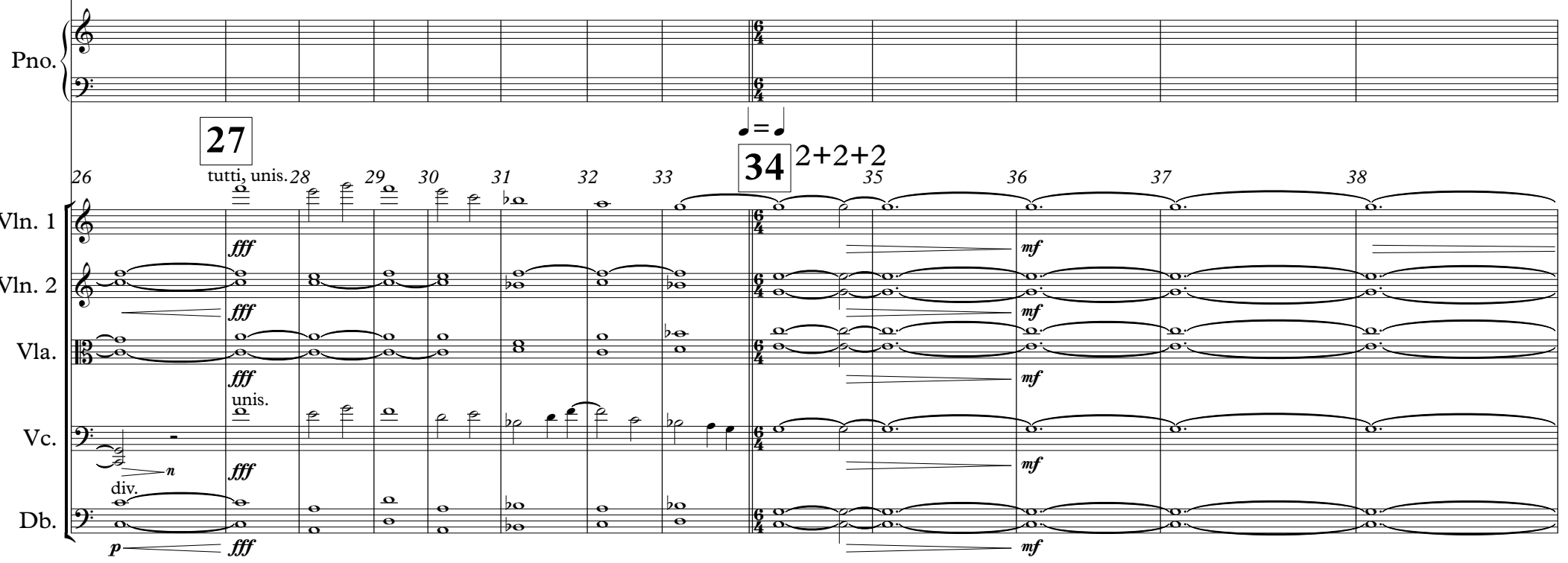

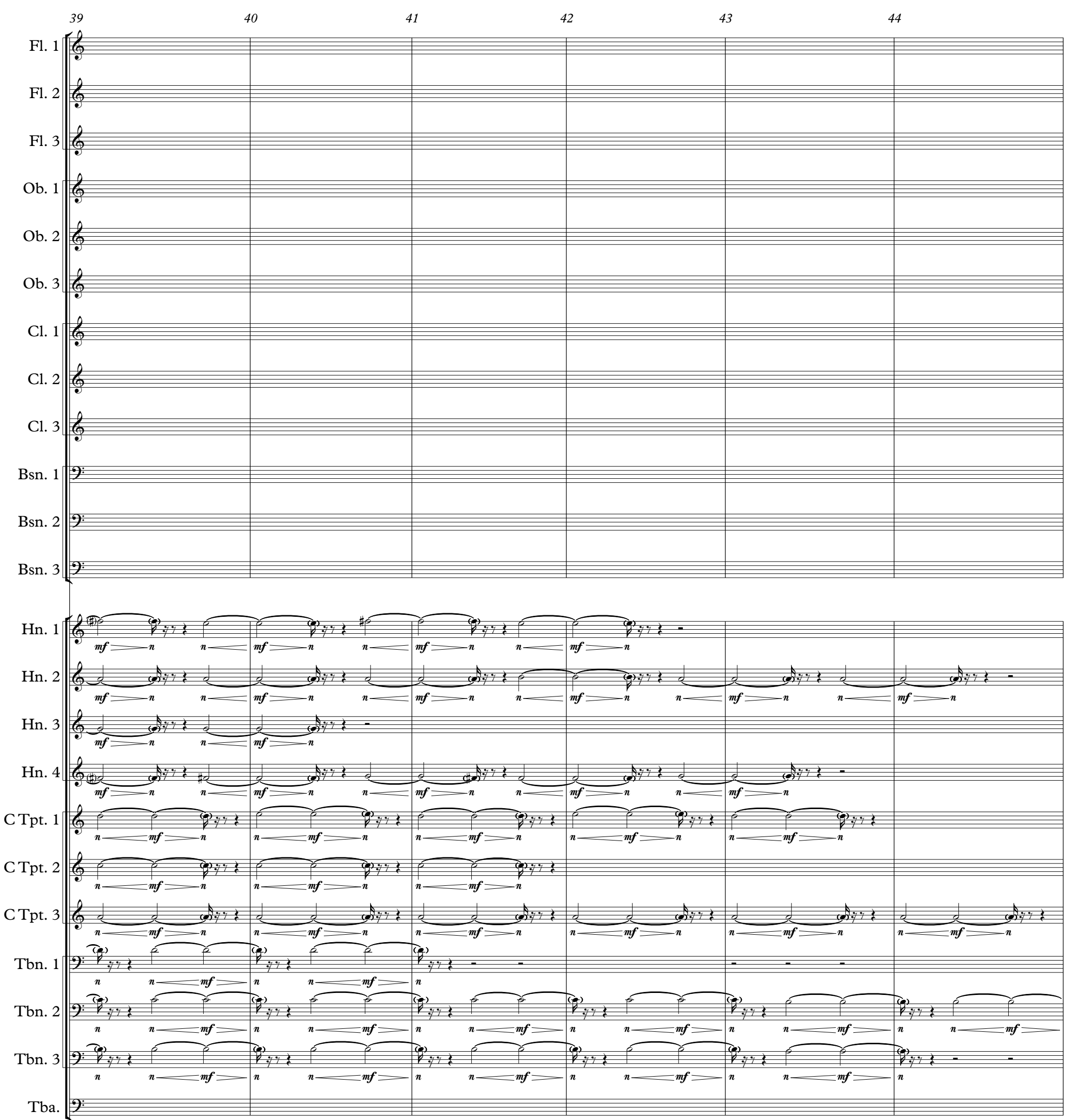

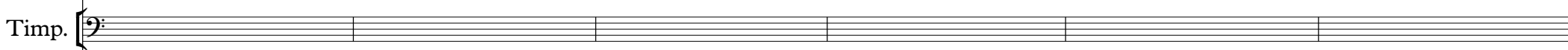

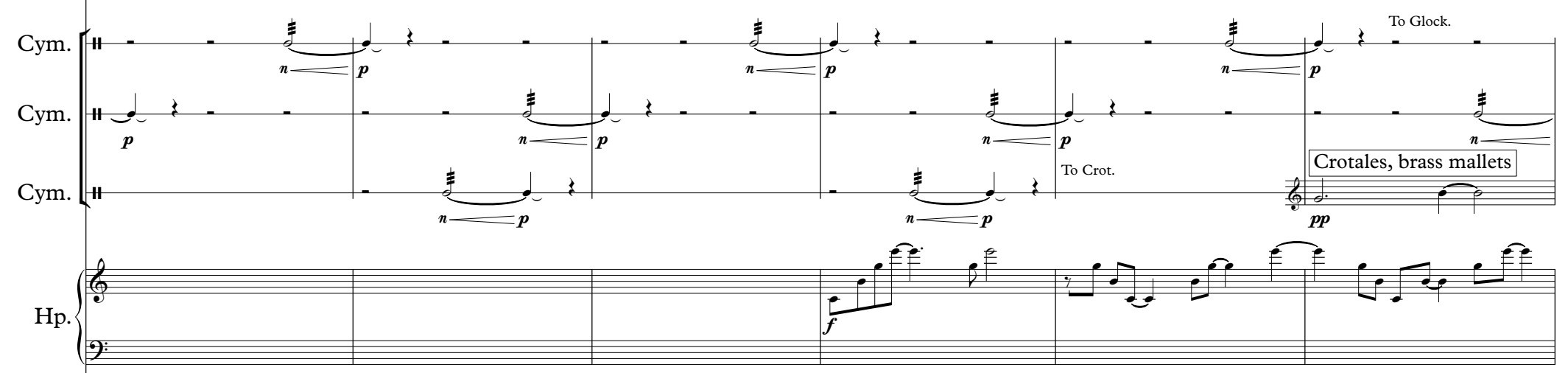

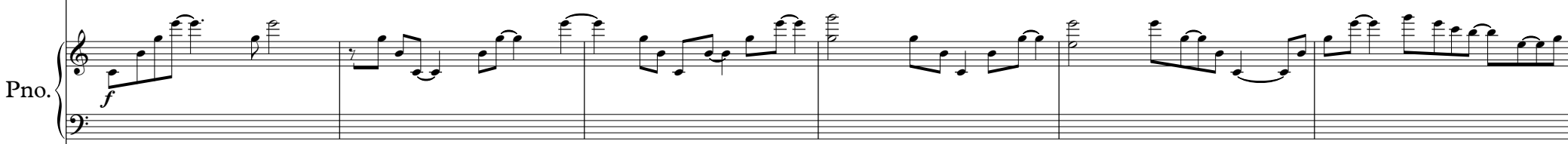

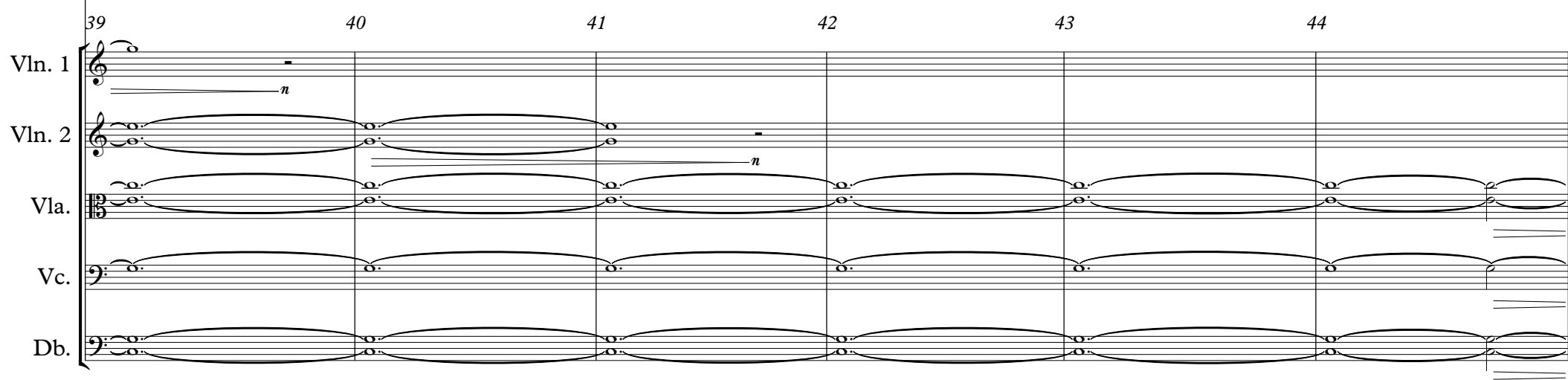



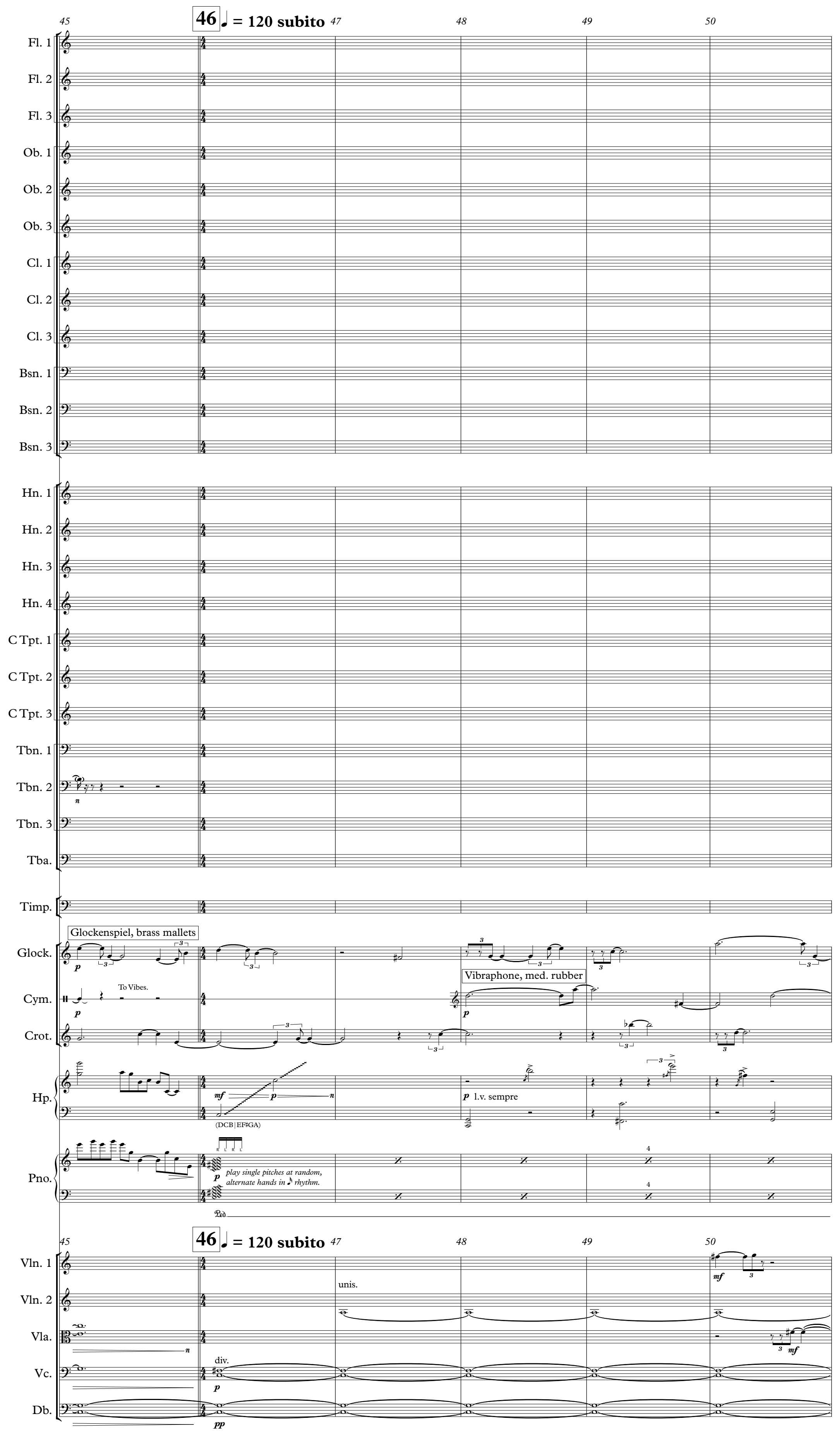

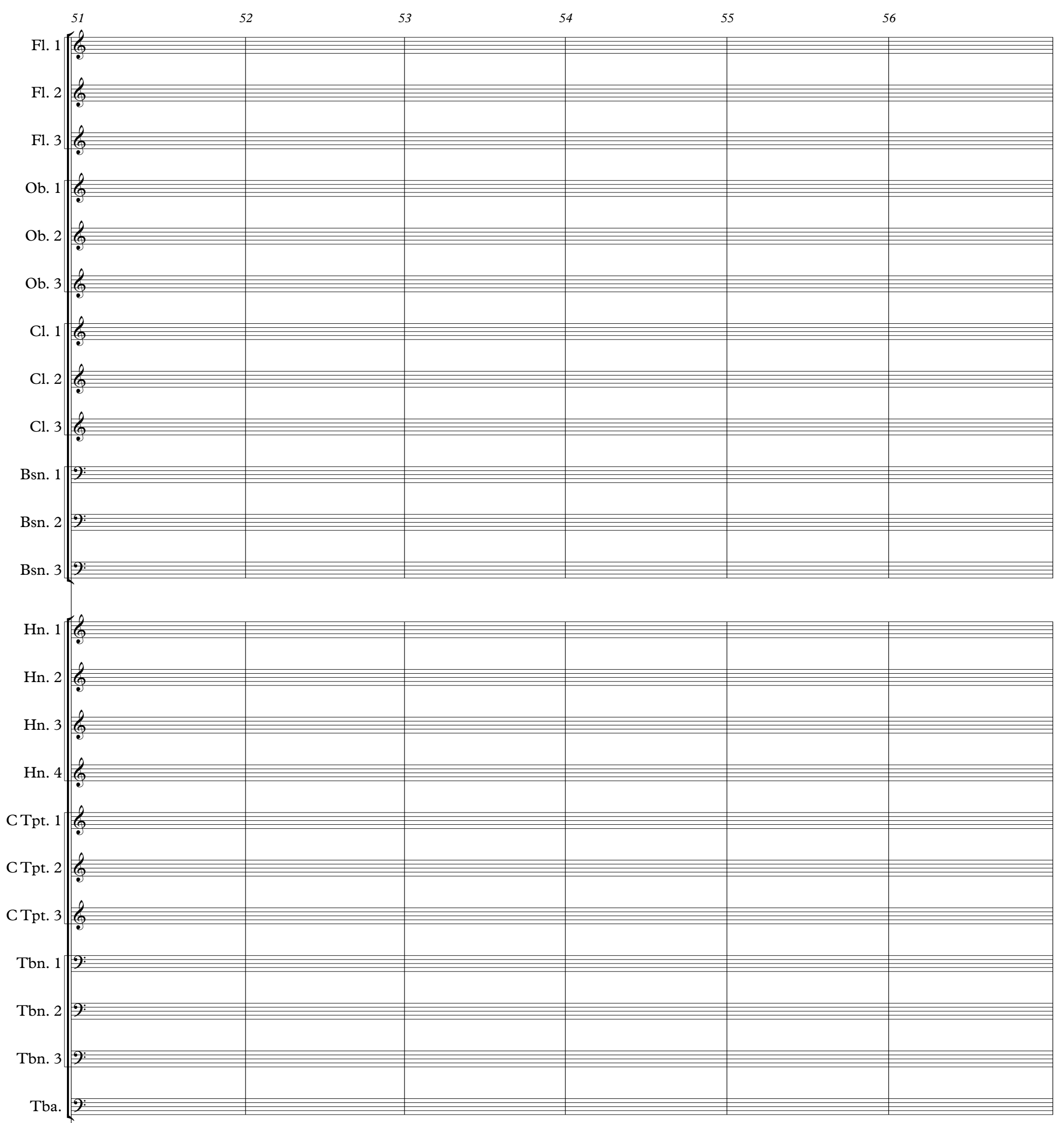

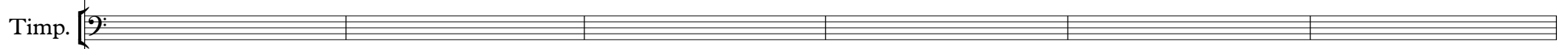

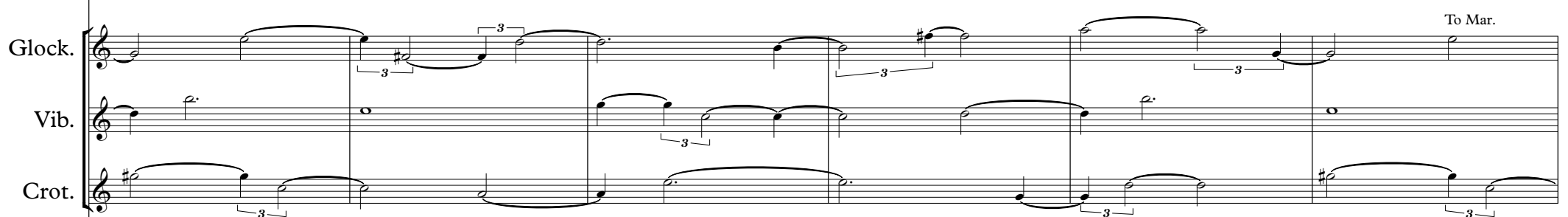

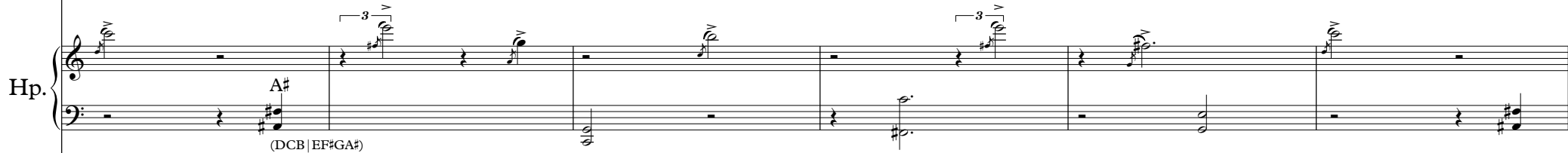

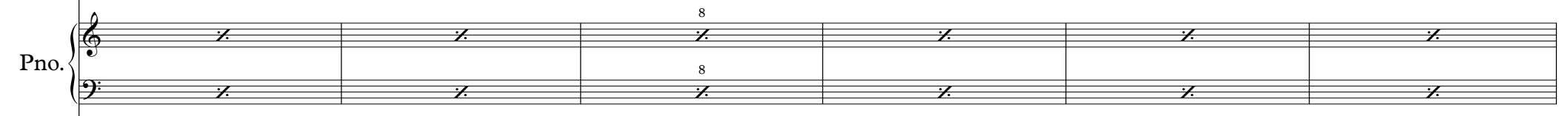

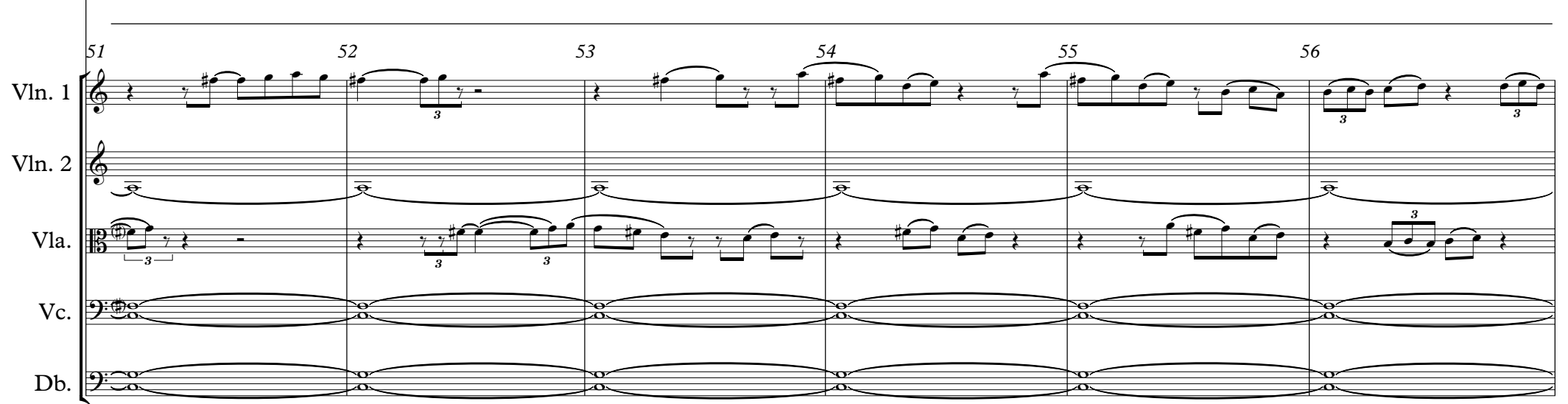



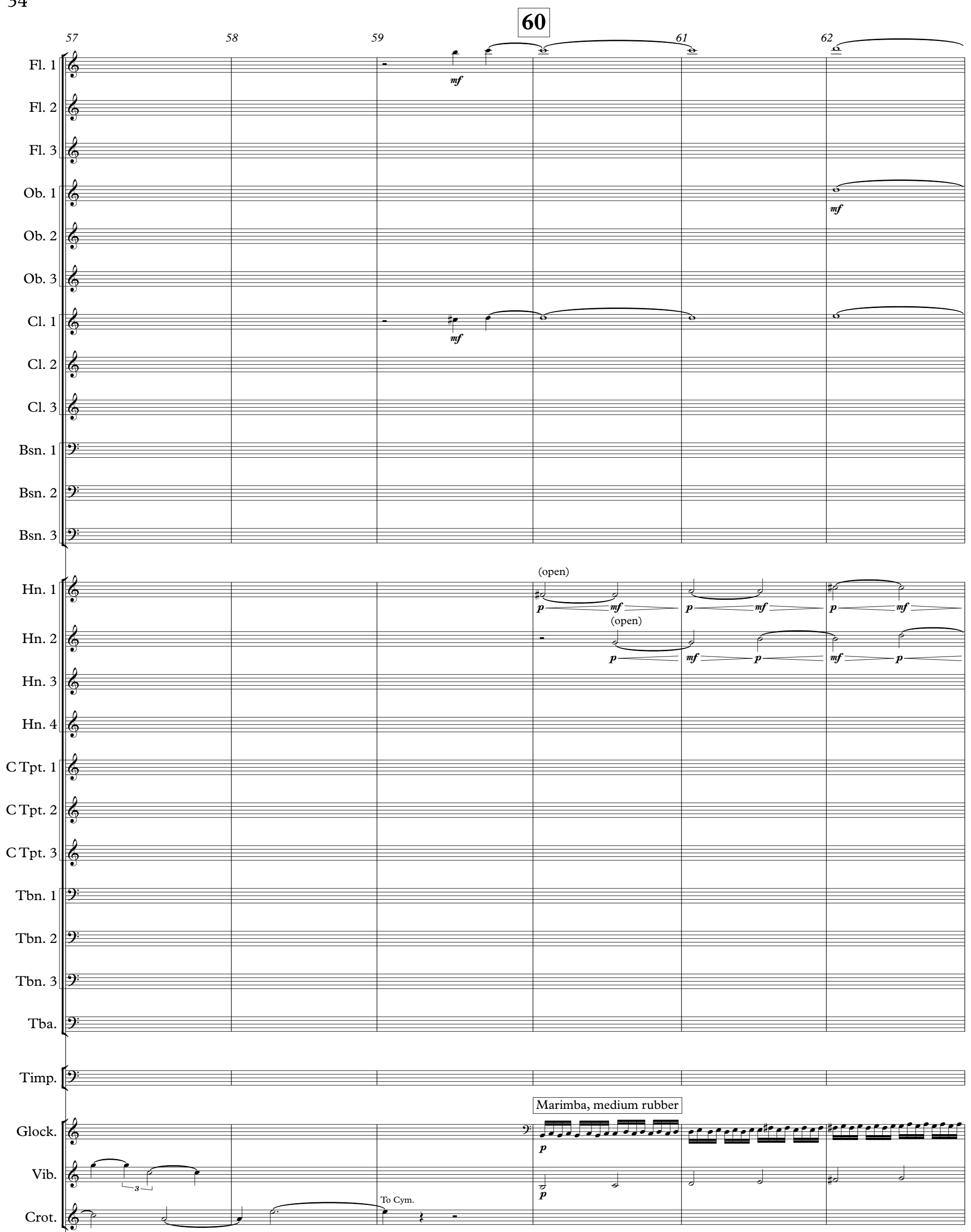

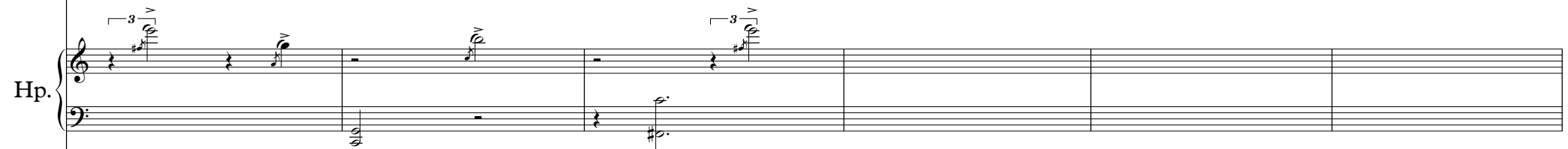

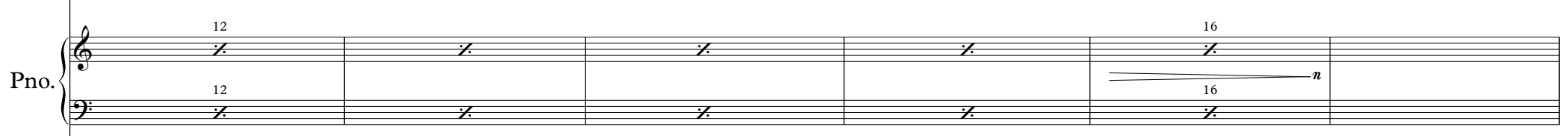

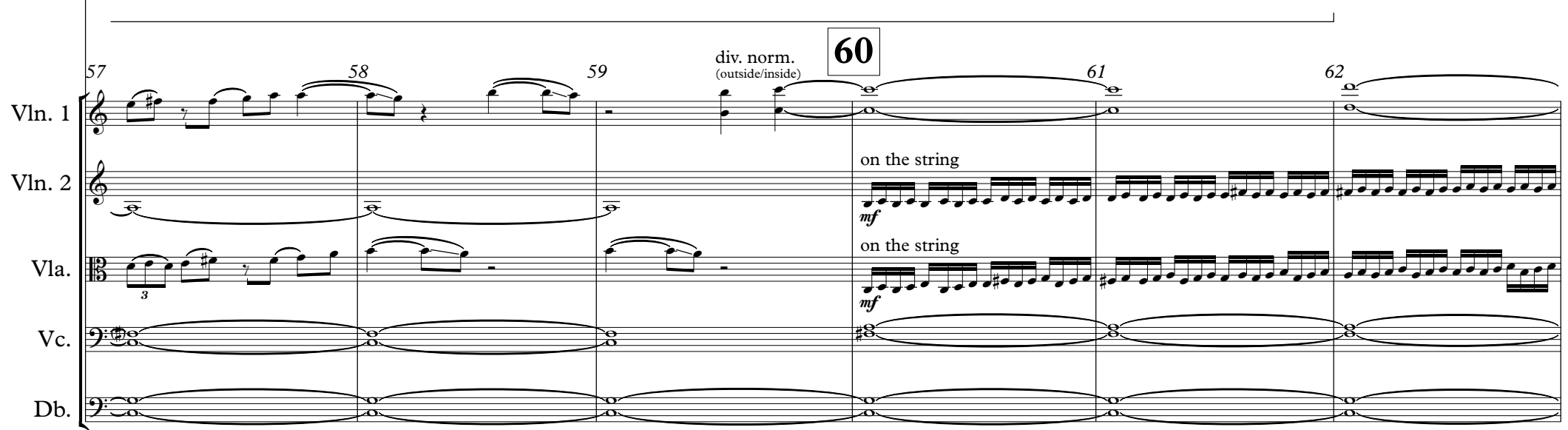



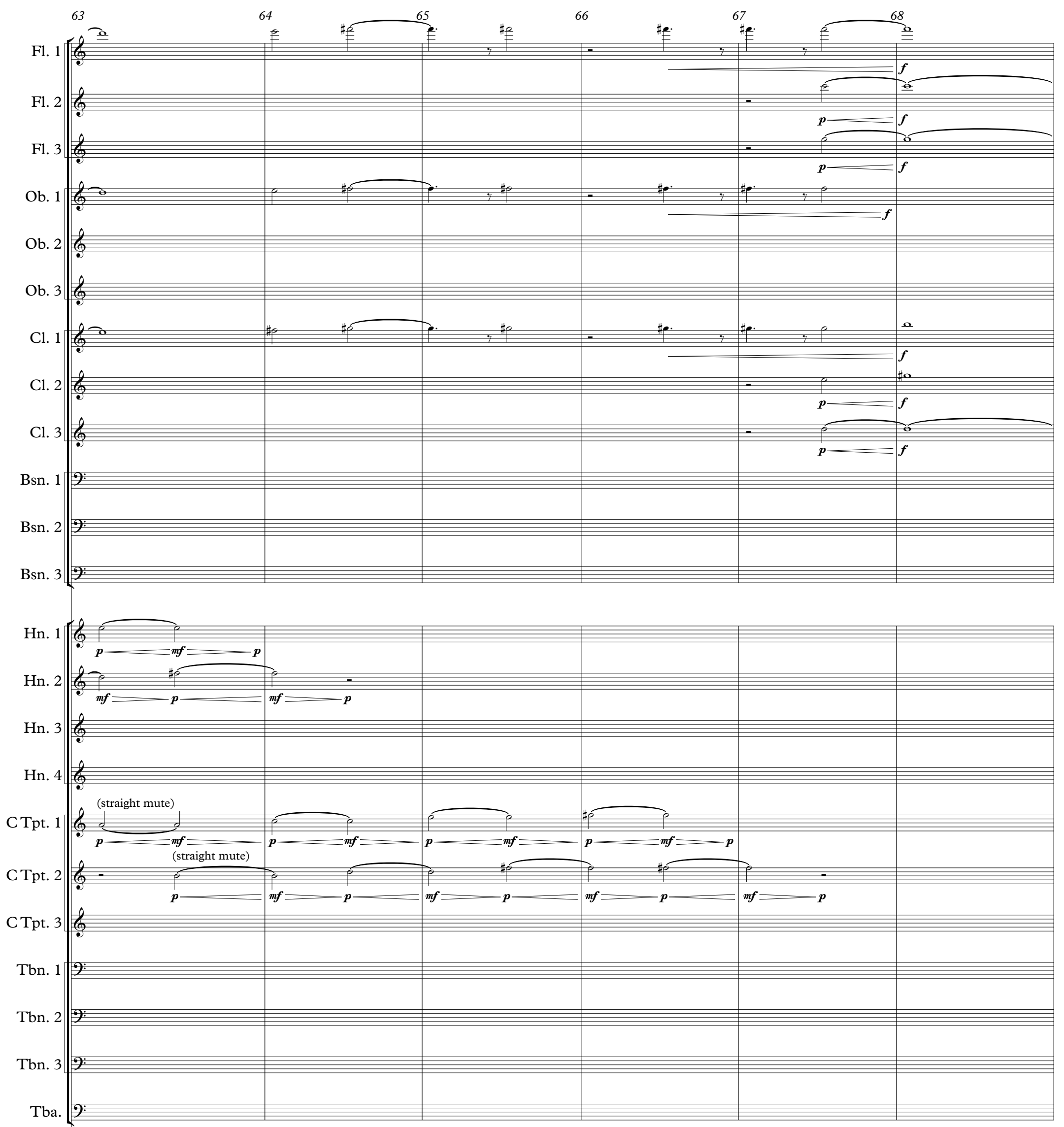
Timp. 2
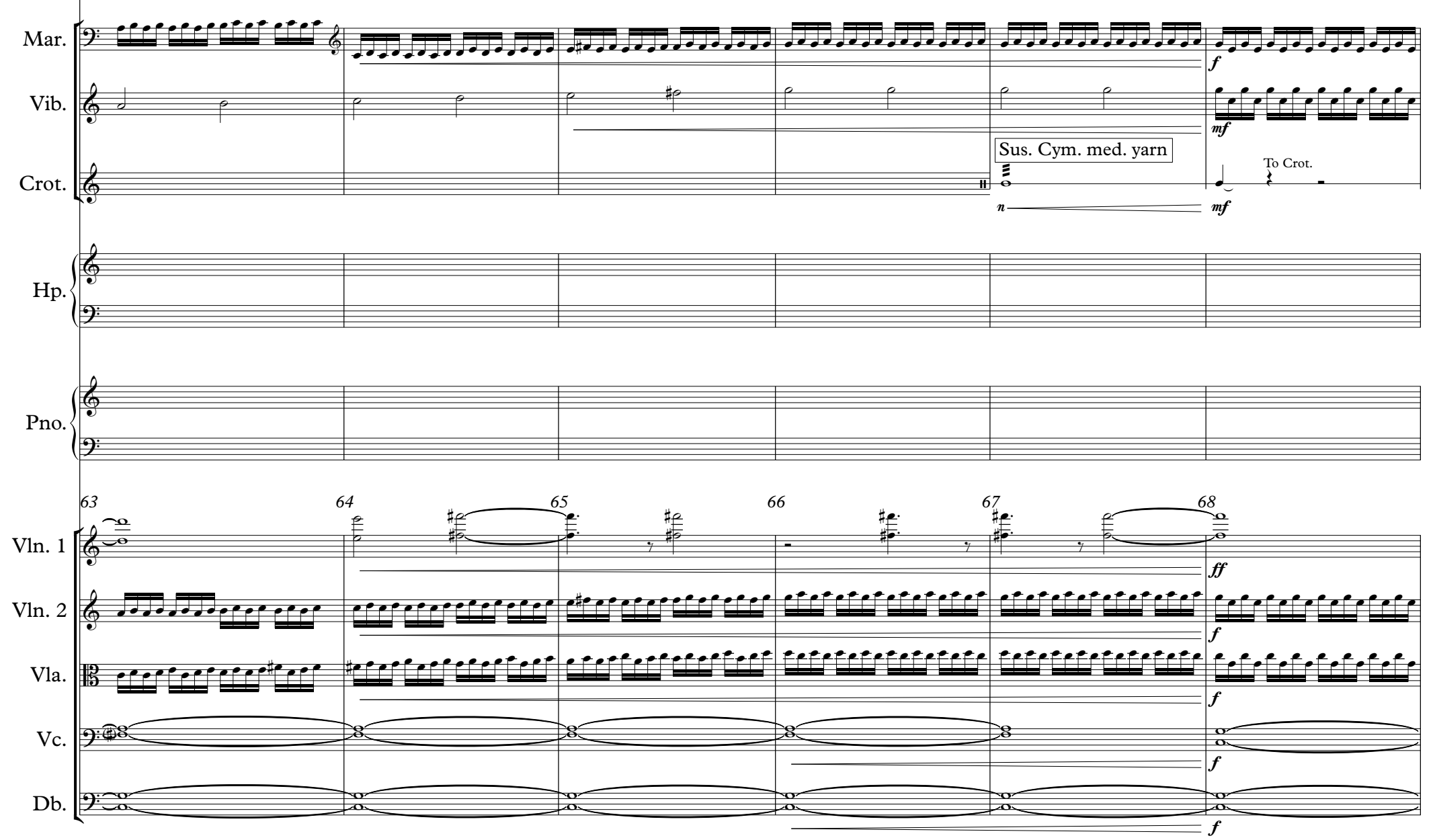

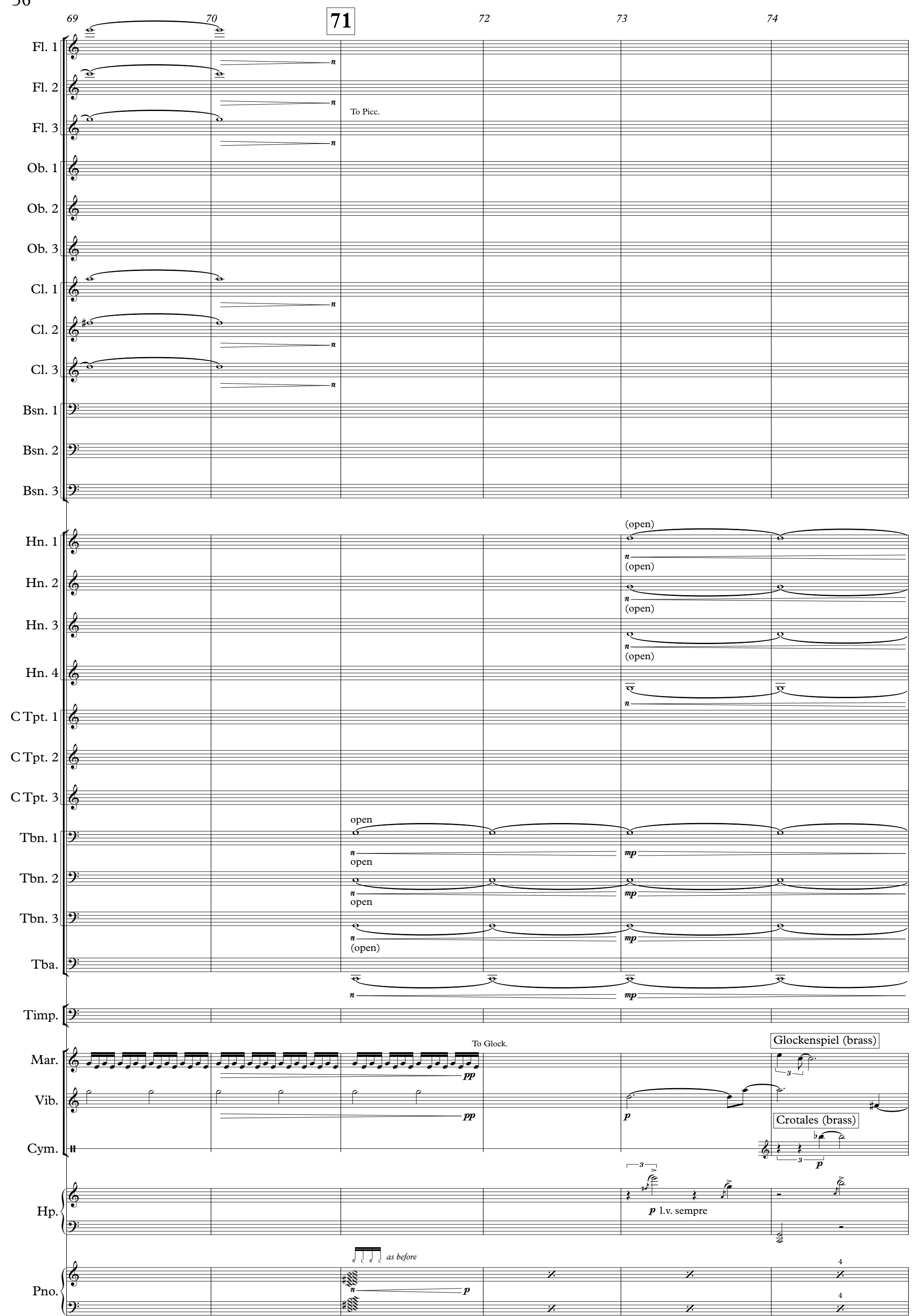

$\sqrt{I ! n !}$ as before

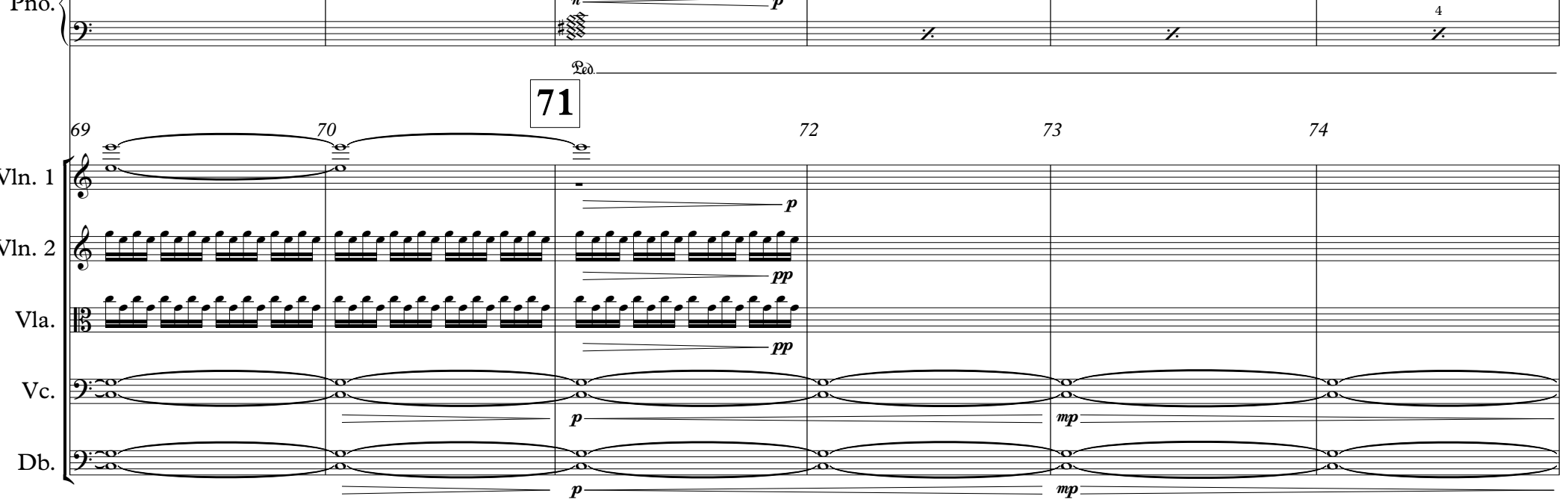



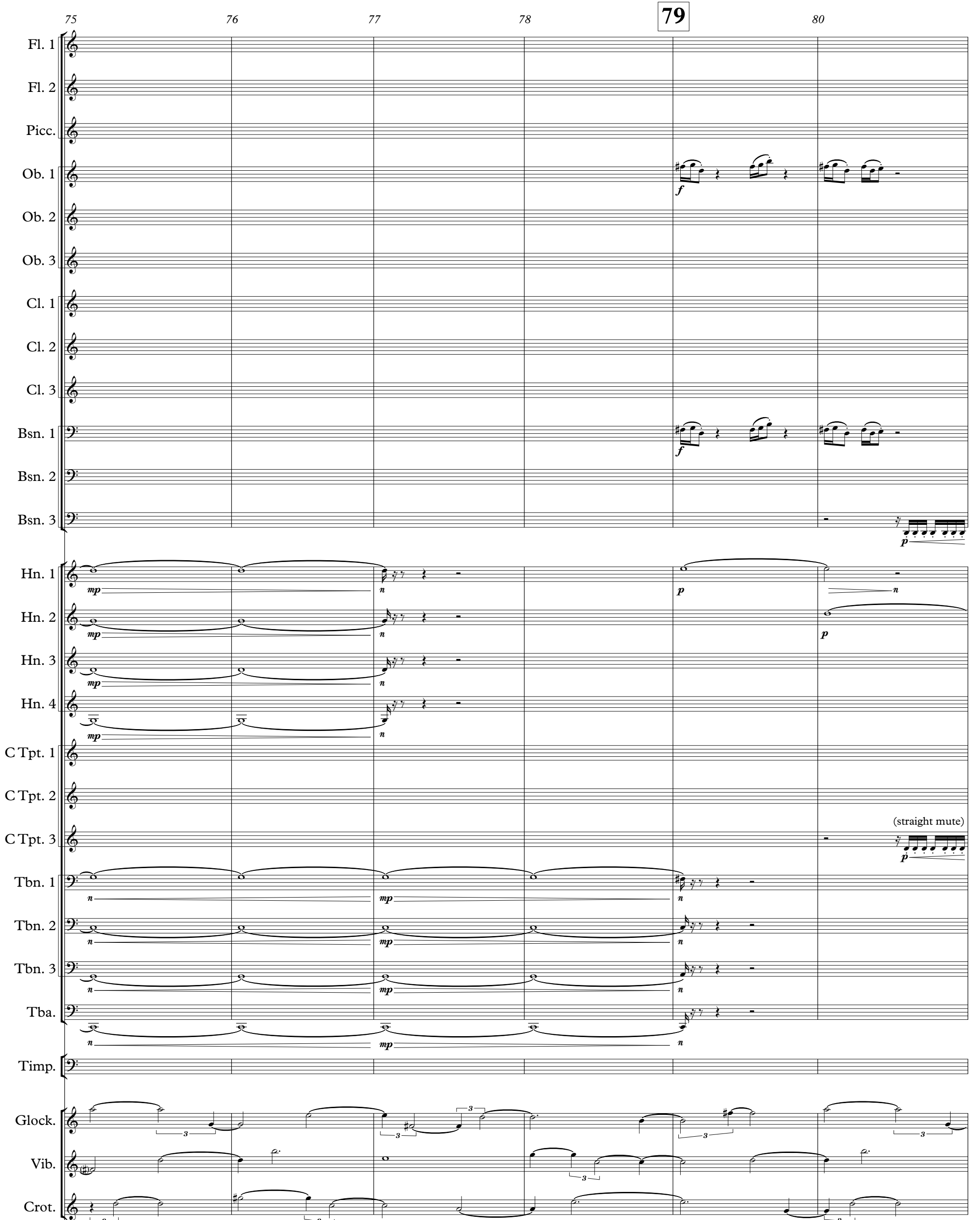

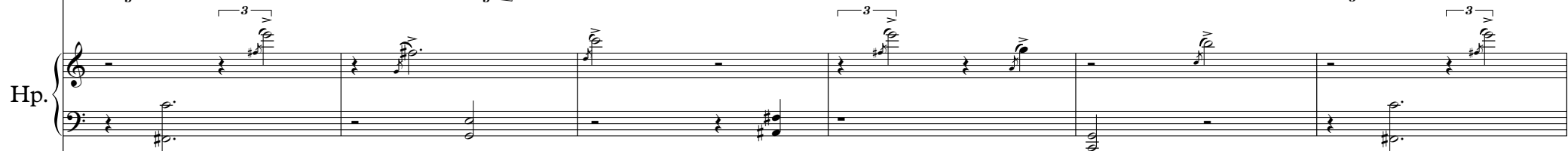

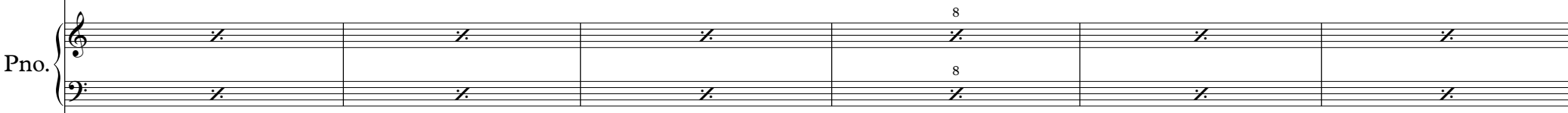

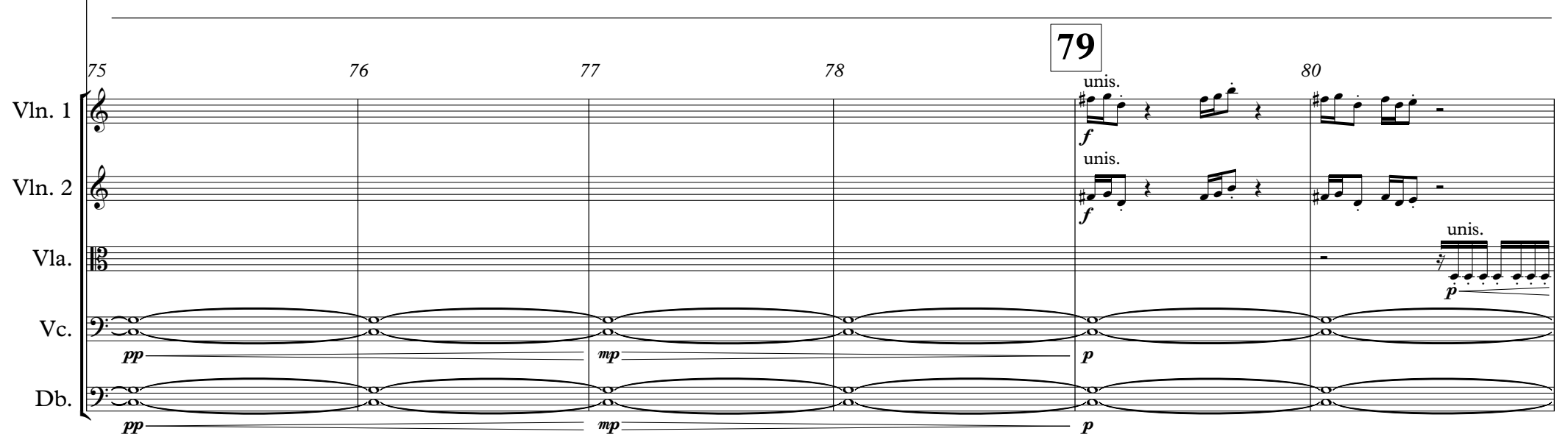



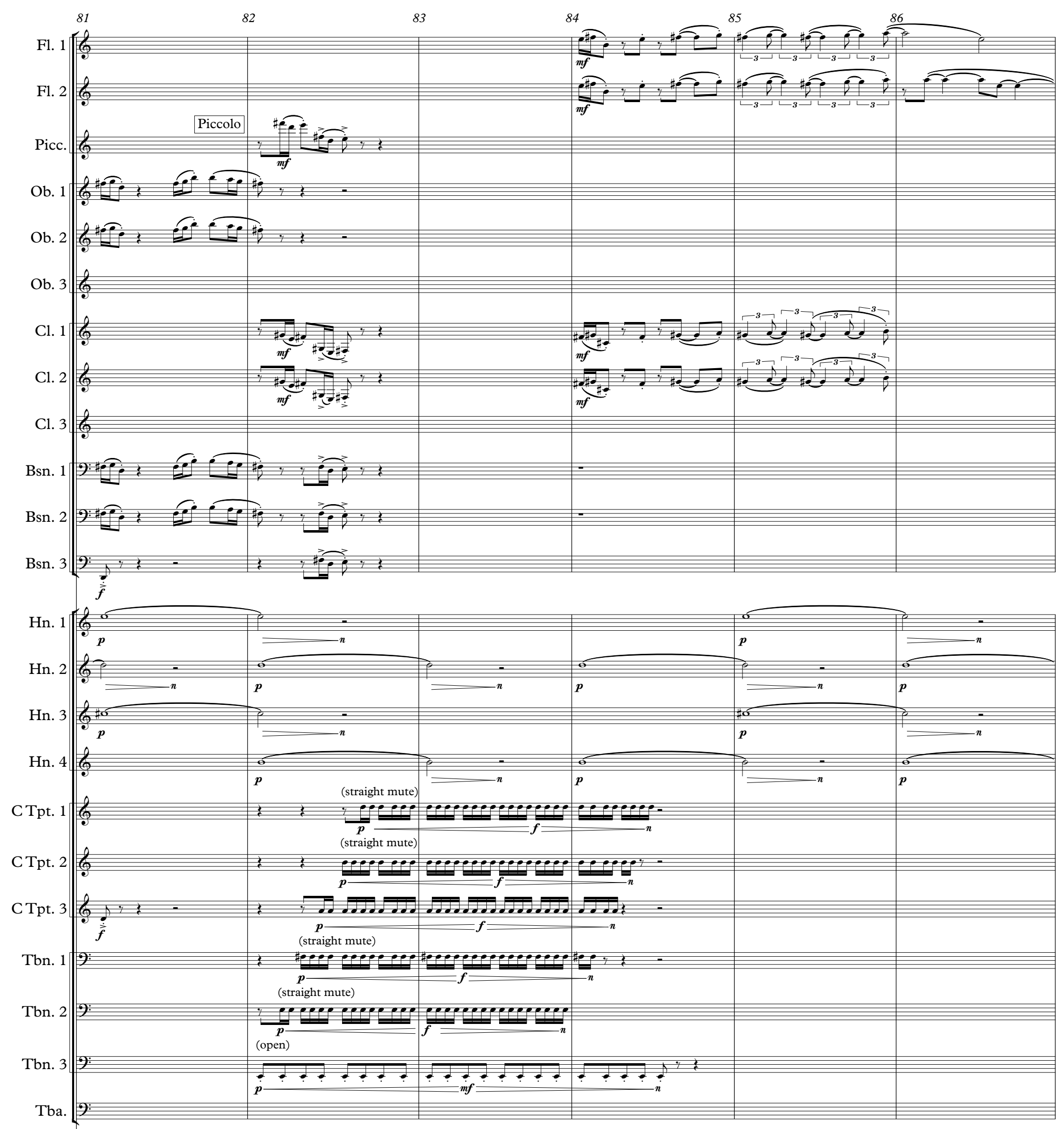

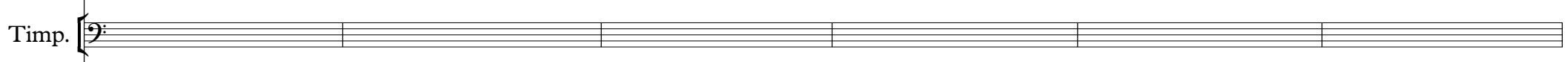

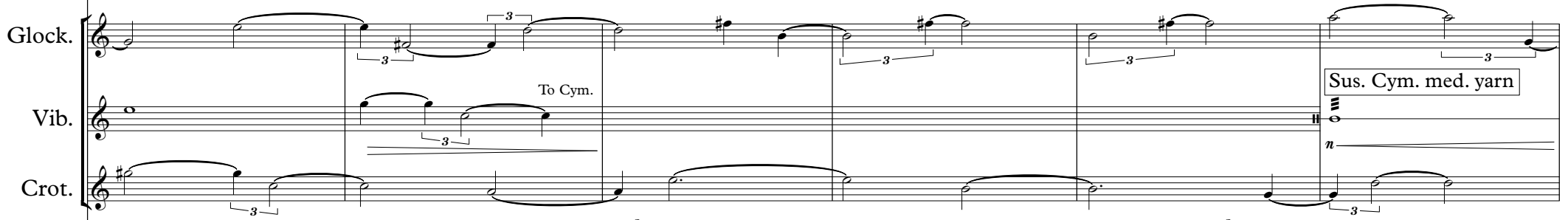

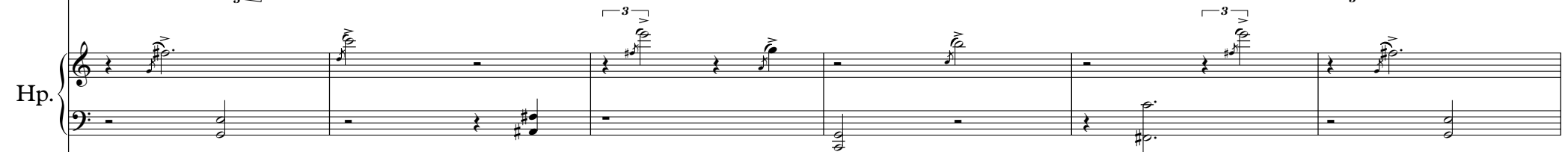

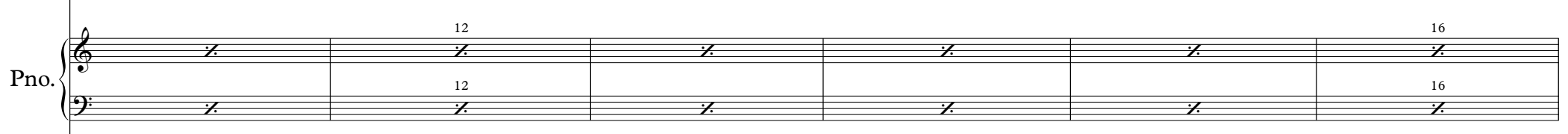
VIn. 2 DIn 1 

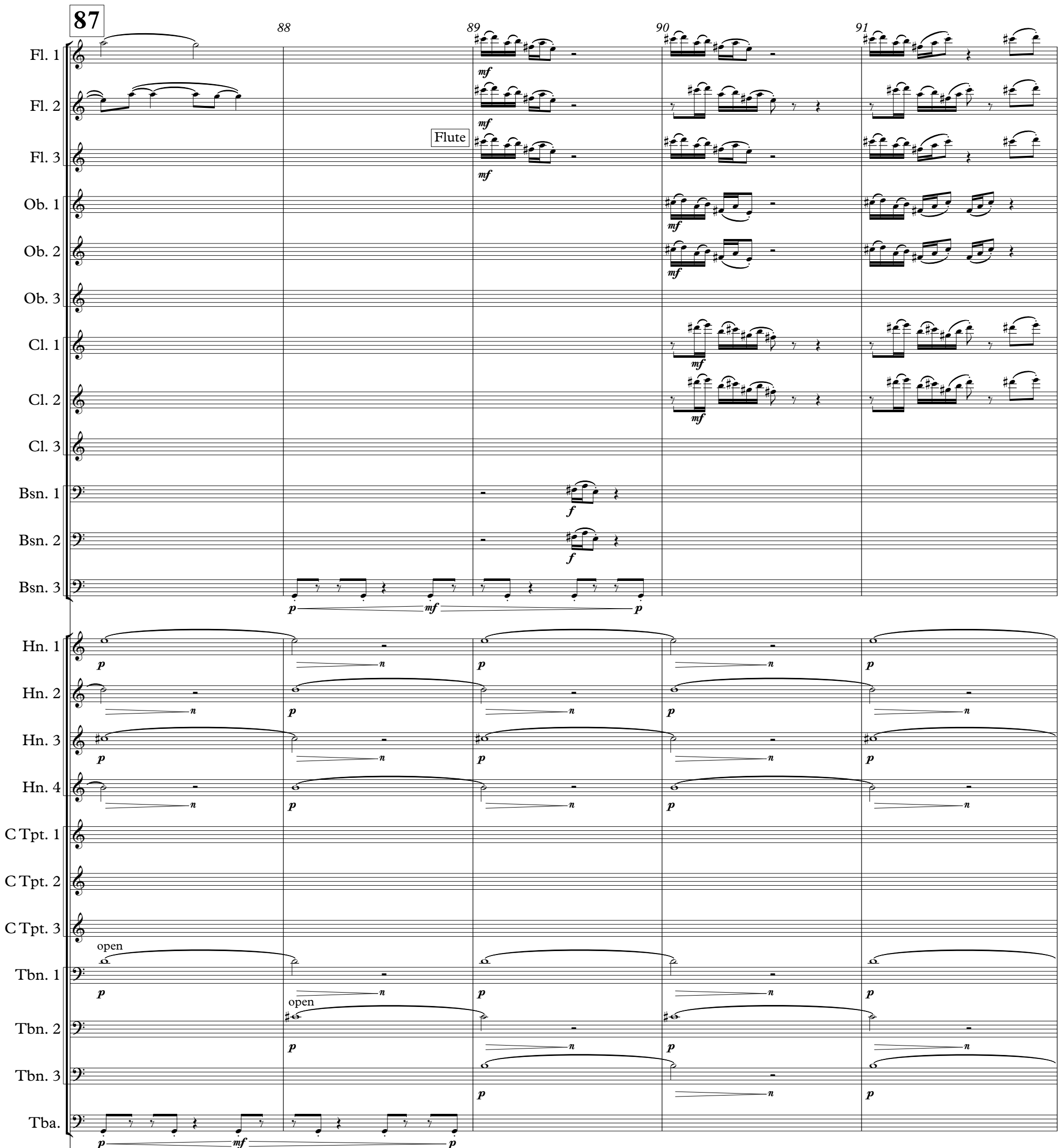

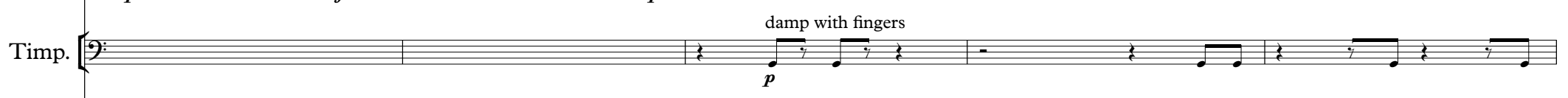

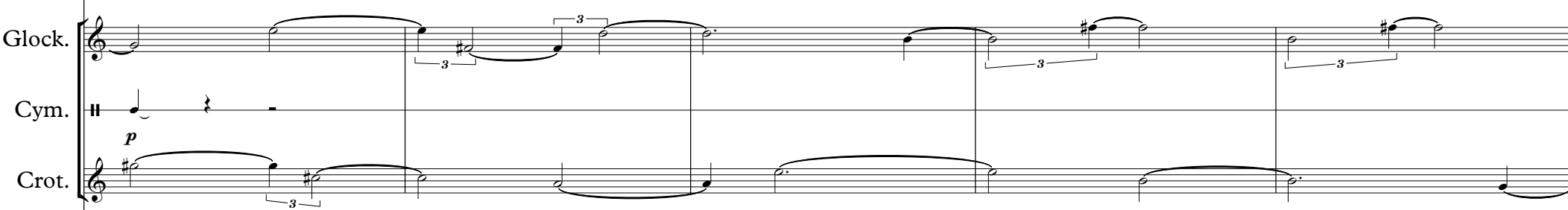

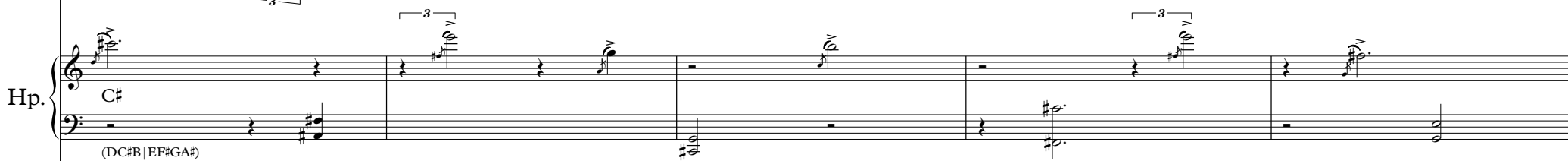

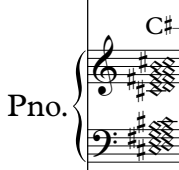

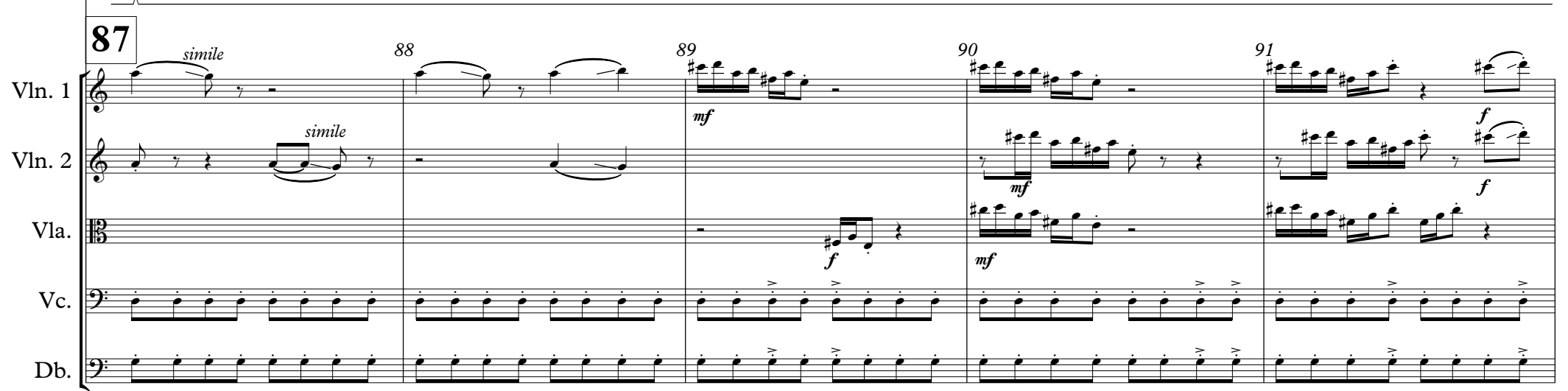


40
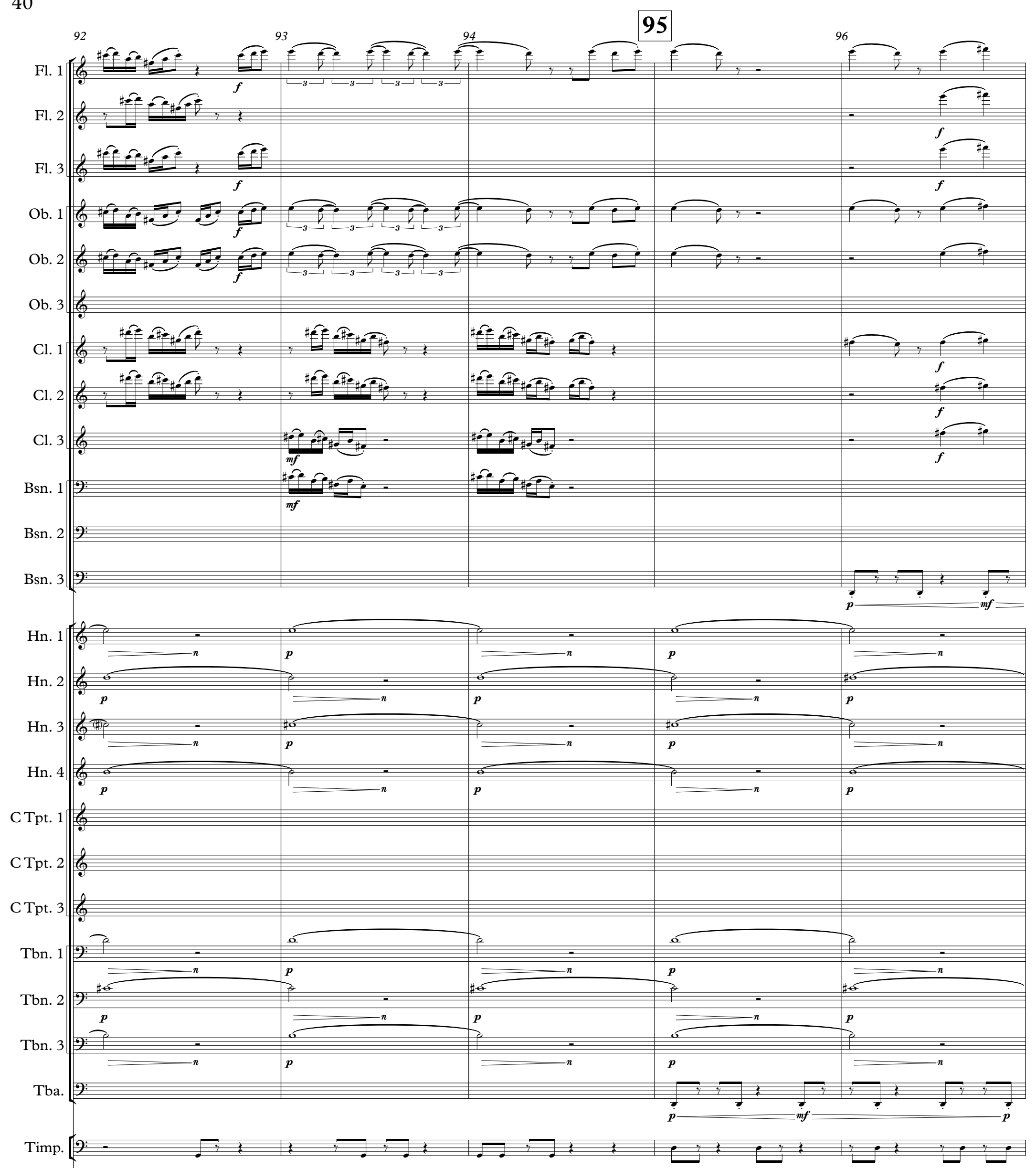

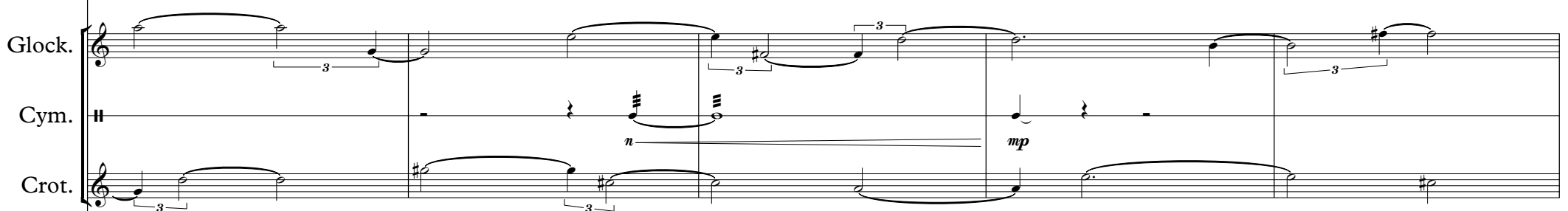

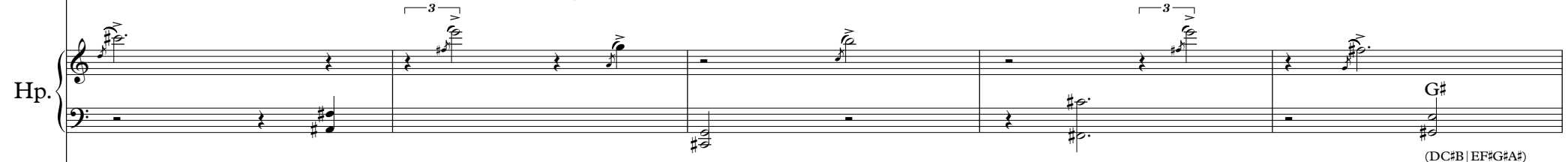
B 

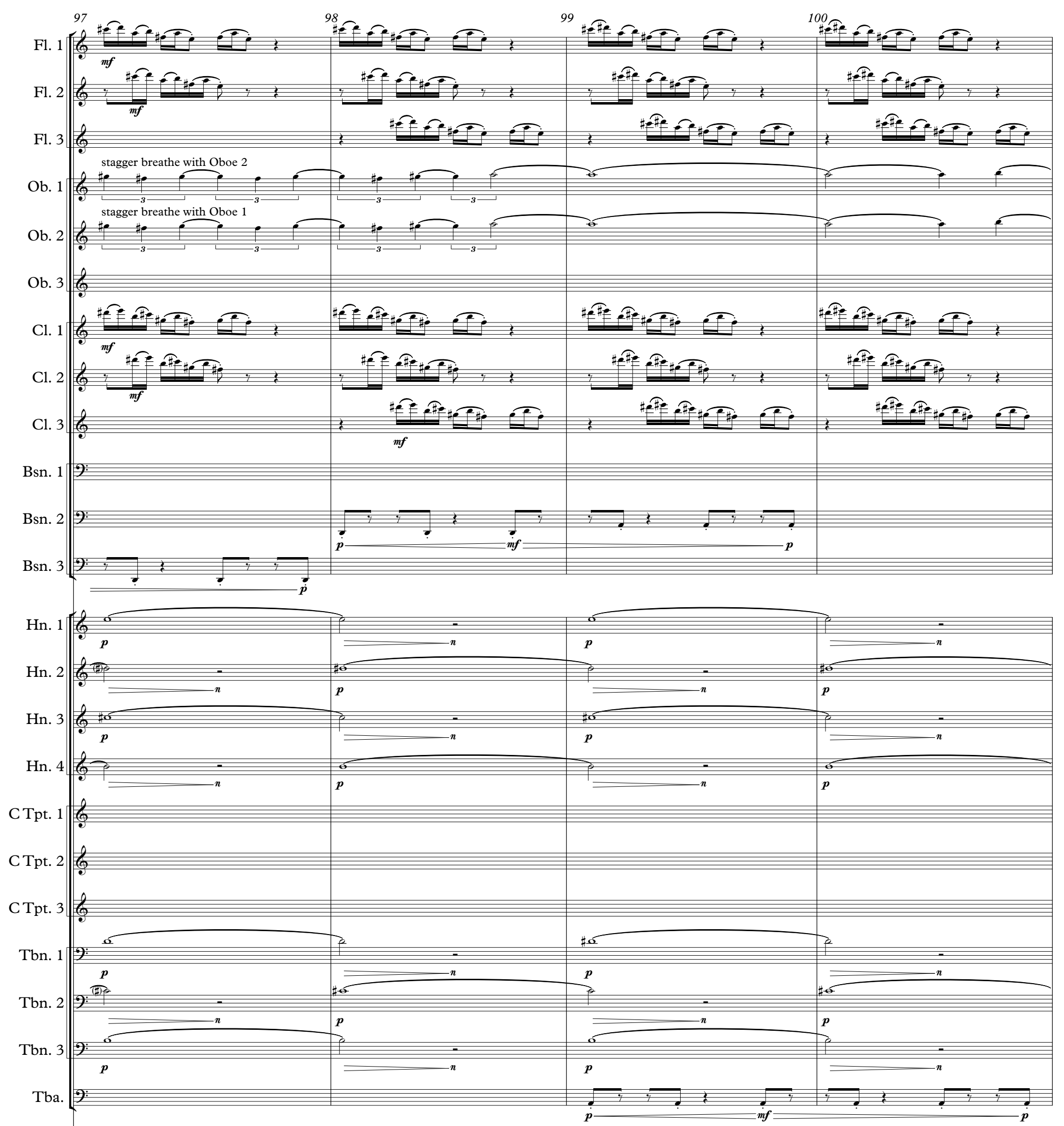

Timp. Cym.

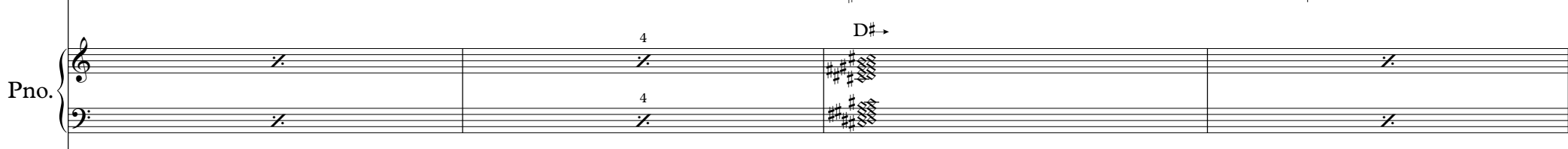
VIn. 2 VI. 


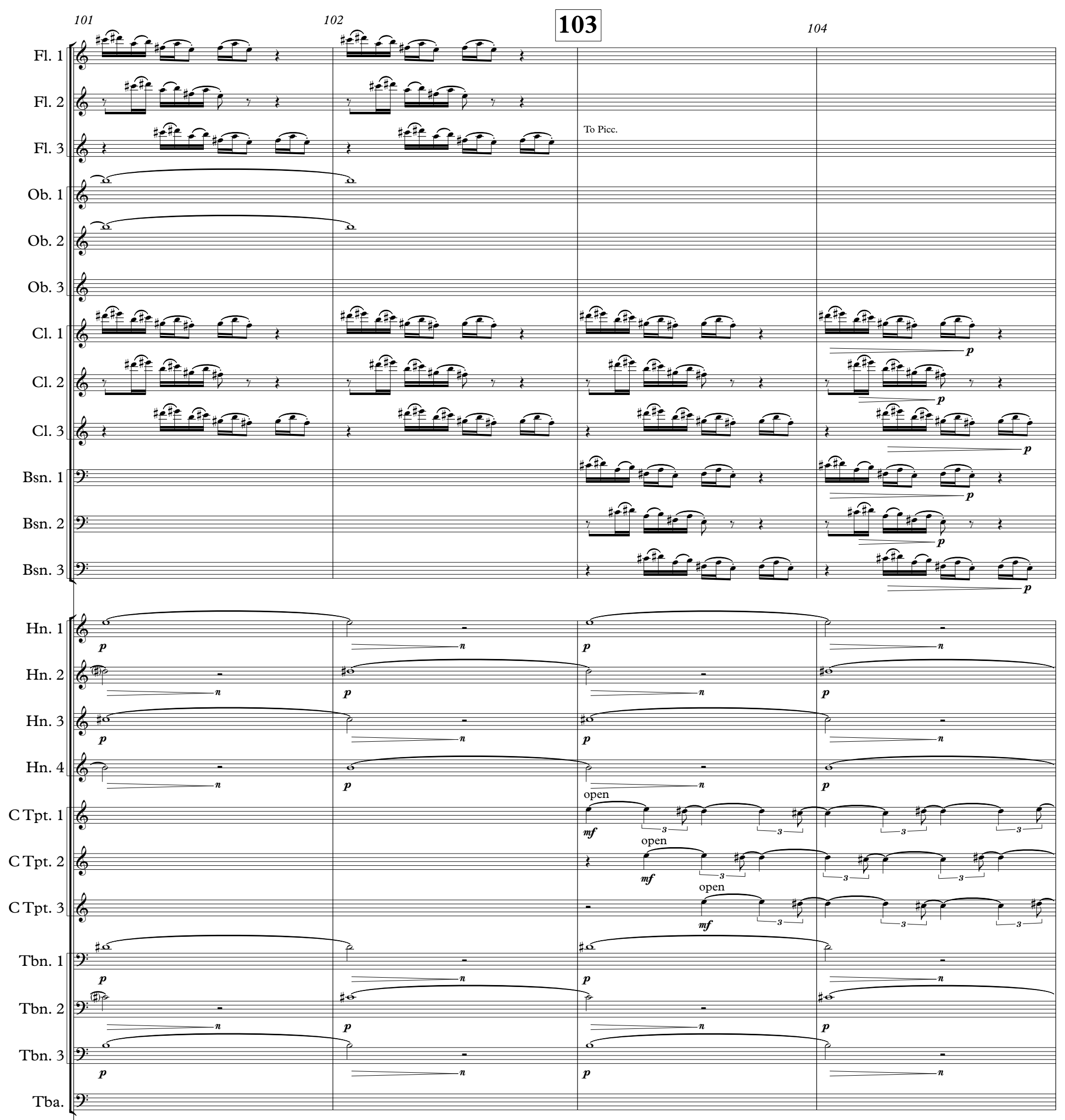

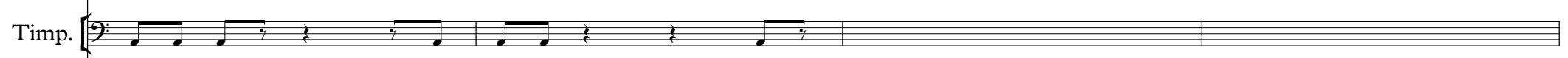
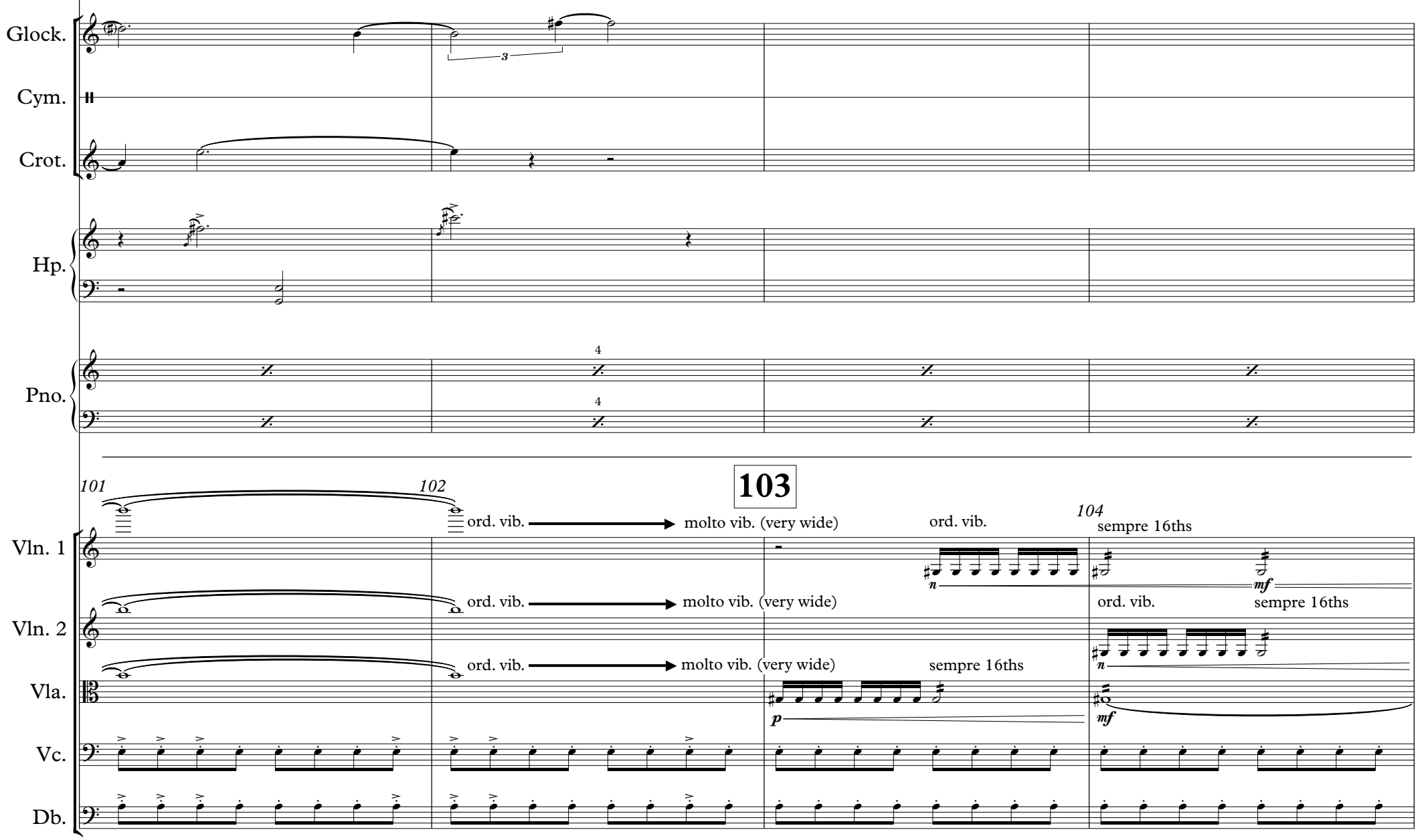

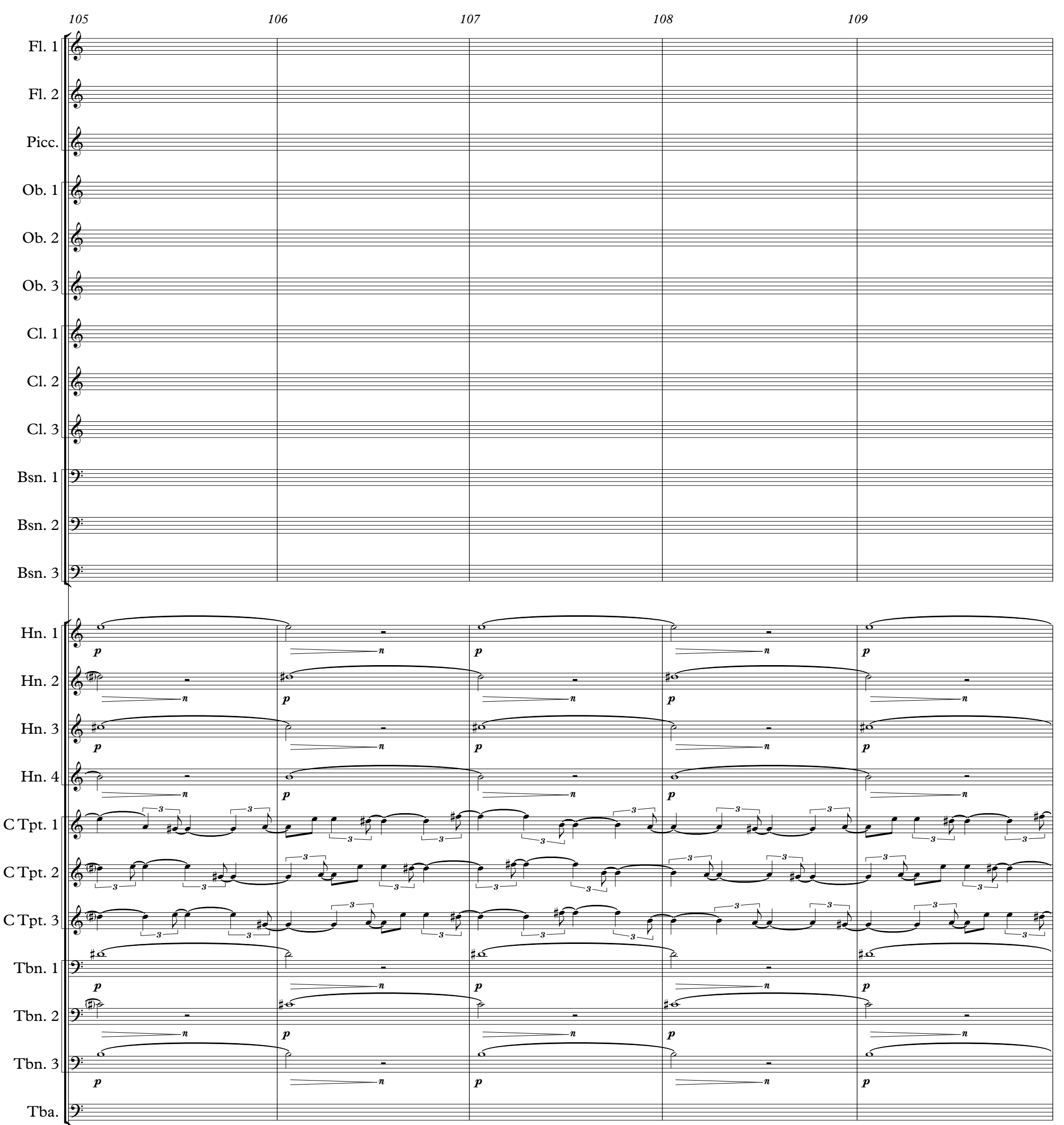

Timp.

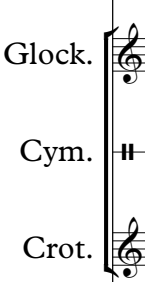

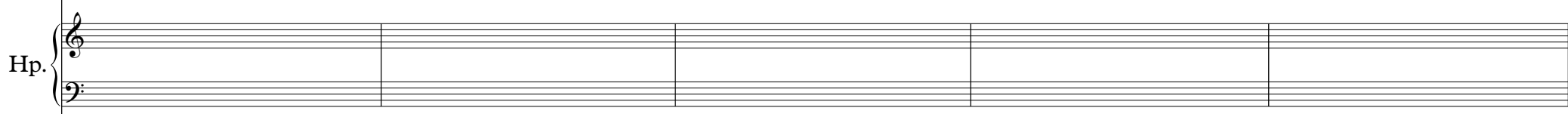

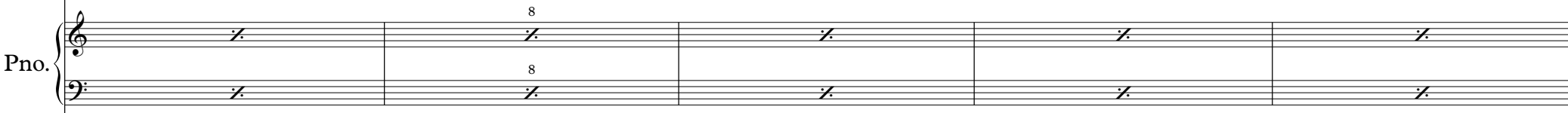

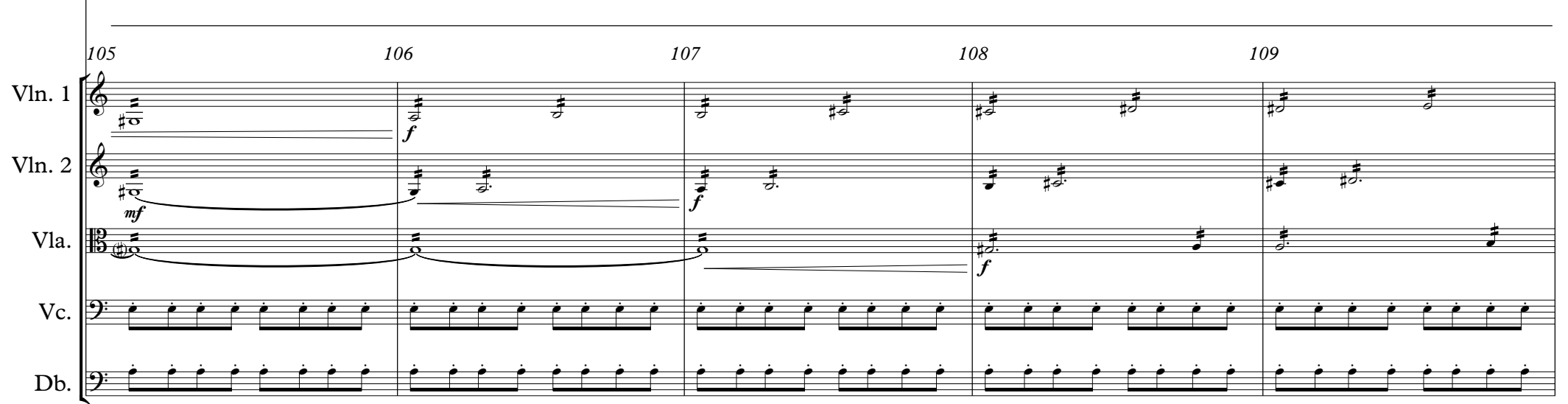



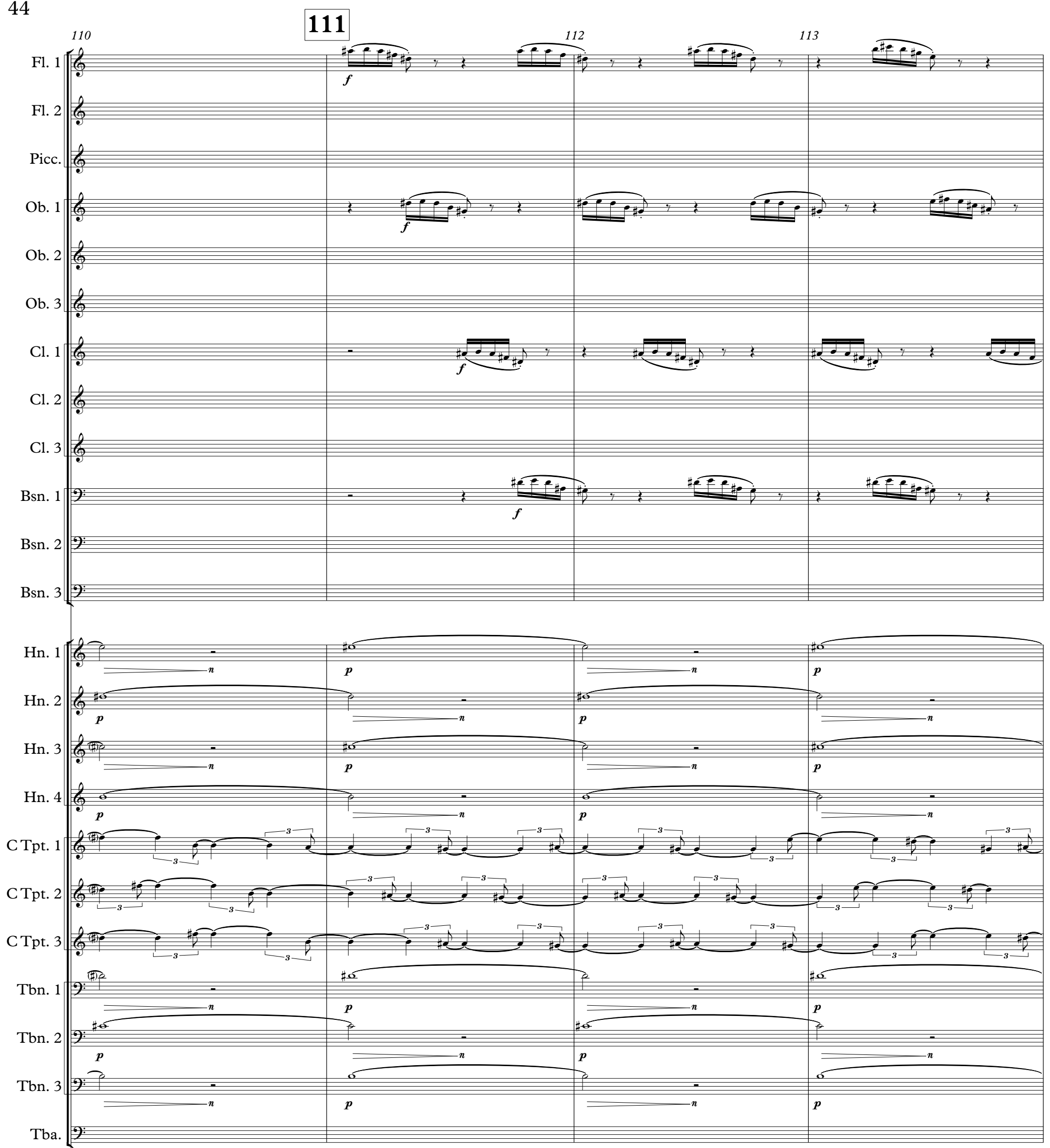
Timp.
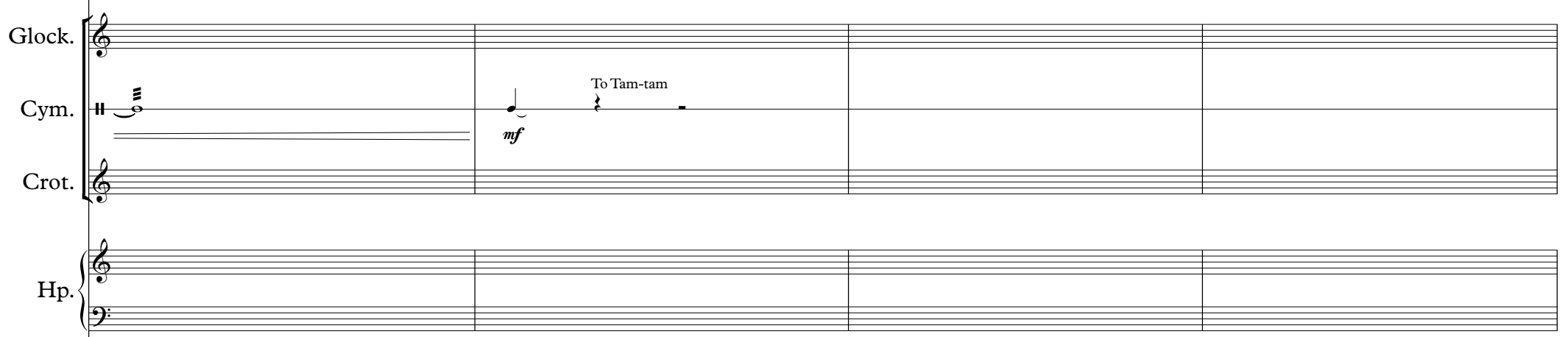

Pno. $A \#$ -

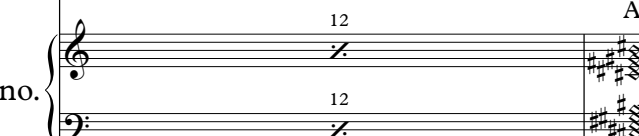

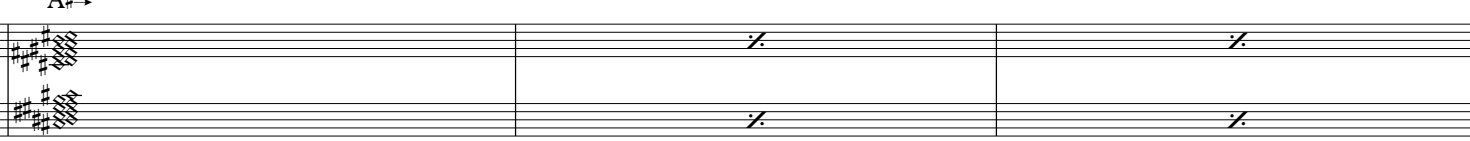

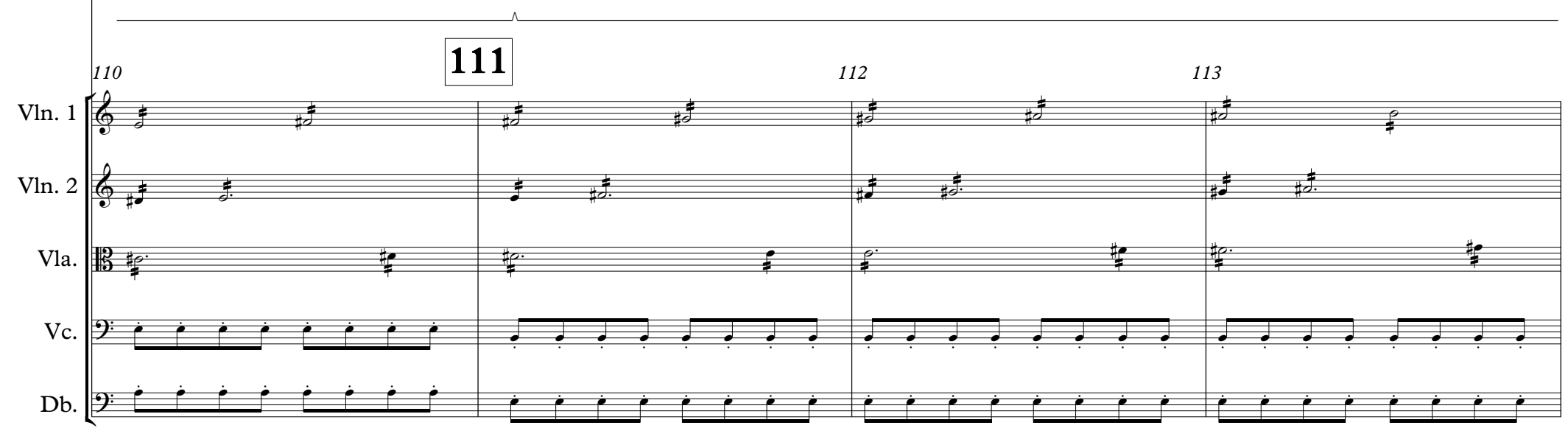



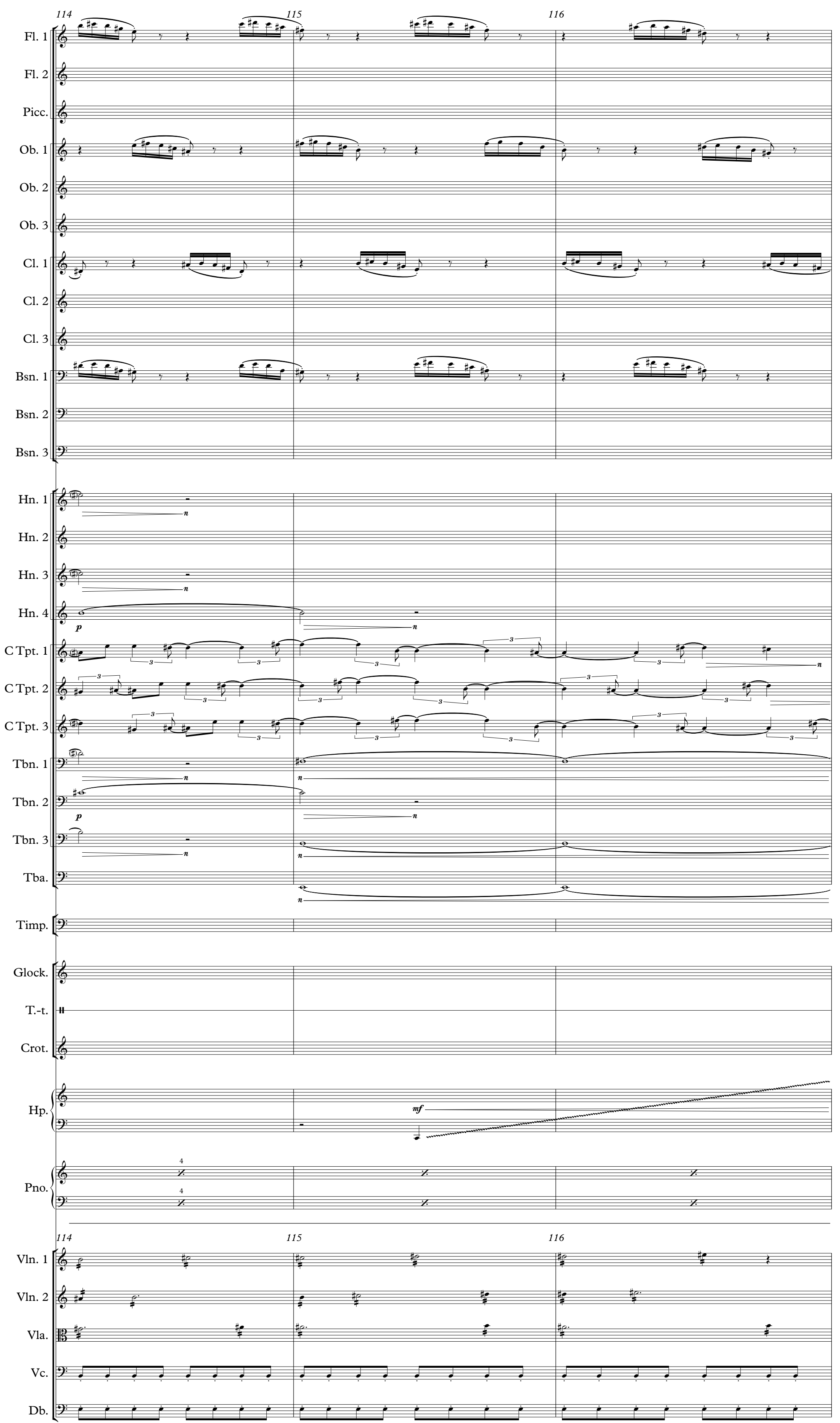

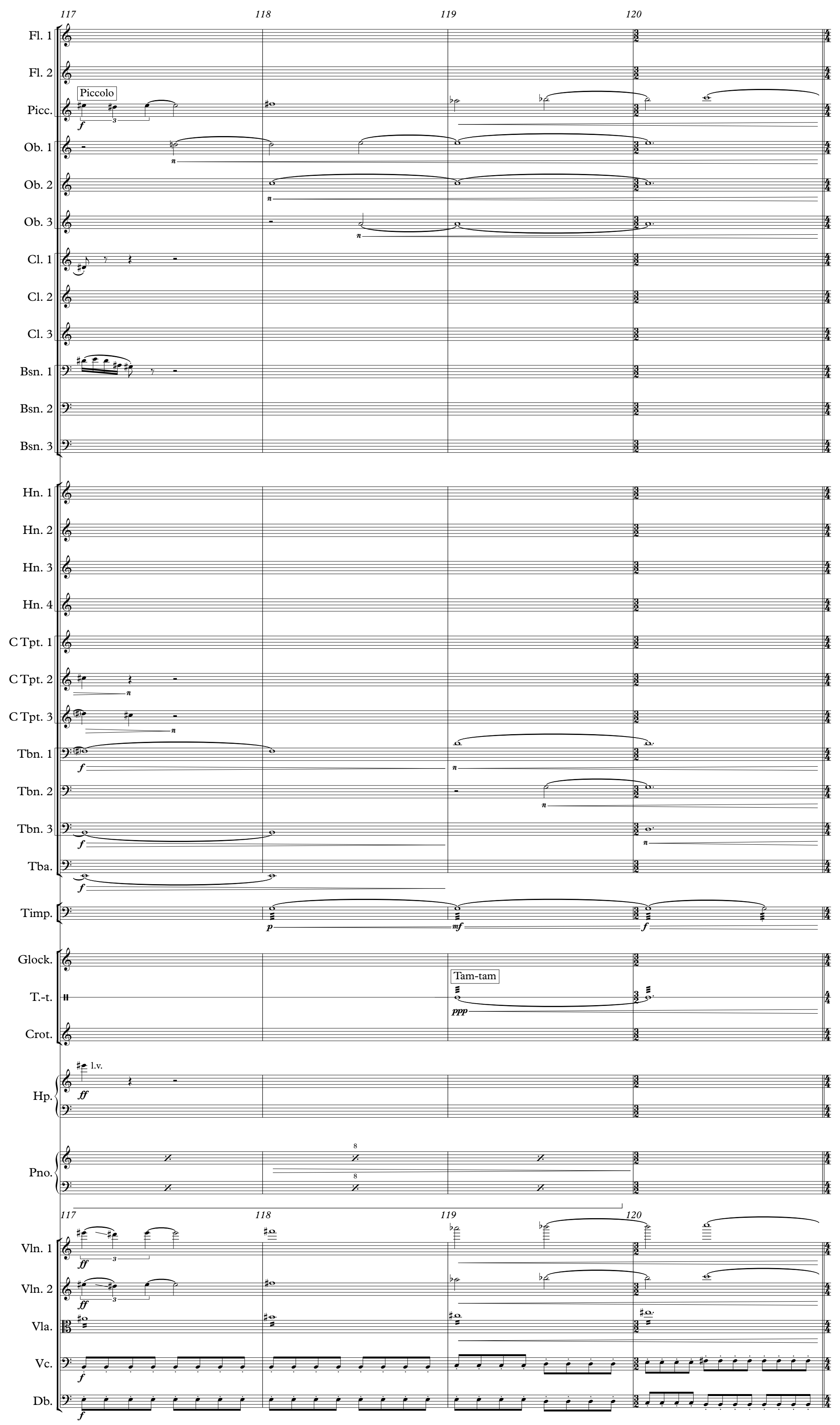

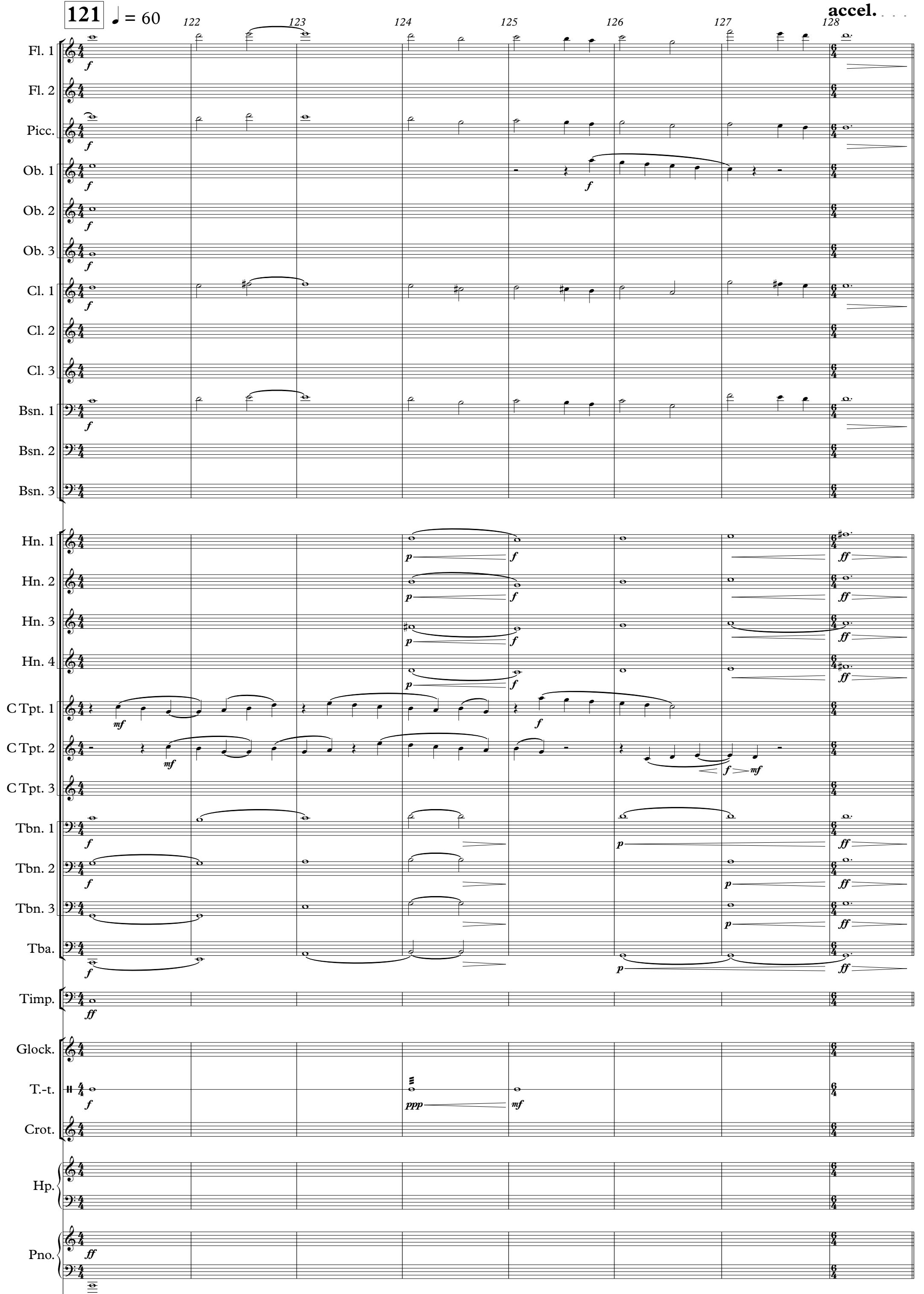

$$
121 \text {. }
$$

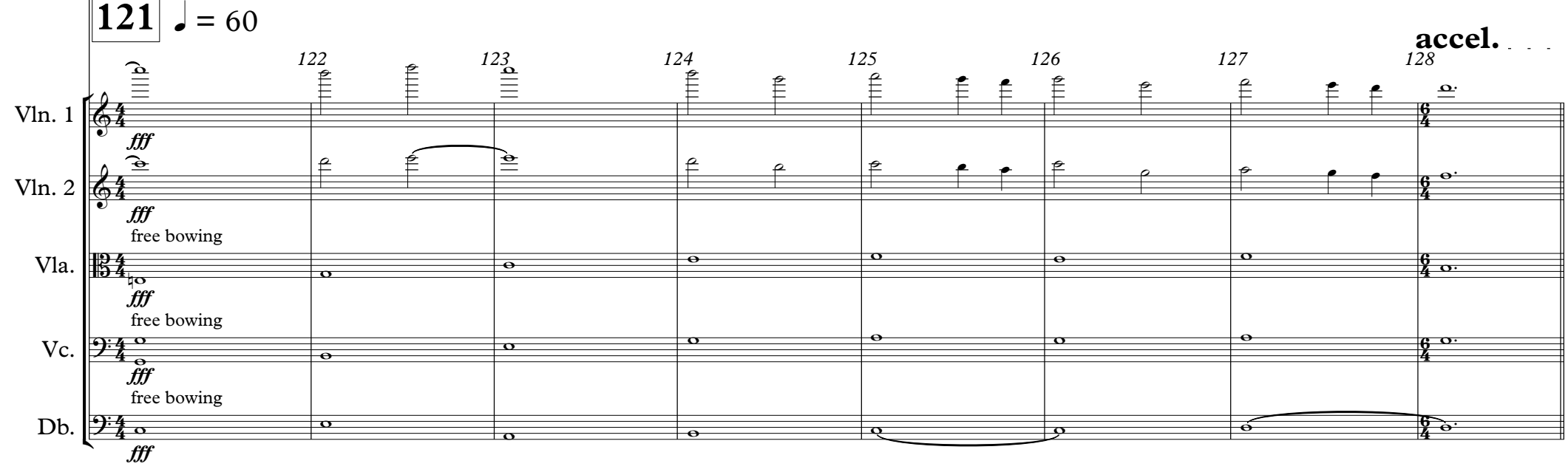



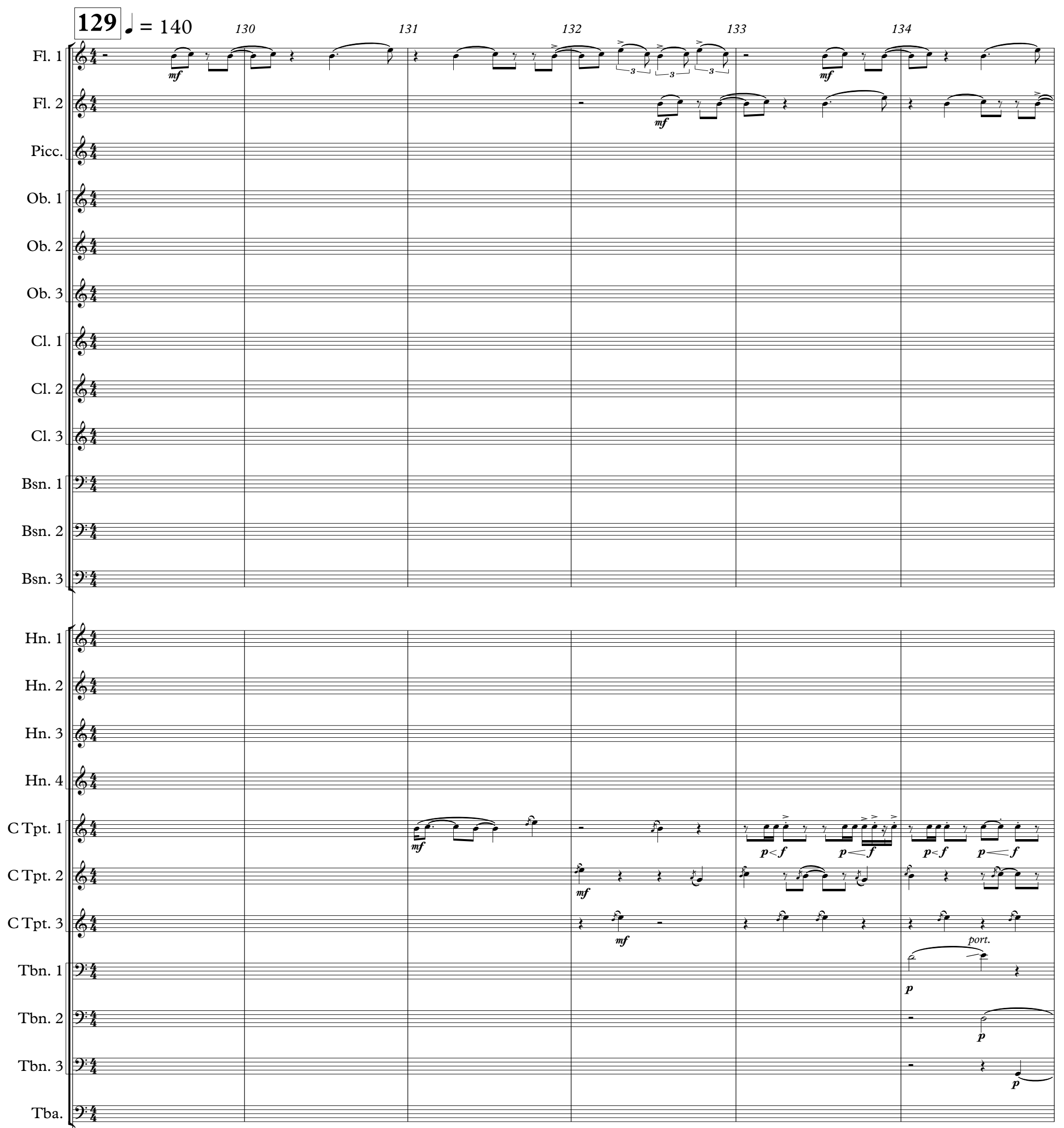

Timp. 2
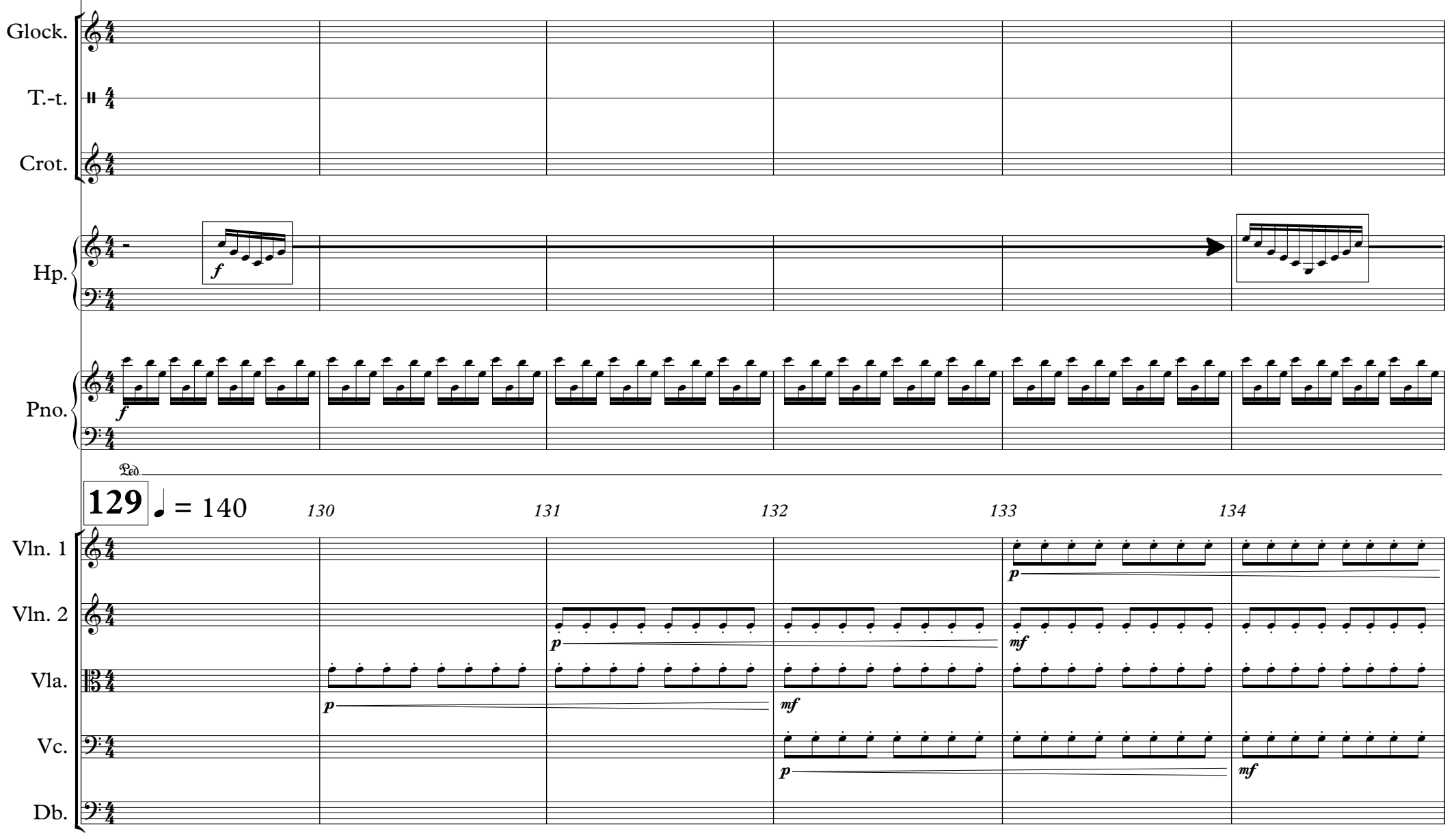

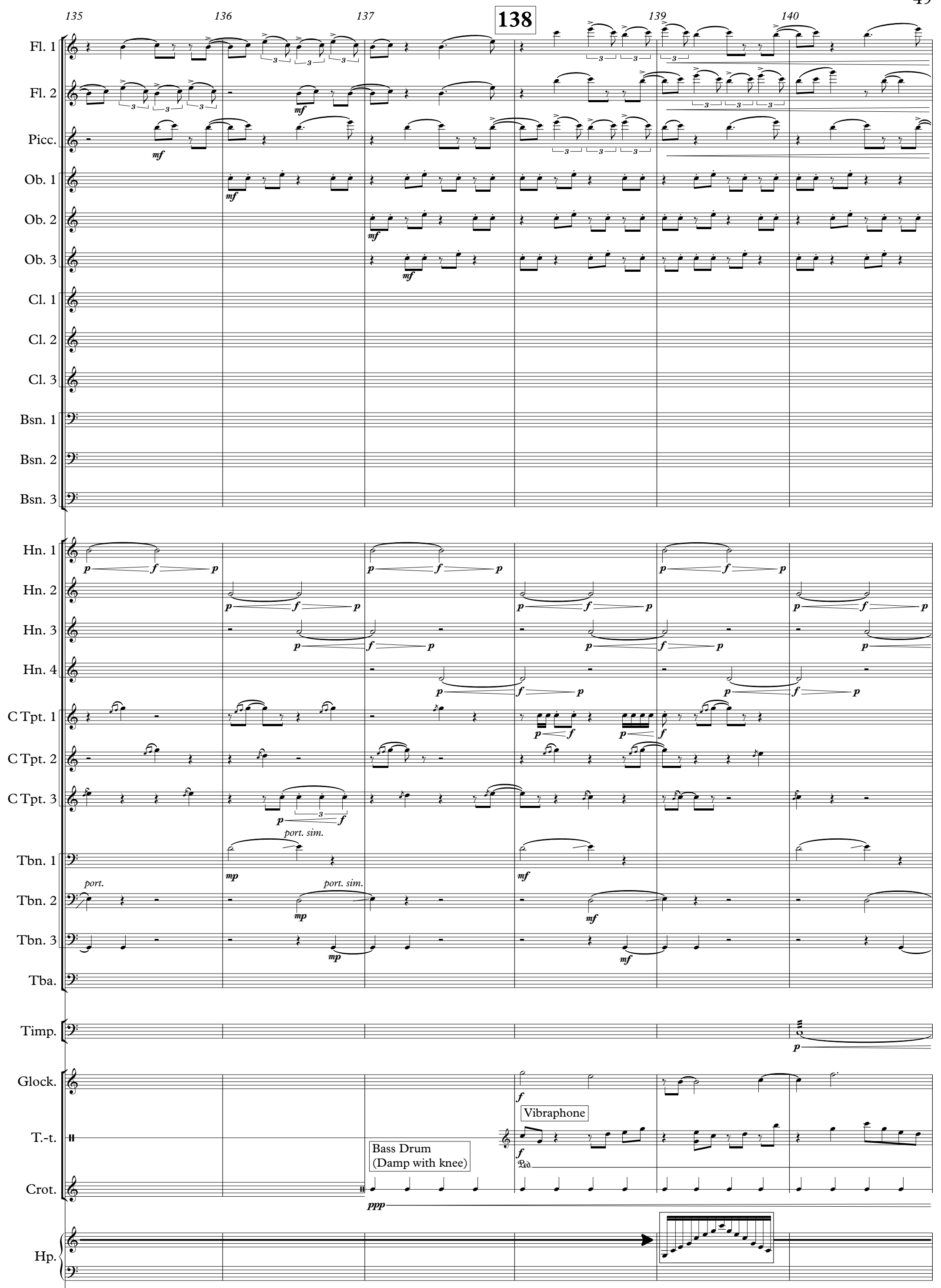
ron

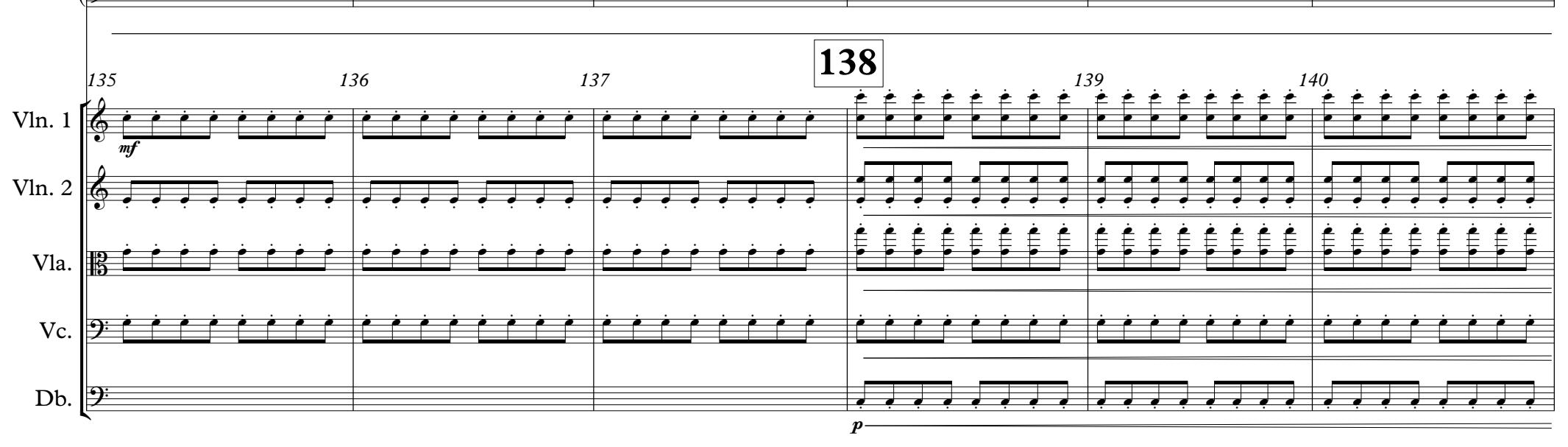



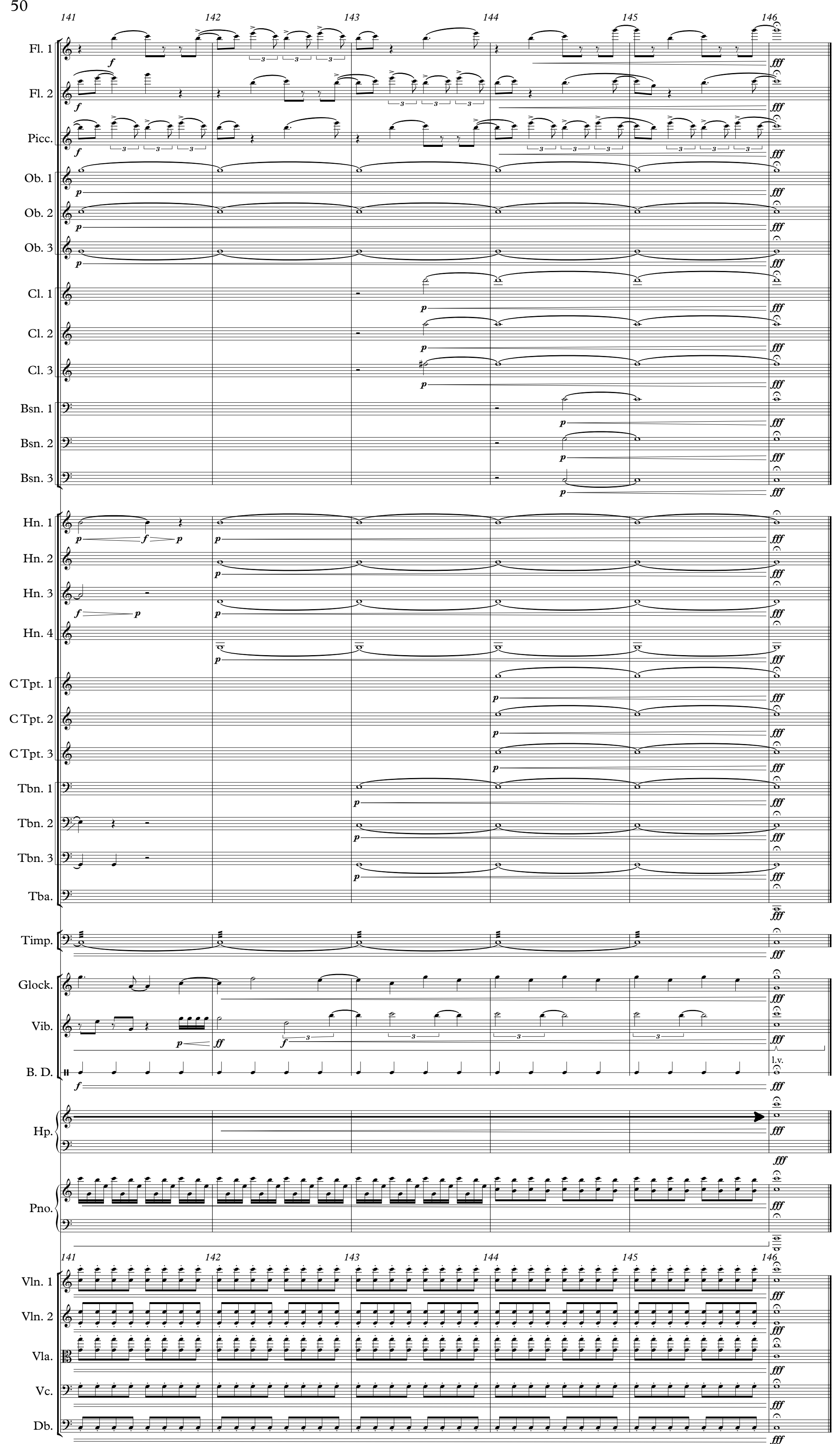


\title{
CURRICULUM VITAE
}

\author{
Lincoln Sandham \\ 4066 Misty Woods Lane, Louisville KY, 40218 \\ lincolnsandham@gmail.com
}

\section{Education}

University of Louisville (UofL), Louisville, KY

Master of Music, Music Composition, 2016-present (degree expected in May 2018)

Baldwin Wallace University (BW), Berea, $\mathrm{OH}$

Bachelor of Music, Music Composition, 2013-2016 - Cum Laude

\section{Composition:}

\section{Primary Instructors}

Dr. Steve Rouse - Coordinator, Composition and Creative Studies, UofL

Dr. Clint Needham - Composer-in-residence, BW

Conducting:

Dr. Jonathan Sokol - Assistant Professor, Composition, BW

Miss Kimcherie Lloyd - Director, Orchestral Studies, UofL Dept. BW

Dr. Dirk Garner - Artistic Director, Bach Festival; Chair, Conducting and Ensembles BW

Dr. Brendan Caldwell - Associate Professor, Conducting; Director, Wind Ensembles, Piano:

Dr. Katherine Kilburn - Composition Performance Coordinator, BW

Dr. Robert Mayerovitch - Chair, Keyboard Dept., BW Voice:

Miss Olga Radosavljevich - Preparatory Piano Dept., Cleveland Institute of Music

Dr. Joanne Uniatowski - Associate Professor, Voice, BW

Miss Marla Berg - Director, Opera Program, Kent State University

\section{Teaching Experience}

2016-present: Theory Lab Coordinator and Teaching Assistant, University of Louisville. Duties include substitute teaching for all sections of Music Theory, administering skills audits, grading, group and one-on-one tutoring, and coordinating and promoting the theory-tutoring lab at UofL. Involves all levels of theory from remedial to advanced post-tonal.

\section{A wards and Honors}

2015-2016 - Elinore Barber Music Scholarship Honoring the Memory of Dr. Albert

\section{Schweitzer}

2013-2016 - BW Transfer Scholar's Award for Academic Talent and Achievement in the

Classroom

2016 - Selected as soloist, BW Honors Recital

2015 - Selected as soloist, BW Convocation Recital

2015 - BW Dean's List Spring

2014 - C7 Prize for Choral Composition (awarded for Falling Slowly in Love for SATB Choir and Piano)

2014 - BW Dean's List Fall

2014 - BW Dean's List Spring

2013 - BW Dean's List Fall

2013 - Outstanding Music Student, Lakeland Community College

Keyboard:

\section{Performance Experience}

2017 - Serve as Harpsichordist for the UofL Early Music Ensemble

2016 - BW Symphonic Wind Ensemble Season and regional tour, including performance at the Kennedy Center in Washington D.C. 


\section{Performance Experience Continued}

Short Ride in a Fast Machine by John Adams - Synthesizer 1

Advance Always by Clint Needham

Concerto No. 2 for Marimba and Wind Ensemble by David Gillingham

2016 - BW Symphony Orchestra Student Composer Concert (rehearsal and premieres of student works)

Prestidigitation by Kevin Thompson

Expectations by Bradley Vogel

A valanche by Jacob Holmes

2016 - BW University Focus Festival, featuring the works of David Lang

Orpheus Over and Under by David Lang- Piano 1

Memory Pieces by David Lang - Soloist

2013-2016 - Served as solo and chamber pianist for the BW Composition Department, rehearsing and performing student compositions.

2015 - BW New Music Ensemble

Paraphrase on Themes of Brian Eno by Timothy Andres

Lullaby by Ned Rorem

Chaconne by John Harbison

Velvet Hammer by Sean Friar

2013 - BW University Focus Festival, featuring the works of Christopher Theofanidis Etenraku by Christopher Theofanidis

Voice:

2017-Present - UofL Cardinal Singers - Tenor 1

2016-2017 - UofL Collegiate Chorale - Tenor 1 - Select performances:

Symphony No. 2 by Gustav Mahler with the Louisville Orchestra

Saint Chapelle by Eric Whitacre

La Petenera by Carlos Sanchez Gutierrez - Soloist

2013-2016 - BW University Choir - Tenor 1 - Select performances:

St. Matthew Passion by J.S. Bach

Mass in B Minor by J.S. Bach

St. John Passion by J.S. Bach

Magnificat in $D$ by J. S. Bach

Wie schön leuchtet der Morgenstern by J.S. Bach

Requiem by Herbert Howells

Messages to Myself by Christopher Theofanidis (BW Focus Festival)

Andrew Norman

Masterclasses

Carlos Sanchez Gutierrez

Transient Canvas

Loris Ohannes Chobanian

David Lang

Christopher Theofanidis

2017:

\section{Works List}

The Gray Raincurtain - Orchestra - 17 minutes

For Bass Trombone and Piano - 7 minutes, Commissioned by Noal McLimore

Sonorous Compass - Small Wind Ensemble - 6 minutes, Commissioned by Paul Vickous

New Work (in progress) - String Quartet - n/a, Commissioned by the Grove City College String Quartet

When You Are Old and Grey - Medium Voice and Piano - 5 minutes 30 seconds

Look Back On Time - SATB Choir - 5 minutes

In Short - Orchestra - 1 minute (for the UofL Orchestra's Fanfare Project)

2016:

Agnus Dei - SATB Choir - 4 minutes 30 seconds

Final - Voice and Electronics - 6 minutes

Alignment - Brass Quintet and Percussion - 5 minutes 30 seconds

The Chair - Electronics (for the Fyoo-zh-en dance festival, BWU) - 4 minutes

Resonant Attacks - Solo Piano - 2 minutes

2015:

Night Sketches - Bass Clarinet and Marimba (for Transient Canvas) - 2 minutes

29 July, 1856 - Chamber Orchestra and Soprano - 6 minutes 
Breathe - Wind Ensemble - 6 minutes

Works List Continued

Suddenly, Light - SATB or SSAA Choir - 3 minutes 30 seconds

Unorthodox Quintet - Clarinet, Saxophone, Piano, and 2 Double Basses - 4 minutes

Mourning - Piano Trio - 12 minutes

Transformations - Orchestra -7 minutes (for the BW Symphony Orchestra)

Fusion - Electronics (for the Fyoo-zh-en dance festival, BWU) - 5 minutes 2014:

Moment for Clarinet, Violin, and Piano - Clarinet, Violin, and Piano - 3 minutes

Falling Slowly in Love - SATB Choir and Piano - 4 minutes

Phases - Flute, 2 Clarinets, Alto Saxophone, Bb Trumpet, Marimba, Harp, Piano, and

Double Bass -5 minutes

Lament - Piano Trio - 6 minutes

2013:

Mallets and Hammers - Piano, Marimba, and Vibraphone - 12 minutes

Forgotten Carousel - Solo Piano - 2 minutes

The Town Wakes Up - Clarinet, Horn, and Piano - 3 minutes

Conversation with a Brook - Solo Piano - 3 minutes

\section{References}

Steve Rouse - Professor of Composition, Composition Department Chair, UofL (502) 314-6515

steve.rouse@louisville.edu

2301 South $3^{\text {rd }}$ St., Louisville, KY, 40208

Rebecca Jemian - Assistant Professor of Music Theory, Coordinator of Music Theory, UofL (502) 852-6997

r.jemian@louisville.edu

2301 South $3^{\text {rd }}$ St., Louisville, KY, 40208

Clint Needham - Composer-in-residence, BW

(440) 826-2397

cdneedha@bw.edu

49 Seminary St, Berea, OH 44017

Jonathan Sokol - Assistant Professor of Composition, BW

(440) 826-2365

jsokol@bw.edu

4562 W. $192^{\text {nd }}$ Street, Cleveland, OH 44135

Dirk Garner - Bach Festival Artistic Director, BW

(440) 826-2384

dgarner@bw.edu

49 Seminary St, Berea, OH 44017

Robert Mayerovitch - Keyboard Department Chair, BW

(440) 826-2371

rmayerov@bw.edu

49 Seminary St, Berea, OH 44017 